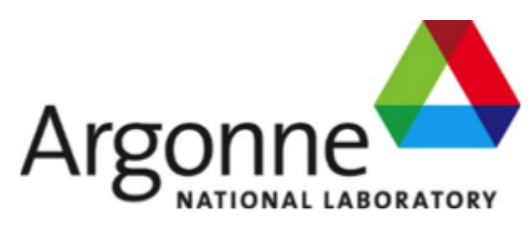

ANL-ART-93

\title{
FY17 Status Report on the Initial Development of a Constitutive Model for Grade 91 Steel
}

Nuclear Engineering Division 
About Argonne National Laboratory

Argonne is a U.S. Department of Energy laboratory managed by UChicago Argonne, LLC under contract DE-AC02-06CH11357. The Laboratory's main facility is outside Chicago, at 9700 South Cass Avenue, Argonne, Illinois 60439. For information about Argonne and its pioneering science and technology programs, see www.anl.gov.

\section{DOCUMENT AVAILABILITY}

Online Access: U.S. Department of Energy (DOE) reports produced after 1991 and a growing number of pre-1991 documents are available free via DOE's SciTech Connect (http://www.osti.gov/scitech/)

Reports not in digital format may be purchased by the public from the National Technical Information Service (NTIS):

U.S. Department of Commerce

National Technical Information Service

5301 Shawnee Rd

Alexandria, VA 22312

www.ntis.gov

Phone: (800) 553-NTIS (6847) or (703)

605-6000 Fax: (703) 605-6900

Email: orders@ntis.gov

Reports not in digital format are available to DOE and DOE contractors from the Office of Scientific and Technical Information (OSTI):

U.S. Department of Energy

Office of Scientific and Technical Information

P.O. Box 62

Oak Ridge, TN 37831-0062

www.osti.gov

Phone: (865) 576-8401

Fax: (865) 576-5728

Email: reports@osti.gov

\section{Disclaimer}

This report was prepared as an account of work sponsored by an agency of the United States Government. Neither the United States Government nor any agency thereof, nor UChicago Argonne, LLC, nor any of their employees or officers, makes any warranty, express or implied, or assumes any legal liability or responsibility for the accuracy, completeness, or usefulness of any information, apparatus, product, or process disclosed, or represents that its use would not infringe privately owned rights. Reference herein to any specific commercial product, process, or service by trade name, trademark, manufacturer, or otherwise, does not necessarily constitute or imply its endorsement, recommendation, or favoring by the United States Government or any agency thereof. The views and opinions of document authors expressed herein do not necessarily state or reflect those of the United States Government or any agency thereof, Argonne National Laboratory, or UChicago Argonne, LLC. 


\section{FY17 Status Report on the Initial Development of a Constitutive Model for Grade 91 Steel}

Nuclear Engineering Division

Argonne National Laboratory

July 2017

Prepared by

M. C. Messner, Argonne National Laboratory

V.-T. Phan, Argonne National Laboratory

T.-L. Sham, Argonne National Laboratory 



\section{Abstract}

Grade 91 is a candidate structural material for high temperature advanced reactor applications. Existing ASME Section III, Subsection HB, Subpart B simplified design rules based on elastic analysis are setup as conservative screening tools with the intent to supplement these screening rules with full inelastic analysis when required. The Code provides general guidelines for suitable inelastic models but does not provide constitutive model implementations. This report describes the development of an inelastic constitutive model for Gr. 91 steel aimed at fulfilling the ASME Code requirements and being included into a new Section III Code appendix, HBB-Z. A large database of over 300 experiments on Gr. 91 was collected and converted to a standard XML form. Five families of Gr. 91 material models were identified in the literature. Of these five, two are potentially suitable for use in the ASME code. These two models were implemented and evaluated against the experimental database. Both models have deficiencies so the report develops a framework for developing and calibrating an improved model. This required creating a new modeling method for representing changes in material rate sensitivity across the full ASME allowable temperature range for Gr. 91 structural components: room temperature to $650^{\circ} \mathrm{C}$. On top of this framework for rate sensitivity the report describes calibrating a model for work hardening and softening in the material using genetic algorithm optimization. Future work will focus on improving this trial model by including tension/compression asymmetry observed in experiments and necessary to capture material ratcheting under zero mean stress and by improving the optimization and analysis framework. 



\section{Table of Contents}

Abstract i i

Table of Contents $\quad$ iii

List of Figures $\quad$ v

List of Tables $\quad$ ix

1 Introduction 1

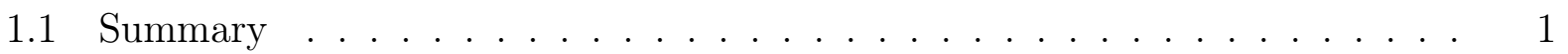

1.2 Gr. 91 microstructure . . . . . . . . . . . . . . . . . . . . . . . . . . . . . .

1.3 Deformation mechanisms . . . . . . . . . . . . . . . 3

2 Experimental Data $\quad 5$

2.1 Thermomechanical conditions . . . . . . . . . . . . . . . 5

2.2 Sources and summary of experimental data . . . . . . . . . . . . 5

2.2.1 Yield stress and work hardening under monotonic loading . . . . . . . 6

2.2.2 Cyclic experiments . . . . . . . . . . . . . . . . . . 10

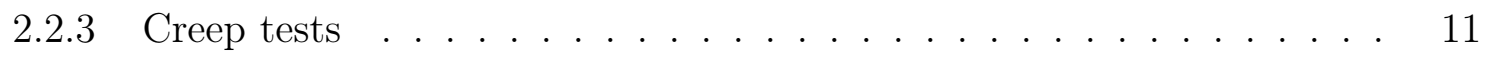

2.3 Rate sensitivity . . . . . . . . . . . . . . . . . . . . . . . . . . . . . . . . . . . .

2.3.1 In tensile experiments . . . . . . . . . . . . . . . . . 14

2.3.2 In creep experiments . . . . . . . . . . . . . . . . . 16

2.3.3 Combined model . . . . . . . . . . . . . . . . . . . . . 17

3 Viscoplastic material models 21

3.1 General framework . . . . . . . . . . . . . . . . . . . . . 21

3.2 Gr. 91 material models in the literature . . . . . . . . . . . . . . . . 22

3.3 Model evaluation . . . . . . . . . . . . . . . . . . . 24

4 Implementation and performance of the Koo and Yaguchi models 25

4.1 Koo \& Kwon model . . . . . . . . . . . . . . . . . . . . . . . . . 27

4.2 Yaguchi \& Takahashi model . . . . . . . . . . . . . . . . . . . . 32

4.3 Model performance . . . . . . . . . . . . . . . . . . 34

5 Modeling the rate sensitivity of advanced reactor structural materials 39

5.1 Introduction . . . . . . . . . . . . . . . . . . 39

5.2 Modeling the transition between rate sensitive and rate insensitive response . $\quad 40$

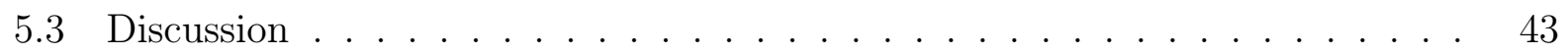

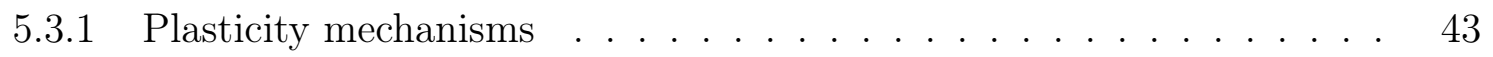

5.3 .2 Work hardening . . . . . . . . . . . . . . . . . . 44

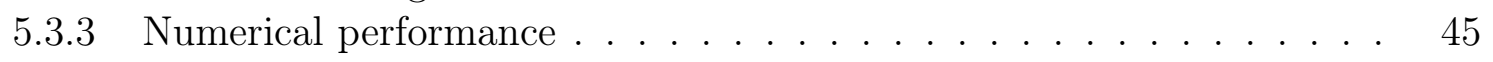

5.4 Conclusions . . . . . . . . . . . . . . . . . . . . 46

6 Preliminary ANL constitutive model 49

6.1 Fitting a model with genetic algorithm optimization . . . . . . . . . . . . 49

6.2 Improved calibration strategy . . . . . . . . . . . . . . . . 56

6.3 Conclusions and ongoing work . . . . . . . . . . . . . . 56

7 Conclusions and future work 61

7.1 Experimental data and existing models . . . . . . . . . . . . . . 61

7.2 New constitutive models . . . . . . . . . . . . . . . . . . . . 61

A XML Data Format $\quad 63$

ANL-ART-93 iii 
FY17 Status Report on the Initial Development of a Constitutive Model for Grade 91 Steel July 2017

$\begin{array}{ll}\text { Acknowledgments } & 67\end{array}$

$\begin{array}{ll}\text { Bibliography } & 69\end{array}$

Distribution list $\quad 75$ 


\section{List of Figures}

1.1 Sketch of a typical Gr. 91 prior austenite grain. Only the PAG and packets are approximately equiaxed. The block and lath structure is laminar and aligned, based on a common martensite to austenite transformation. . . . . . 2

2.1 Plot summarizing the yield stress data obtained from monotonic tension tests. 7

2.2 Two typical uniaxial tension test stress/strain curves. At lower temperatures Gr. 91 exhibits work hardening, at higher temperatures work softening behavior. Plots show true stress and strain. . . . . . . . . . . . . . 8

2.3 Empirical Voce + linear isotropic hardening/softening scheme. . . . . . . . 8

2.4 Plots of the hardening parameters $\sigma_{\max }$ and $\theta_{0}$ normalized by the temperature dependent shear modulus $\mu$ versus normalized activation energy $g$. Plots are generated using true stress and true strain data. . . . . . . . . . . .

2.5 Plot of the terminal softening slope $\theta_{\text {soften }} / \mu$ versus normalized activation energy. . . . . . . . . . . . . . . . . . . 10

2.6 Saturated decay stress, normalized by the temperature-dependent shear modulus, versus normalized activation energy. . . . . . . . . . . . .

2.7 Results from a variety of stress controlled cyclic tests. a) Effect of load ratio $R, \mathrm{~b})$ effect of loading rate $\dot{\sigma} \ldots \ldots \ldots \ldots$

2.8 Kocks-Mecking plots of the minimum creep rate (a) and initial primary creep rate (b) from the experiments.

2.9 Temperature-dependent shear modulus model compared to shear modulus values for Gr. 91 calculated from the ASME BPVC Section II, Part D, Table TM values of Young's modulus and the Section II temperature-independent Poisson's ratio.

2.10 Kocks-Mecking, Arrhenius-type analysis of the a) yield and b) flow stress at $2.5 \%$ strain of Gr. 91, using all the identified monotonic data. The graphs plot the stress, normalized by the temperature-dependent shear modulus of the material versus the normalized activation energy. Both plots indicate a transition from rate insensitive deformation to rate sensitive deformation at a normalized activation energy of about $g=0.4$. Both plots use the same reference strain rate $\dot{\varepsilon}_{0}=10^{12} \mathrm{~s}^{-1}$.

2.11 Kocks-Mecking plot of the minimum creep rates from 96 experiments. . . . 17

2.12 Kocks-Mecking plot combining data from the uniaxial tension and creep experiments.

2.13 Plots show the regions of rate sensitivity for Gr. 91 in terms of temperature and strain rate. a) shows a full temperature range and b) zooms into the temperature range where the corresponding strain rates are experimentally accessible.

2.14 Plot of temperature dependent rate sensitivity exponent $m$ implied by the Kocks-Mecking model with $a=6.5$. 
4.1 Verification of the implementation of the Koo \& Kwon model. Plots compare results from the implemented model (black lines) for strain controlled cyclic deformation at $\dot{\varepsilon}=10^{-4} \mathrm{~s}^{-1}$ in the strain range $\pm 0.6 \%$ to results from Koo and Kwon [41] (dashed lines, filled region) at a) $500^{\circ} \mathrm{C}$, b) $550^{\circ} \mathrm{C}$, and c) $600^{\circ} \mathrm{C}$.

4.2 Comparison of Koo \& Kwon model to uniaxial tension experimental data: a) yield stresses and b) initial hardening slope. . . . . . . . . . . . . . 30

4.3 Comparison of Koo \& Kwon model to strain controlled cyclic softening tests.

4.4 Comparison between Koo \& Kwon model and experiments for stress-controlled cyclic loading. a) Effect of load ratio $R$, b) effect of loading rate $\dot{\sigma}$, and c) effect of hold time (hold at maximum tensile stress). . . . . . . . . . . .

4.5 Kocks-Mecking plots of the minimum creep rate (a) and primary creep rate (b) comparing the Koo \& Kwon model to the experiments. . . . . . . . . .

4.6 Plots verifying the implementation of the Yaguchi \& Takahashi model. a) Simulations of uniaxial tension tests at various strain rates. b) Simulations of stress relaxation tests, with initial loading performed at various strain rates. The current implementation exactly matches the results provided in [66] and so the curves fall directly on top of each other. . . . . . . . . . . . . .

4.7 Comparison of Yaguchi \& Takahashi model to uniaxial tension experimental data: a) yield stresses and b) initial hardening slope. . . . . . . . . . . . .

4.8 Comparison of Yaguchi \& Takahashi model to strain controlled cyclic softening tests.

4.9 Comparison between Yaguchi \& Takahashi model and experiments for stresscontrolled cyclic loading. a) Effect of load ratio $R$, b) effect of loading rate $\dot{\sigma}$, c) effect of hold time (hold at max tensile stress). . . . . . . . . . . . .

4.10 Kocks-Mecking plots of the minimum creep rate (a) and primary creep rate (b) comparing the Yaguchi \& Takahashi model to the experiments. . . . . .

5.1 Rate sensitivity diagrams for Grade 91 steel. (a) Kocks-Mecking plot summarizing experimental data and establishing bilinear fit. (b) 3D plot illustrating the dependence of normalized flow stress on temperature and strain rate with the transition between rate sensitive and insensitive behavior labeled. . . . .

5.2 This plot compares a series of simulated tests using the model described here for Gr. 91 to the direct bilinear fit to the experimental data. The rate sensitivity of the composite model causes all the simulated test data, for monotonic tension, creep, and stress relaxation tests, to fall directly on the best fit line describing the experimental data. . . . . . . . . . . . .

5.3 Simulation using the calibrated strain rate sensitivity model at $750^{\circ} \mathrm{C}$ and Voce isotropic hardening. In the figure the black line plots the flow stress $\sigma_{f}$ and the red line plots the difference $\sigma_{f}-\sigma_{y}$ with $\sigma_{y}$ the yield stress at the current strain rate. This simulation demonstrates the continuation of work hardening across the rate sensitive/insensitive boundary and the ability of the model to transition from rate sensitive to rate insensitive behavior and subsequently back to rate sensitive deformation. The labeled strain rates have units of $s^{-1}$. 
5.4 Example simulation of a load history involving rapid strain rate oscillations. (a) The strain rate history imposed on the model. (b) The resulting stress/strain curve. . . . . . . . . . . . . . . . . . . . . . . . . .

6.1 Plot illustrating the convergence of the genetic algorithm optimization method. 52

6.2 Plot comparing the GA model to experimental data, the Koo \& Kwon model, and the Yaguchi model for uniaxial tension loading at various strain rates. Subfigure a) shows results for $T=550^{\circ} \mathrm{C}$ and b) shows results for $T=600^{\circ} \mathrm{C}$.

6.3 Plot comparing the GA model to experimental data, the Koo \& Kwon model, and the Yaguchi model for creep loading at various stresses. Subfigure a) shows results for $T=550^{\circ} \mathrm{C}$ and b) shows results for $T=600^{\circ} \mathrm{C}$. . . . .

6.4 Plot comparing the GA model to experimental data, the Koo \& Kwon model, and the Yaguchi model for stress relaxation at at various initial strains. Subfigure a) shows results for $T=550^{\circ} \mathrm{C}$ and b) shows results for $T=600^{\circ} \mathrm{C}$. .

6.5 Plot comparing the GA model to experimental data, the Koo \& Kwon model, and the Yaguchi model for stress controlled cyclic loading at various stress ratios at $T=550^{\circ} \mathrm{C} \ldots \ldots \ldots \ldots \ldots \ldots \ldots$

6.6 Plot comparing the GA model to experimental data, the Koo \& Kwon model, and the Yaguchi model for fully-reversed stress controlled cyclic loading with various hold times at peak load at $T=550^{\circ} \mathrm{C} \ldots \ldots \ldots \ldots$

6.7 Plot comparing the GA model to experimental data, the Koo \& Kwon model, and the Yaguchi model for fully-reversed stress controlled cyclic loading with various stress rates at $T=550^{\circ} \mathrm{C} \ldots \ldots \ldots \ldots \ldots$

6.8 Plot comparing the manually calibrated model to experimental data, the Koo \& Kwon model, and the Yaguchi model for uniaxial tension loading at various strain rates at $T=550^{\circ} \mathrm{C} \ldots \ldots \ldots \ldots \ldots$

6.9 Plot comparing the manually calibrated model to experimental data, the Koo \& Kwon model, and the Yaguchi model for creep loading at various stresses at $T=550^{\circ}$ C . . . . . . . . . . . . . . . . . .

6.10 Plot comparing the manually calibrated model to experimental data, the Koo \& Kwon model, and the Yaguchi model for stress relaxation at at various initial strains at $T=550^{\circ} \mathrm{C} \ldots \ldots \ldots \ldots$

6.11 Plot comparing the manually calibrated model to experimental data, the Koo \& Kwon model, and the Yaguchi model for stress controlled cyclic loading at various stress ratios at $T=550^{\circ} \mathrm{C} \ldots \ldots \ldots \ldots \ldots$

6.12 Plot comparing the manually calibrated model to experimental data, the Koo \& Kwon model, and the Yaguchi model for fully-reversed stress controlled cyclic loading with various hold times at peak load at $T=550^{\circ} \mathrm{C} . \quad \ldots$.

6.13 Plot comparing the manually calibrated model to experimental data, the Koo \& Kwon model, and the Yaguchi model for fully-reversed stress controlled cyclic loading with various stress rates at $T=550^{\circ} \mathrm{C} \ldots \ldots \ldots \ldots$ 



\section{List of Tables}

2.1 Overview of the experimental data included in the database. . . . . . . . 6

3.1 Summary of constitutive models for Gr. 91 identified in the literature. The temperature variation column describes how the model scales its parameters with changes in the temperature. . . . . . . . . . . . . . .

4.1 Material parameters provided in Koo and Kwon [41] for Gr. 91. Poisson's ratio was not given in the work as the implementation was uniaxial. The

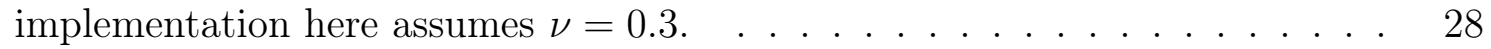

5.1 Bilinear descriptions of the master curve for Gr. 91. . . . . . . . . . . . 41

6.1 Optimization hyperparameters used in this subsection. . . . . . . . . 51 



\section{Introduction}

\subsection{Summary}

ASME Section III Division 5, Subsection HB, Subpart B provides simplified design rules based on elastic analysis results to satisfy deformation limits for Class A components in elevated temperature service. These bounding methods were established as conservative screening tools and the expectation was that inelastic analyses would sometimes be required. The simplified design methods were developed based on the assumption of uncoupled plasticity and creep. When such conditions are not met, the newly developed elastic, perfectly plastic (EPP) evaluation methods could be used as alternative screening tools. Alternatively, designers could also use full inelastic analysis.

Rules are provided in Subsection HB, Subpart B to satisfy deformation limits for Class A materials using inelastic analysis results. While specific constitutive models for Class A materials are not provided in Division 5, paragraph HBB-3214.2 describes the following key features that are required in the constitutive equations:

- effects of temperature-dependent plastic hardening or softening, including cyclic loading effects;

- primary creep and the effects of creep strain hardening and softening due to reverse loadings;

- effect of prior creep on subsequent plasticity and vice versa.

Since constitutive equations are not provided in Division 5, designers would need to document and justify in the Design Report the model employed to generate inelastic analysis results for design evaluations per Division 5 requirements on deformation limits for structural integrity. In order to address this gap, an ASME code committee was formed to develop constitutive equations for Class A materials and to establish guidance on inelastic analysis methods for incorporation in a new appendix, HBB-Z, of Division 5, Subsection HB, Subpart B. This work develops a constitutive model for one of the Class A materials, 9Cr-1Mo-V, or Gr. 91 steel, in support of Division 5 Appendix HBB-Z development.

This report describes and collects the data required to calibrate a full inelastic constitutive description of Gr. 91, evaluates the performance of existing constitutive models, and describes the initial development of a new, improved model for Gr. 91 that spans the full expected temperature and strain rate conditions reactor structures will see in service. The report identifies the key deformation mechanisms a successful model must capture and relates these mechanisms to the microstructure of Gr. 91. The experimental database assembled here collates the results of over 300 experiments on Gr. 91 material suitable for model calibration. Five families of Gr. 91 material models in the literature are identified. From these five the report describes the implementation of two of the most promising and compares the response of these two models to the experimental database. This comparison shows both models fail to adequately capture the strain rate sensitivity of Gr. 91 across the full expected temperature range and so a new method for representing material rate sensitivity is developed and calibrated directly against the experimental database. Finally, the report describes adding models for work hardening and softening in Gr. 91 to the model for rate sensitivity to 


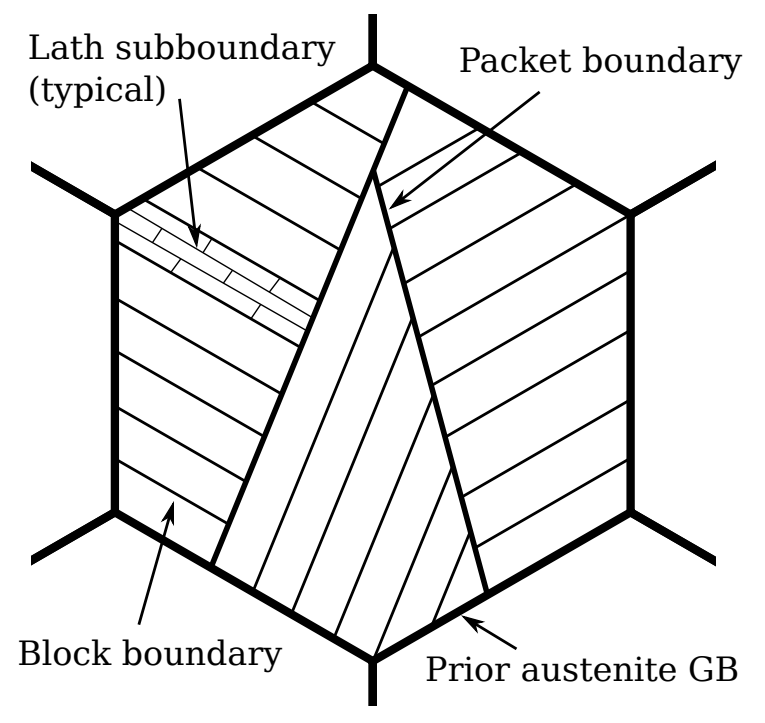

Figure 1.1: Sketch of a typical Gr. 91 prior austenite grain. Only the PAG and packets are approximately equiaxed. The block and lath structure is laminar and aligned, based on a common martensite to austenite transformation.

develop a complete constitutive description of the material. Ultimately, this material model will be calibrated with genetic optimization methods to the complete experimental database. However, these algorithms only fit coefficients; they do not determine the form of the model. Furthermore, this report demonstrates genetic algorithms need a good starting population of models and good bounds on the model parameters to produce a good final model. So the report describes the manual calibration of a viscoplastic model for Gr. 91 at $550^{\circ} \mathrm{C}$. This model, with some possible future improvements to capture tension/compression asymmetry observed in Gr. 91, determines the form of the model for the genetic optimization and will provide the optimization scheme with a good starting candidate to further optimize against the full data set. Finally, the report summarizes the accomplishments of the project over this fiscal year and describes future work required to develop a final model for Gr. 91.

\subsection{Gr. 91 microstructure}

Gr. 91 has a complex microstructure. On the coarsest scale the microstructure of Gr. 91, and tempered martensite steels in general, consists of collections martensite laths arrange inside larger prior austenite grains (PAGs) [5, 30]. The laths inside a PAG can be grouped into blocks which all followed the same austenite to martensite transformation and packets of roughly-parallel, aligned blocks. Subgrain boundaries developed during tempering separate laths within a block [28]. These subgrains can be viewed as a consequence of the dense dislocation structure. The mean PAG size is $30 \mu \mathrm{m}$ and the mean lath size is $0.2 \mu \mathrm{m}$ [28]. There are generally several packets per PAG, with packet sizes around $30 \mu \mathrm{m}$ and individual blocks have mean width of $1 \mu \mathrm{m}$. Figure 1.1 schematically illustrates the PAG substructure.

Initial dislocation densities in the laths are the on order of $10^{13} \mathrm{~m}^{-2}$, arranged in a dense, planar honeycomb structure [30]. Dispersed in the substructure are a variety of carbide 
and nitride precipitates, primarily located along lath and prior austenite grain boundaries $[5,30]$. The dispersion of these fine precipitates is thought to play a critical role in developing increased creep strength at elevated temperatures [2, 31]. Precipitate sizes are on the order of $100 \mathrm{~nm}$ [58]. Additionally, after tempering, $\alpha$-ferrite matrix forms along lath and PAG boundaries [30].

This PAG substructure substantially coarsens during plastic deformation. The dense lath dislocation structure relaxes considerably, leading to a coarsening of the subgrains [30, 18]. Choudhary and Isaac Samuel [18] observe that, at the end of a long period of creep deformation, even PAG boundaries become indistinct, indicating a substantial rearrangement of the microstructure. Additionally, as creep deformation progresses the precipitates also coarsen, increasing in size [18]. These changes to the substructure suggest plausible mechanisms for plastic deformation.

\subsection{Deformation mechanisms}

The usual mechanism of plastic deformation in ferritic/martensitic steels at moderate strain rates (around the range $10^{-6} \mathrm{~s}^{-1}$ to $10^{-2} \mathrm{~s}^{-1}$ ) and temperatures (above room temperature but below the recrystallization temperature) is the glide of dislocations between microstructural obstacles and various mechanism these glissile dislocations use to overcome the energy barrier presented by an obstacle [40]. These obstacles may be fine precipitates, solute atoms, or, commonly, other sessile dislocations. The mechanism of dislocation glides has only moderate temperature dependence and, in this temperature and strain rate regime, occurs much faster than the processes a dislocation uses to overcome an obstacle [40]. Therefore, the kinetics of overcoming these obstacles governs the deformation of the material.

When a glissile dislocation glides into a sessile dislocation the stress field of the two can interact to oppose continued motion. This interaction forms a jog, increasing the sessile dislocation density in the grain and increasing the force required to subsequently move glissile dislocations through the dislocation structure. Similar pinning mechanisms can be observed for precipitates and solution strengthening mechanisms. These pinned dislocations are the simplest explanation of work hardening in conventional metal alloys.

However, several mechanisms can unpin such a dislocation, leading to continued glide. One important mechanism in ferritic/martensitic steels is edge dislocation climb. Here an edge dislocation, assisted by atomistic diffusion, moves perpendicular to its glide plane. Often this mechanism can cause two dislocation segments with opposite burgers vectors to meet, leading to their annihilation, a decrease in the overall dislocation density, and an overall reduction in the work hardening rate. In Gr. 91 the initial dislocation structure is so dense that relaxation leads to work softening. Climb is a thermally-activated mechanism, requiring some free kinetic energy to carry out the diffusion-aided process. This free energy can be provided by the thermal vibration of the lattice atoms, measured by the absolute temperature of the system, or the kinetic energy of the dislocation itself, represented by the system strain rate. Again, because dislocation glide occurs much quicker than climb it is the kinetics of the climb process that governs the deformation of the material.

At higher stresses, near to the yield stress, and lower temperatures creep in Gr. 91 steels essentially occurs through this same mechanism: a combination of dislocation glide and climb with the kinetics of climb controlling the deformation rate [58]. At lower stresses a purely 
diffusion governed mechanism develops instead [58]. There is some debate in the literature as to the exact form of this mechanism, any of Coble creep [20], Harper-Dorn creep [32], and Herring-Nabarro creep are plausible [33]. An additional plausible mechanism for softening at low stresses is the diffusion of solute atoms to the precipitates, resulting in precipitate coarsening and a reduction in solution hardening.

As with the glide/climb mechanism all these plausible diffusional mechanisms are thermallyactivated. Therefore, the deformation of Gr. 91 at both high and low stresses is controlled by the availability of free kinetic energy in the material structure. Furthermore, at moderate temperatures there is essentially no difference between the mechanisms of creep and plastic deformation - they are both governed by the climb of dislocations. Therefore, the two mechanisms are inextricably linked. The next chapter applies this concept of thermal activation to describe a large collection of experimental results measuring the material flow stress in a wide range of experimental conditions. 


\section{Experimental Data}

\subsection{Thermomechanical conditions}

The maximum use temperature for Gr. 91 in ASME Division 5, Subsection HB, Subpart B is $650^{\circ} \mathrm{C}$. Hence a constitutive model for Gr. 91 in support of the development of the new Division 5 Appendix HBB-Z on inelastic analysis methods for Class A materials is required to represent the material response ranging from room temperature to the maximum use temperature in the Code.

At elevated temperatures creep and cyclic plasticity are not independent deformation mechanisms [16, 44]. A successful model must capture creep-fatigue interaction in the service temperature range as accurately as possible. The best source of experimental data are then cyclic tests with stress or strain holds during the cycle, allowing for creep or stress relaxation to occur in concert with cyclic fatigue. While many experiments of this type are reported in the literature $[24,26,25,27,30,67,35]$, only a few reports provide useful data for calibrating a material model, i.e. details of the cyclic stress/strain response rather than simply failure life data. Second best are stress or strain controlled cyclic experiments without hold times and creep and stress relaxation experiments without cycling. There is a large amount of data available of these types. The cyclic experiments can constrain the kinematic hardening and cyclic softening properties of a material model while the creep and stress relaxation experiments describe the material rate dependence.

Uniaxial tension tests at service conditions and rates are useful for calibrating model rate sensitivity and base flow stress. Additional uniaxial tension data at various temperatures and strain rates can help describe the material rate sensitivity. Data from creep experiments is required to determine tertiary creep response.

Finally, several experiments should be recognized that are not included in the experimental database detailed in the next subsection. Fournier et al. [24, 26, 25] conducted an extensive series of combined creep/fatigue experiments. However, there data is presented in terms of accumulated creep/fatigue damage and cycles to failure and so is of limited help in fitting a model. However, these tests serve as a good source of data for the final validation of a calibrated model. Several other works describe experimental data in a similar format or in some way otherwise incompatible with the standard formats adopted here and so are not included in the database: [19, 30, 59]. In addition to more standard experiments, Yaguchi and Takahashi [67] conducted a series of innovative multiaxial fatigue tests. The database includes the data from their more conventional tests but excludes the multiaxial tests as it is difficult to succinctly summarize the loading conditions in such as way as to allow quickly simulating the tests with a prospective material model. Lastly, data provided in Kloc and Sklenička [37] was excluded from the fit database because all the data collected from that paper are extreme outliers. The cause of this discrepancy is currently unknown - it may reflect differences in processing and forming conditions.

\subsection{Sources and summary of experimental data}

Given the criteria described in the previous subsection, Table 2.1 lists the sources of experimental data identified in the literature. The table cites the work and summarizes the types of data the source provides. 
FY17 Status Report on the Initial Development of a Constitutive Model for Grade 91 Steel

July 2017

\begin{tabular}{ll}
\hline Source & Data provided \\
\hline Choudhary and Isaac Samuel [18] & Creep tests \\
Kim and Weertman [35] & Strain controlled fatigue tests, including some with holds \\
Kimura et al. [36] & Creep tests \\
Koo and Lee [42] and Koo and Kwon [41] & Strain controlled fatigue and stress relaxation \\
Latha et al. [45] & Tension and creep tests \\
Personal communication with M. Li & Tension tests \& strain controlled fatigue tests, \\
of Argonne National Laboratory & including holds \\
Maruyama et al. [47] & Long term creep tests \\
Swindeman [61] and Swindeman [62] & Tension and creep tests \\
Yaguchi and Takahashi [65] & Tension and stress relaxation tests \\
Yaguchi and Takahashi [67] & Tension and stress and strain controlled fatigue tests, \\
& including some with holds \\
\hline
\end{tabular}

Table 2.1: Overview of the experimental data included in the database.

Data from all these sources was collated into a standard XML format described in Appendix A. All together the database contains 329 individual experiments spanning a large range of temperatures and strain rates.

The large quantity of information in the database makes fitting or comparing models to the data difficult. Each experiment represents a time series of stress/strain data under a some defined loading and environmental conditions. A complete comparison with a model would then plot both the experiment's stress/strain series and the corresponding stress/strain history generated from the model under the same loading and environmental conditions. Repeating this process for each experiment would generate several hundred plots, making a full comparison difficult. Additionally, for cyclic experiments a stress/strain plot can obscure features of interest. Typically plots summarizing the results of cyclic experiments plot one or multiple of the maximum, minimum, and mean stress or strain (depending on how the load was applied) versus the cycle number. These plots better illustrate the development of cyclic hardening or softening and the possible evolution of ratcheting strain.

Data reduction is then a critical task. Ideally, the full data set would reduce to a limited number of plots summarizes critical features over a large range of experimental conditions. Comparing a model to the experiment data still requires simulating a large number of full stress/strain histories, but at least the results can be compactly summarized. The next few sections describes various methods of collecting multiple experiments, or critical features of experiments, into a single plot summarizing the data.

\subsubsection{Yield stress and work hardening under monotonic loading}

The Kocks-Mecking approach [40, 38, 39, 48] to determining the rate dependence of a material is described in more detail in Section 2.3. Here their idea of a normalized activation energy combining temperature and strain rate effects

$$
g=\frac{k T}{\mu b^{3}} \log \frac{\dot{\varepsilon}_{0}}{\dot{\varepsilon}}
$$




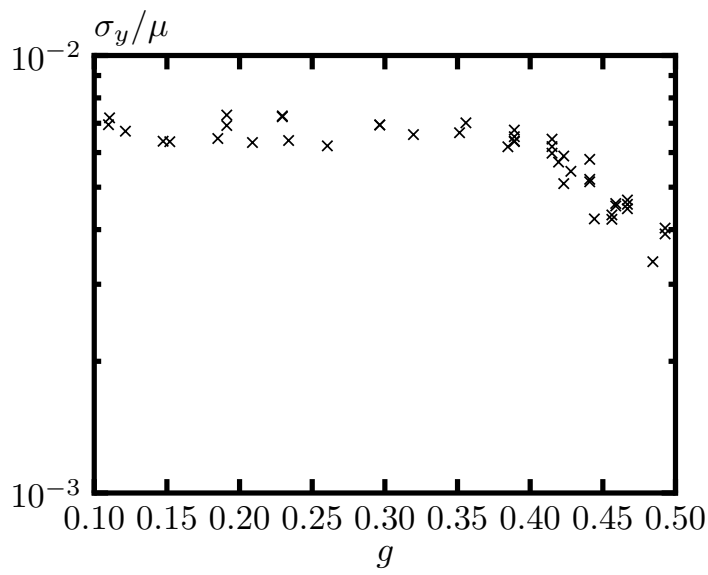

Figure 2.1: Plot summarizing the yield stress data obtained from monotonic tension tests.

with $k$ the Boltzmann constant, $T$ the absolute temperature, $\mu$ the temperature dependent shear modulus, $b$ a relevant Burgers vector, $\dot{\varepsilon}_{0}$ a reference strain rate, and $\dot{\varepsilon}$ a characteristic strain rate describing the material deformation. Here log is the natural logarithm. Section 2.3 also describes the temperature-dependent shear modulus and the reference strain rate used to calculate the normalized activation energies for Gr. 91.

Figure 2.1 plots the yield stress determined in monotonic tension tests, normalized by the shear modulus, versus the normalized activation energy. The data collapses to a linear relation within two energy ranges, reflecting a change from a rate-independent deformation mechanisms to a rate dependent mechanism at higher temperatures.

Figure 2.2 plots the stress/strain data from two monotonic tension tests - one at room temperature $\left(20^{\circ} \mathrm{C}\right)$ and one at high temperature $\left(600^{\circ} \mathrm{C}\right)$ [61]. These two curves are typical for the two temperature regimes. Gr. 91 exhibits work hardening behavior at lower temperatures but then transitions to work softening behavior at higher temperatures [35]. Microstructural characterization during high temperature $\left(550^{\circ} \mathrm{C}\right)$ indicates recrystallization and dynamic recovery from initially high dislocation densities cause the observed work softening [35]. Studies on other ferritic-martensitic steels indicate the interplay between recovery mechanisms affecting the dislocation structure without strong recrystallization and full recrystallization depends on the initial amount of cold work, and hence the initial dislocation density [3].

Both mechanisms are thermally activated and so the rate of work softening should depend on the Kocks-Mecking normalized activation energy. However, traditional models assume the rate of work hardening via Stage II processes is athermal [56]. Proceeding from these assumptions and assuming first order recovery and the usual Taylor relation between dislocation density and flow stress yields a Voce model [55, 39]. Such a model predicts an increase or decrease in flow stress from the initial value, saturating at some constant level. The usual Voce model cannot predict an initial increase in flow stress (work hardening) followed by a peak value and then a decrease. Several flow curves in the dataset show this behavior. 
FY17 Status Report on the Initial Development of a Constitutive Model for Grade 91 Steel July 2017

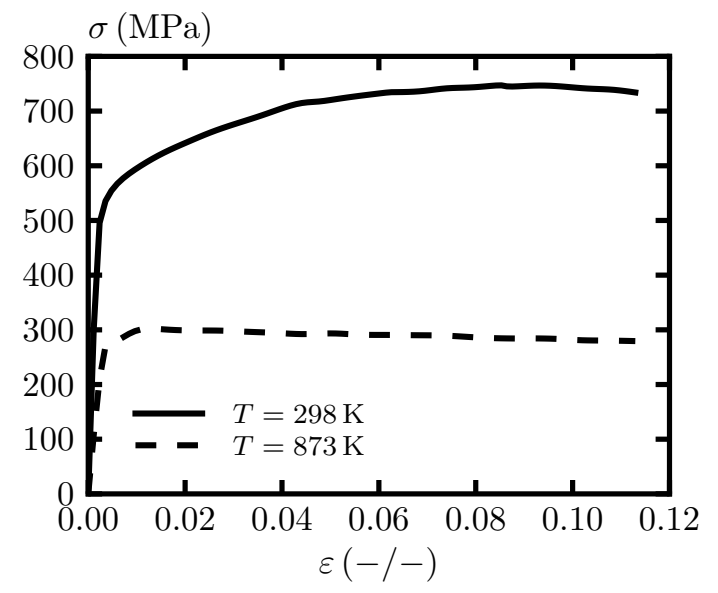

Figure 2.2: Two typical uniaxial tension test stress/strain curves. At lower temperatures Gr. 91 exhibits work hardening, at higher temperatures work softening behavior. Plots show true stress and strain.

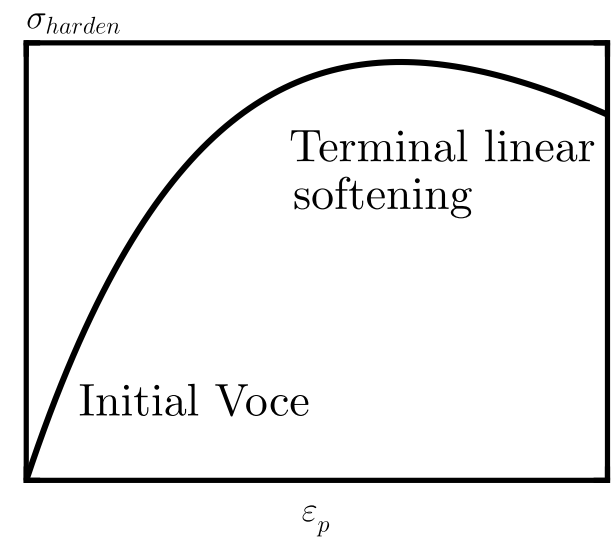

Figure 2.3: Empirical Voce + linear isotropic hardening/softening scheme. 


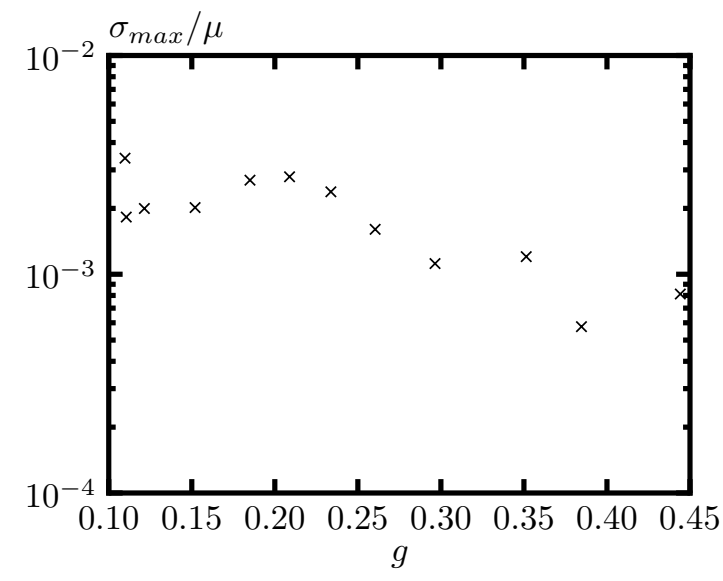

a)

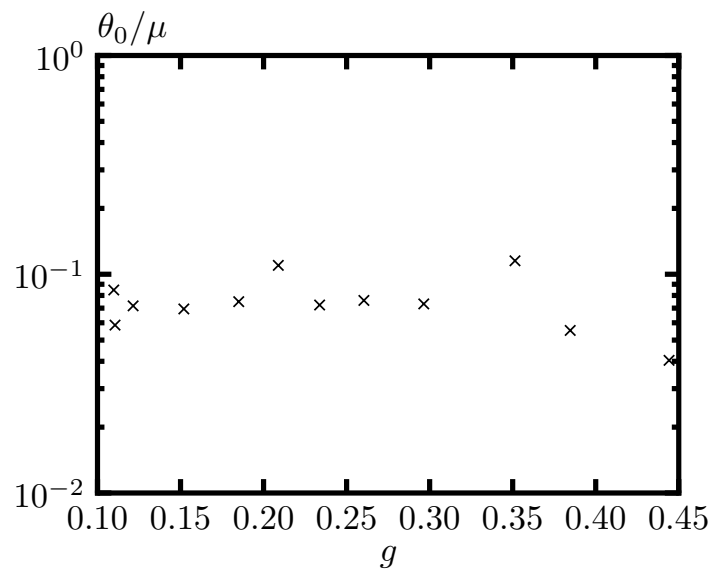

b)

Figure 2.4: Plots of the hardening parameters $\sigma_{\max }$ and $\theta_{0}$ normalized by the temperature dependent shear modulus $\mu$ versus normalized activation energy $g$. Plots are generated using true stress and true strain data.

An extended Voce model

$$
\frac{\sigma_{f}-\sigma_{y}}{\mu}=\sigma_{\max }\left(1-e^{-\varepsilon\left(\theta_{0}+\theta_{\text {soften }}\right) / \sigma_{\max }}\right)-\theta_{\text {soften }} \varepsilon
$$

fits the softening flow curves with $\sigma_{f}$ the flow stress, $\sigma_{y}$ the yield stress, $\mu$ the temperaturedependent shear modulus, $\varepsilon$ the accumulated plastic strain, and $\sigma_{\text {max }}, \theta_{0}$, and $\theta_{\text {soften }}$ temperaturedependent parameters. Figure 2.3 shows a schematic representation of the hardening curve generated by this model. Figures $2.4 \mathrm{a}$ and $\mathrm{b}$ plot $\sigma_{\max }$ and $\theta_{0}$ assuming they are functions of the Kocks-Mecking normalized activation energy. If a test did not significantly harden $\sigma_{\max }=0$ and Fig. 2.4 omits the data point. Figure 2.4a indicates the maximum work hardening stress decreases as the activation energy increases. This indicates an increase in the energy available for thermal recovery mechanisms, thereby lowering the amount of work hardening. Figure 2.4b shows the initial work hardening slope is independent of temperature and strain rate, when normalized by the temperature-dependent shear modulus. This is common in many FCC and BCC metals. The value of this slope is approximately $\theta_{0}=0.075 \mu$.

Figure 2.5 plots the slope $\theta_{\text {soften }}$, normalized by the shear modulus, as a function of g. This figure does not suggest a practical model for $\theta_{\text {soften, }}$, likely because the terminal hardening slope in a tension test is a function of both thermally activated soften mechanisms and the necking in the test specimen. Cyclic tests better separate material softening from geometric localization of the test specimen. However, the plot does indicate an abrupt change in mechanism around $g=0.4$, matching the trend indicated by the yield stresses. 


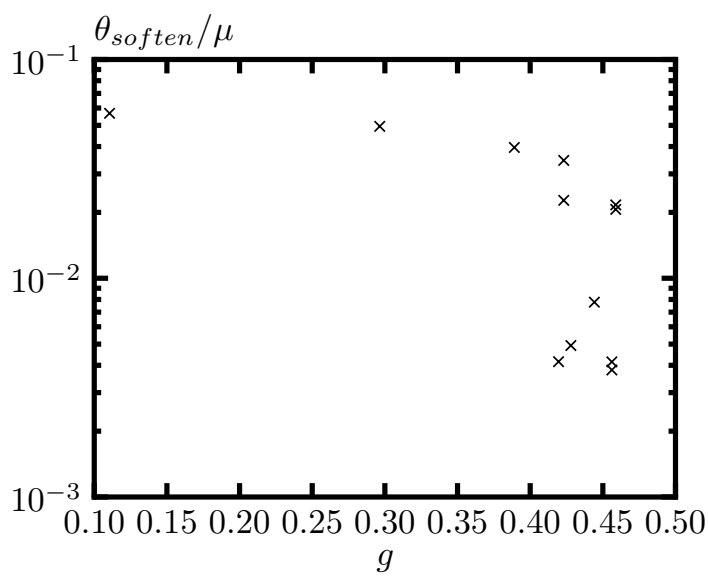

Figure 2.5: Plot of the terminal softening slope $\theta_{\text {soften }} / \mu$ versus normalized activation energy.

\subsubsection{Cyclic experiments}

\subsubsection{Strain controlled cyclic softening}

The prominent feature of Gr. 91 in strain controlled cyclic tests is the development of cyclic softening. As the material accumulates strain the flow stress tends to decrease. This behavior becomes more severe at higher temperatures.

All the collected strain controlled cyclic tests were for fully reversed loading. This subsection focuses on cyclic softening and only considers experiments without a strain hold built into the cycle. Factors affecting the decay of the flow stress, as a function of accumulated cycles or strain, are the temperature and strain rate of the test as well as the strain range of the experiment. Until failure, characterized by a sharp drop in the flow stress, exponential decay from an initial value to a final value well represents most of the data. Figure 2.6 plots the pre-failure saturated flow stress, normalized by the shear modulus, as a function of the normalized activation energy. With the exception of one outlier the data follow a straight line, implying the work softening processes are thermally activated. The amount of work softening increases with increasing activation energy.

\subsubsection{Strain ranges and ratcheting in stress controlled cyclic tests}

Material ratcheting can develop in Gr. 91 uniaxial tests when loaded with non-symmetric cyclic stresses. Some evidence exists for ratcheting even under fully reversed loading, strongly suggesting tension/compression material asymmetry. Creep fatigue experiments incorporating a stress hold at the minimum or maximum stress can increase the amount of material ratcheting. This hold induces preferential creep causing additional, non-symmetric straining that induces ratcheting.

These kind of fatigue experiment provide valuable data on the interaction of creep and plasticity in Gr. 91. However, the results are difficult to summarize. In general, the strain values during each cycle depend on the stress ratio $R$, the hold time on each end of the cycle, 


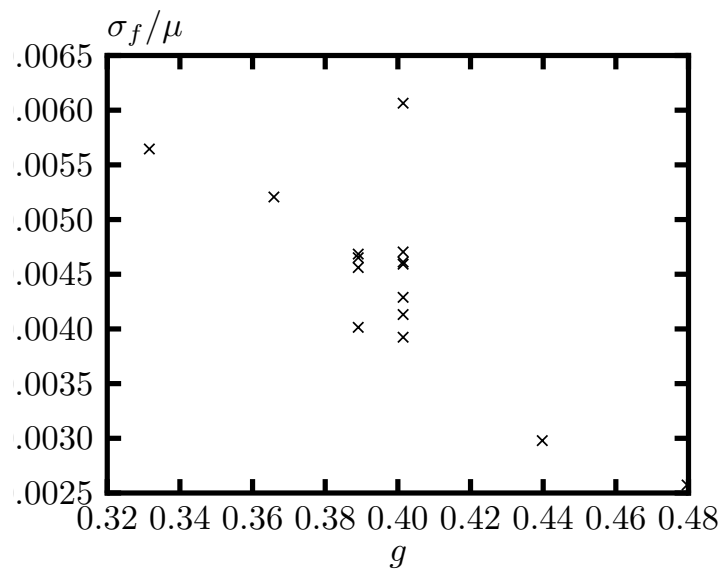

Figure 2.6: Saturated decay stress, normalized by the temperature-dependent shear modulus, versus normalized activation energy.

and the applied stress value in addition to the temperature and loading rate. Baring some simplified method of presenting the data, Figures $2.7 \mathrm{a}$ and b provide full results (maximum strain versus cycle) for groups of similar experiments. Figure 2.7a plots data for tests with $\sigma=400 \mathrm{MPa}, T=550^{\circ} \mathrm{C}, \dot{\sigma}=50 \mathrm{MPa} / \mathrm{s}$, and no holds for different values of the load ratio $R$. Figure $2.7 \mathrm{~b}$ plots results for tests with $\sigma=320 \mathrm{MPa}, T=823 \mathrm{~K}, R=-0.9375$, and no holds for a variety of strain rates. Finally, Fig. 2.7c shows results for a test with $\sigma=320 \mathrm{MPa}, T=550^{\circ} \mathrm{C}, \dot{\sigma}=50 \mathrm{MPa} / \mathrm{s}$ but increasing hold times on the maximum-stress end of the cycle.

\subsubsection{Creep tests}

Kocks and Mecking originally applied their theory to yield and work hardening data from uniaxial tension experiments. As demonstrated in the next section, this form of model reasonably describes the rate sensitivity of deformation for a wider variety of tests, including creep and stress relaxation. Gr. 91 typically exhibits a short period of higher, decreasing creep rates typical of primary creep behavior during the initial part of the creep deformation. It is characterized by the decrease of the creep rate from the initial high value towards a minimum. The time to reach the minimum creep rate is approximately one-third of the creep rupture time over a considerable range of stress levels. Then, after reaching the minimum creep rate, the deformation creep rate in Gr. 91 typically begins increasing again without any significant prolonged period of constant-rate secondary creep. The increase in the creep rate becomes rapid as the final creep rupture is approached. In this regime creep damage, in the form of cavitation, dominates over the deformation due to dislocation motion. However, the Division 5 Code rules would preclude a component reaching this regime under sustained loading. Thus, the creep rates of interest for the consideration of the Kocks-Meeking model would be bounded by the initial primary creep rate and the minimum creep rate. Because the life of in-service components should not extend to failure, primary creep followed by a period of pseudo-secondary creep at the minimum creep rate may sufficiently characterize 
FY17 Status Report on the Initial Development of a Constitutive Model for Grade 91 Steel July 2017
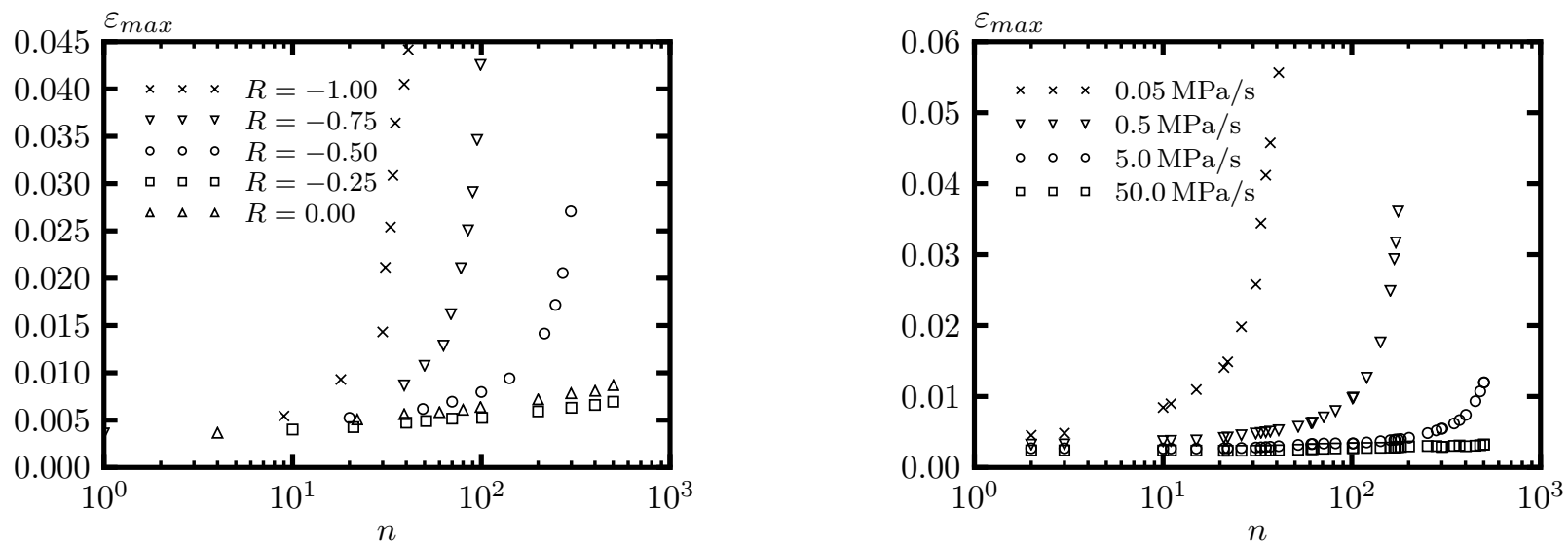

a)

b)

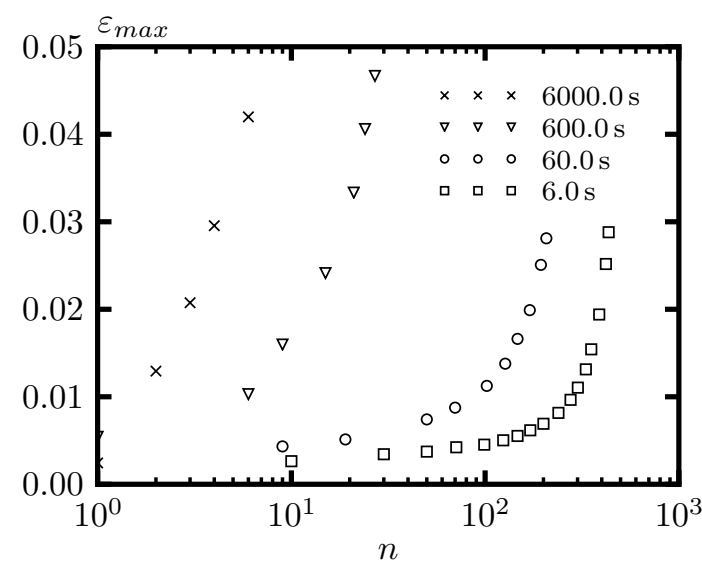

c)

Figure 2.7: Results from a variety of stress controlled cyclic tests. a) Effect of load ratio $R$, b) effect of loading rate $\dot{\sigma}$. 


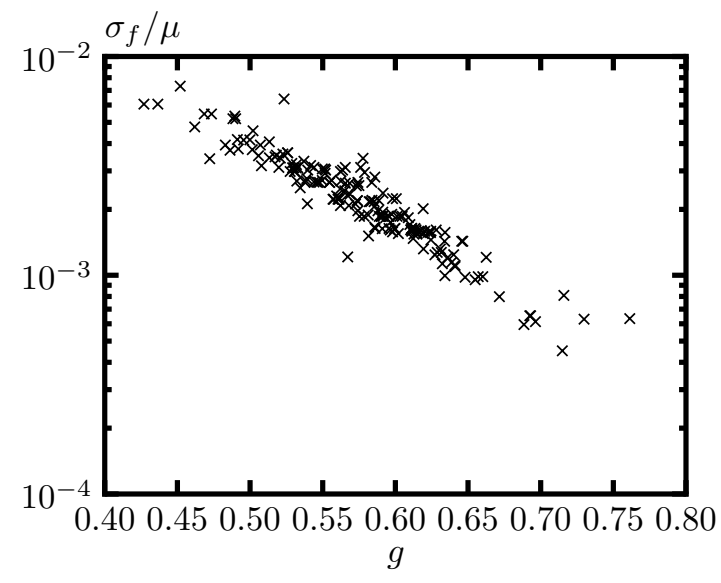

a)

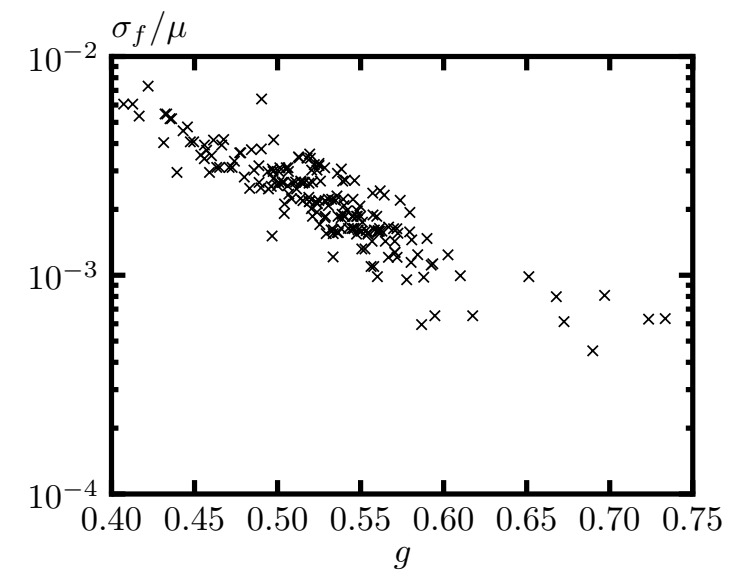

b)

Figure 2.8: Kocks-Mecking plots of the minimum creep rate (a) and initial primary creep rate (b) from the experiments.

the material for design purposes.

Figures 2.8a and 2.8b characterize the initial primary and minimum creep rates from all the creep experiments in the Kocks-Mecking framework. They plot the normalized activation energy, computed with the initial (a) and minimum creep rates (b), versus the applied dead load, normalized by the temperature dependent shear modulus. The plots illustrate the loglinear relation between the normalized activation energy and the creep rate except at high activation energies (high temperatures).

\subsection{Rate sensitivity}

The strain rate sensitivity of the flow stress is critical in the determination of a constitutive model framework. If the material is rate insensitive then the model can separate the contributions of creep and plasticity to inelastic strain and develop separate models for each deformation mechanism. However, if the material is rate sensitive then creep and plasticity will be coupled, requiring a viscoplastic material model representing the interaction between the two mechanisms.

Usually, the equation

$$
\sigma=K \dot{\varepsilon}^{m}
$$

ise used to describe the strain rate sensitivity of some flow stress or back stress $\sigma$ in terms of the rate sensitivity exponent $m$. The temperature and the timescale of the deformation mechanism can affect the instantaneous value of the rate sensitivity exponent. For example, steels are typically considered rate insensitive at room temperature but rate sensitive at elevated temperatures, implying a change in the rate sensitivity exponent at some critical temperature. Furthermore, while a power law is often used to describe both the rate sensitivity of the flow stress in monotonic tension tests and in creep experiments, the rate sensitivity of the deformation mechanisms activated in these different experiments may be 
different. To a large extent, the rate sensitivities of Gr. 91 steel will dictate the form of the constitutive relations.

\subsubsection{In tensile experiments}

Kocks and Mecking developed a framework that unifies the treatment of the rate and temperature dependence of the flow stress in standard metal alloys [40, 38, 39]. Their framework relies on two concepts.

1. The flow stress is proportional to the current, temperature dependent shear modulus. This reflects that the stress required for a dislocation to overcome an obstacle on the microscale generally scales with the current elastic properties of the material. For example, the stress field of a screw dislocation scales with the shear modulus $\mu$.

2. The time required for a dislocation to overcome an obstacle on the microscale governs the rate of plasticity on the macroscale. This time will depend on the energy barrier presented by an obstacle and on the free energy available to the dislocation. This free energy can be expressed in terms of a normalized activation energy $g=(k T) /\left(\mu b^{3}\right) \log \left(\dot{\varepsilon}_{0} / \dot{\varepsilon}\right)$ with $k$ the Boltzmann constant, $T$ the absolute temperature, $b$ the Burgers vector, and $\dot{\varepsilon}_{0}$ some reference strain rate.

Accepting these concepts, the normalized flow stress $\sigma_{f} / \mu$ should scale with the normalized activation energy $g$. Calculating the normalized flow stress requires the shear modulus as a function of temperature. For the analysis, the equation

$$
\mu(T)=\mu_{0}-\frac{D}{\exp \left(T_{0} / T\right)-1}
$$

describes the temperature dependent shear modulus, with $\mu_{0}=88100 \mathrm{MPa}, D=10870 \mathrm{MPa}$, and $T_{0}=331 \mathrm{~K}$. These calibrated material coefficients are a best fit to the temperature dependent Young's modulus values in ASME BPVC Section II, Part D, Table TM and the constant, temperature independent Poisson's ratio $\nu=0.30$. Figure 2.9 compares the Code values to the calibrated model.

Calculating the normalized activation energy requires knowing the reference strain rate $\dot{\varepsilon}_{0}$. The results of the final analysis are typically insensitive to this parameter, provided it is in a physically reasonable range of $\dot{\varepsilon}_{0}>10^{6} \mathrm{~s}^{-1}$. The reference strain rate $\dot{\varepsilon}_{0}=10^{12} \mathrm{~s}^{-1}$ used here was obtained by repeating the Kocks-Mecking analysis used to generate Fig. 2.10 several times and finding the optimal value of $\dot{\varepsilon}_{0}$ that best fits the data to the bilinear form described below. The Kocks-Mecking analysis is extremely insensitive to this parameter for Gr. 91, provided $\dot{\varepsilon}_{0}>10^{7} \mathrm{~s}^{-1}$. For Gr. 91 this analysis uses $b=2.48 \AA$, that of BCC ferrite.

Figure 2.10 shows the results of applying the Kocks-Mecking procedure to two flow stresses: the $0.2 \%$ offset yield stress and the flow stress at $2.5 \%$ strain, using all available tension test data in the final experimental data set. A bilinear functional form, with zero slope at low energies fits the data for both flow stresses. This functional form indicates a clear transition between rate insensitive and rate sensitive deformation at a normalized activation energy of $g=0.4$. The slope of the curves in the rate sensitive regions are $-a=-6.3$ for the yield stress and $-a=-6.8$ for the flow stress at $2.5 \%$ strain. 


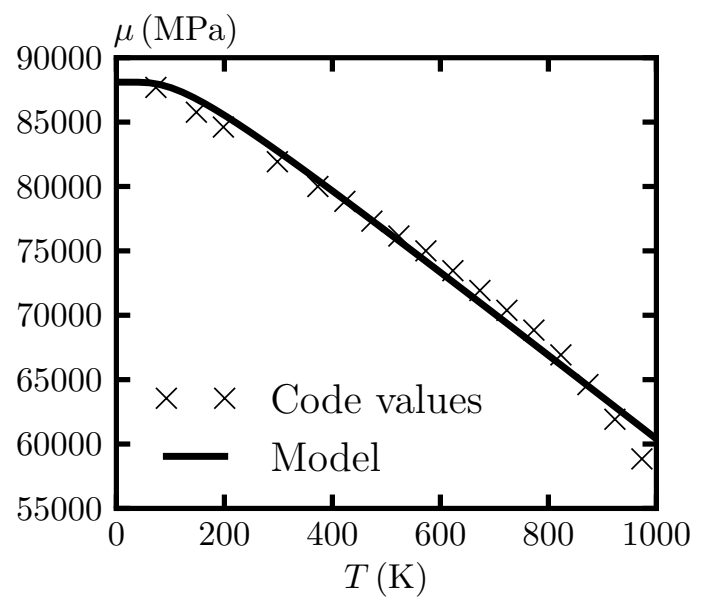

Figure 2.9: Temperature-dependent shear modulus model compared to shear modulus values for Gr. 91 calculated from the ASME BPVC Section II, Part D, Table TM values of Young's modulus and the Section II temperature-independent Poisson's ratio.

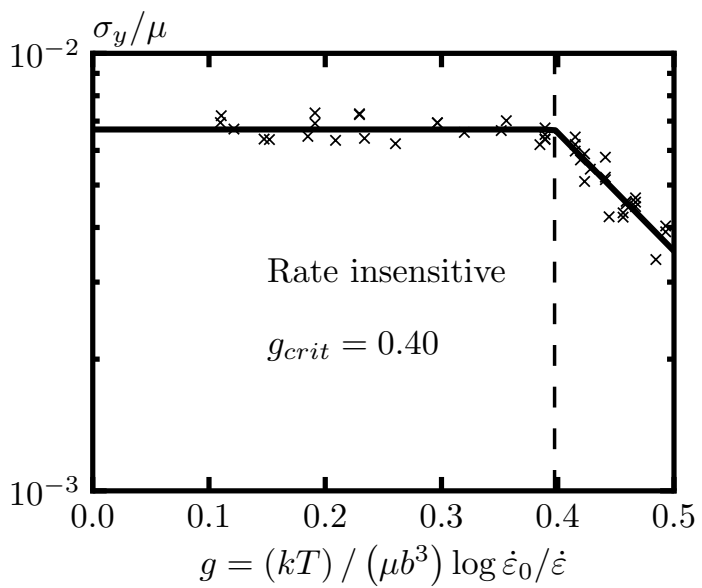

a)

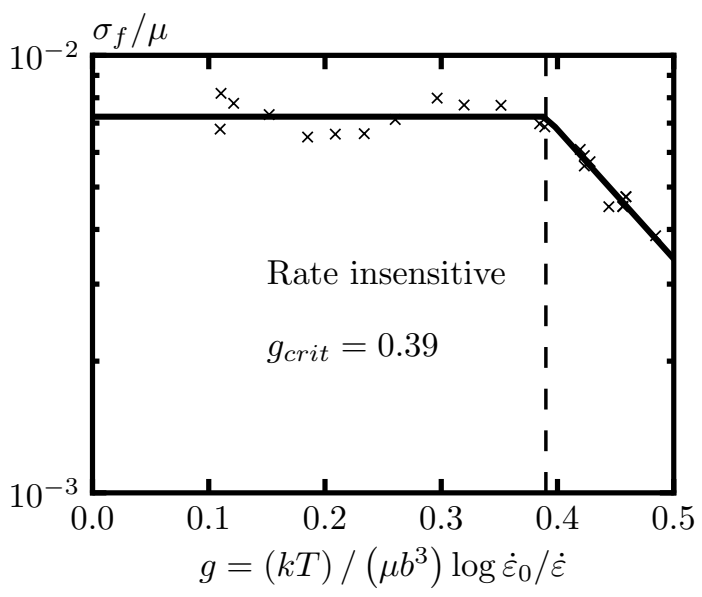

b)

Figure 2.10: Kocks-Mecking, Arrhenius-type analysis of the a) yield and b) flow stress at $2.5 \%$ strain of Gr. 91, using all the identified monotonic data. The graphs plot the stress, normalized by the temperature-dependent shear modulus of the material versus the normalized activation energy. Both plots indicate a transition from rate insensitive deformation to rate sensitive deformation at a normalized activation energy of about $g=0.4$. Both plots use the same reference strain rate $\dot{\varepsilon}_{0}=10^{12} \mathrm{~s}^{-1}$. 
Assuming a typical strain rate of $10^{-6} \mathrm{~s}^{-1}$ for a quasi-static tension test at room temperature, this analysis indicates that Gr. 91 should exhibit rate-independent flow below $T_{\text {crit }} \approx 460^{\circ} \mathrm{C}$. At the extremely low rates typical of creep deformation $\left(10^{-8} \mathrm{~s}^{-1}\right)$ this threshold could decrease to $T_{\text {crit }}=400^{\circ} \mathrm{C}$.

The Kocks-Mecking analysis fits into the usual power law dependency equation. The analysis indicates, in the rate sensitive region,

$$
\log \frac{\sigma}{\mu}=-a \frac{k T}{\mu b^{3}} \log \frac{\dot{\varepsilon}_{0}}{\dot{\varepsilon}}-c
$$

or

$$
\sigma=\mu e^{-c}\left(\frac{\dot{\varepsilon}}{\dot{\varepsilon}_{0}}\right)^{a k T /\left(\mu b^{3}\right)}
$$

implying

$$
\begin{aligned}
m(T) & =\frac{a k T}{\mu b^{3}} \\
K(T) & =\frac{\mu e^{-c}}{\dot{\varepsilon}_{0}^{a k T /\left(\mu b^{3}\right)}}
\end{aligned}
$$

in the usual rate-sensitivity model (Eq. 2.3).

\subsubsection{In creep experiments}

The minimum creep rate is usually described with a power law of the form

$$
\dot{\varepsilon}=C \sigma^{r} e^{-Q /(k T)} \text {. }
$$

Formulating a different power law that describes the temperature and stress dependence reasonably with the form

$$
\dot{\varepsilon}=\dot{\varepsilon}_{0} e^{c \mu b^{3} /(a k T)}\left(\frac{\sigma}{\mu}\right)^{\mu b^{3} /(a k T)}
$$

allows direct compatibility with the Kocks-Mecking formulation.

While this form is functionally equivalent to the Kocks-Mecking formula used above to model the uniaxial tension tests, the actual constants $\dot{\varepsilon}_{0}, a$, and $c$ may not be the same, as the mechanisms controlling rate-dependent flow at the strain rate of a uniaxial tension test may not be the same as the mechanisms causing rate-dependent creep under a constant dead load. To determine what, if any, differences occur between the rate sensitive response of Gr. 91 in the two deformation regimes we can generate plots similar to 2.10 above, now using the minimum creep strain rates and dead load stresses instead of the monotonic stress/strain data.

Figure 2.11 is a Kocks-Mecking plot using the minimum creep strain rates as $\dot{\varepsilon}$ and the applied dead load stresses as $\sigma$. Some experiments were excluded from this plot of their creep strain versus time data was too scattered to calculated a reasonable minimum creep rate via finite differencing. This typically occurred if there were too few data points in the digitized creep curve. Here $\dot{\varepsilon}_{0}$ was optimized to find the best single line fit to the data, as opposed 


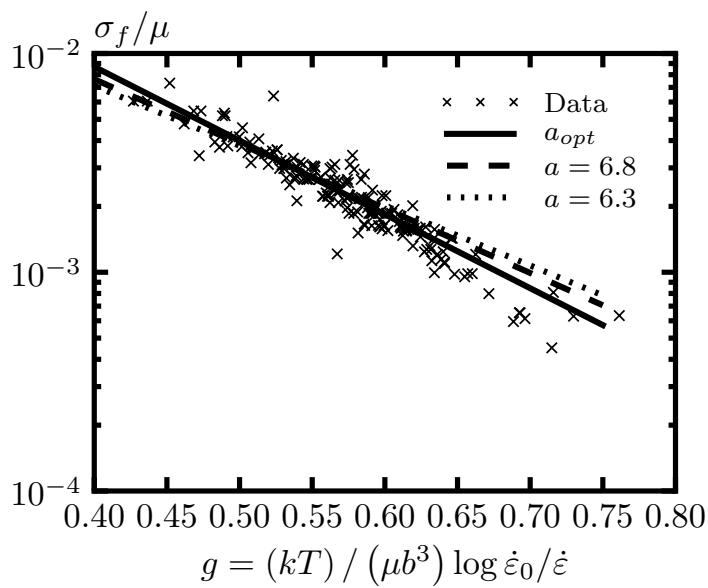

Figure 2.11: Kocks-Mecking plot of the minimum creep rates from 96 experiments.

to the bilinear form used earlier. As before, the fit is insensitive to the value of $\dot{\varepsilon}_{0}$ so, as before, $\dot{\varepsilon}_{0}=10^{12} \mathrm{~s}^{-1}$. Three lines show various model fits, using Eq. 2.10 and this reference strain rate. The solid line shows the model with optimal fit parameters $(-a=-7.7)$ while the two dashed lines show fits with the slopes fixed to the optimal slopes for the yield stress $(-a=-6.3)$ and flow stress at $2.5 \%$ strain $(-a=-6.8)$ but the intercept varied to produce an optimal fit. All three curves fit the data well, implying only two regimes of rate-sensitivity: rate insensitive deformation $(a=0)$ at low normalized activation energies $(g<0.4)$ and a regime of rate sensitive deformation at higher activation energies with slope $a$ in the range of -6.3 to -7.7 .

\subsubsection{Combined model}

Work hardening complicates combining the three previous Kocks-Mecking analyses (yield stress, flow stress at $2.5 \%$ strain, and creep stress) into a single figure. Kocks-Mecking analysis relates the macroscale stress required to overcome some set of microstructural obstacles (i.e. a dislocation structure) to the normalized activation energy the population of glissile dislocations can use to slip past those obstacles. Presumably each sample in each experiment has a different obstacle distribution because of its different history. However, assuming reasonable similarity in processing between different heats of material, the yield stress in the uniaxial tests represents the flow stress required to overcome some standardized set of obstacles - the as-delivered, initial microstructure. Without adopting a model for work hardening we cannot reduce the other two cases to a standardized obstacle density and so, strictly, should not assume the stresses in these cases represent the stress required to overcome a particular obstacle distribution.

However, creep tests are typically performed at stress levels less than or very close to the yield stress, meaning there will be little change in the microstructure from the as-delivered structure before creep begins. Reasonably then creep tests can be included along with the uniaxial tension yield data, as both begin from (approximately) the initial microstructure. Furthermore, stress relaxation test data can also be included assuming relaxation begins 


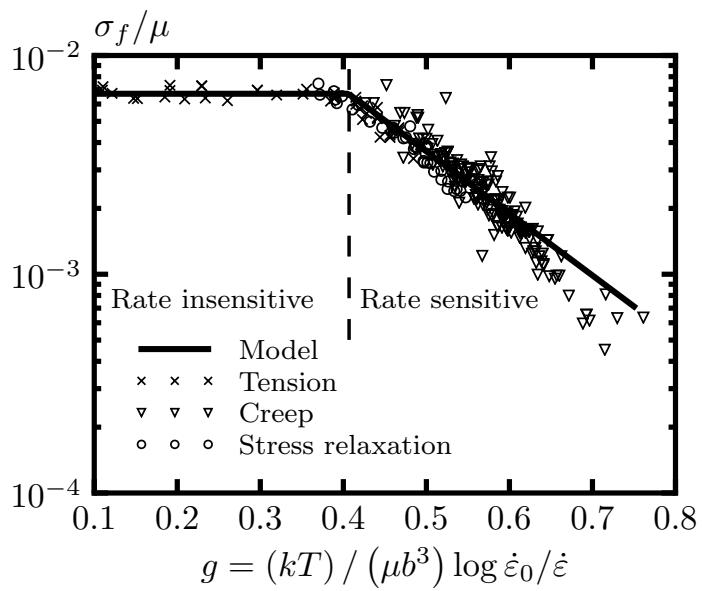

Figure 2.12: Kocks-Mecking plot combining data from the uniaxial tension and creep experiments.

after only limited work hardening. For the stress relaxation tests we must adopt a different strain rate to calculate the normalized activation energy, as the total strain rate in stress relaxation tests is zero. Assuming a partition of strain into

$$
\varepsilon=\varepsilon_{e}+\varepsilon_{i n}
$$

where $\varepsilon_{\text {in }}$ lump all inelastic contributions, then

$$
\begin{gathered}
\dot{\varepsilon}=\dot{\varepsilon}_{e}+\dot{\varepsilon}_{i n}=0 \\
\dot{\varepsilon}_{e}=-\dot{\varepsilon}_{i n}
\end{gathered}
$$

and we reasonably adopt the rate of elastic strain as the Kocks-Mecking strain rate. This elastic strain rate is

$$
\dot{\varepsilon}_{e}=\frac{\dot{\sigma}}{E}
$$

which can be computed from the stress relaxation rate. Each stress relaxation test then provides multiple data points for Kocks-Mecking analysis, as each test provides multiple $\left(\sigma, \dot{\varepsilon}_{e}\right)$ pairs. These data are culled to provide a limited number of pairs, evenly log-spaced across the strain rate range probed by the experiment.

Figure 2.12 then combines the yield stress, creep test, and stress relaxation data, including data from 44 uniaxial tension tests, 193 creep tests, and 10 stress relaxation tests. This plot is a master curve for the rate sensitivity of Gr. 91. It shows that all the data considered so far, excepting outliers, fall onto a bilinear curve with rate insensitive and rate sensitive regimes. Again, this curve uses $\dot{\varepsilon}_{0}=10^{12} \mathrm{~s}^{-1}$.

The final, fitted transition point for the combined dataset is $g_{\text {crit }}=0.41$ and the slope of the rate-sensitive region is $a=6.5$.

Using a value of $g_{\text {crit }}=0.41$ Fig. 2.13 plots the boundary between rate-independent and rate-dependent behavior in terms of temperature and strain rate. Figure 2.13a shows a full temperature range while Fig. $2.13 \mathrm{~b}$ zooms in to the range where the strain rates 


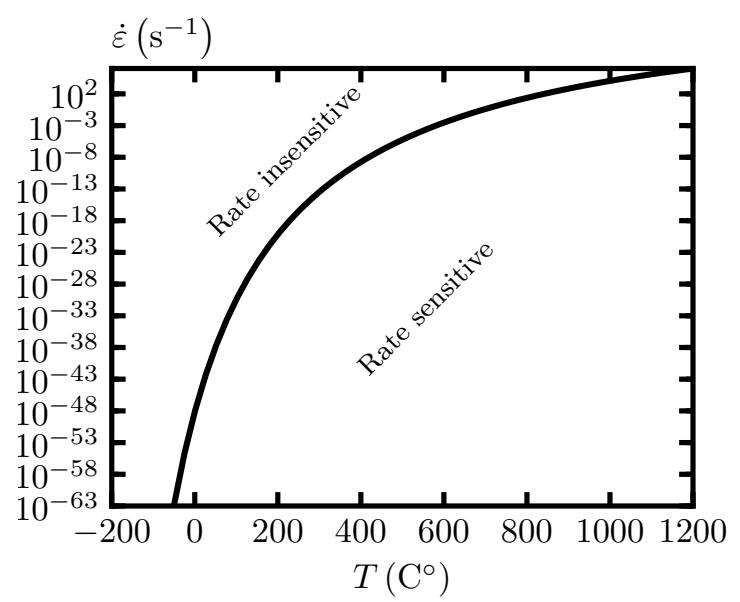

a)

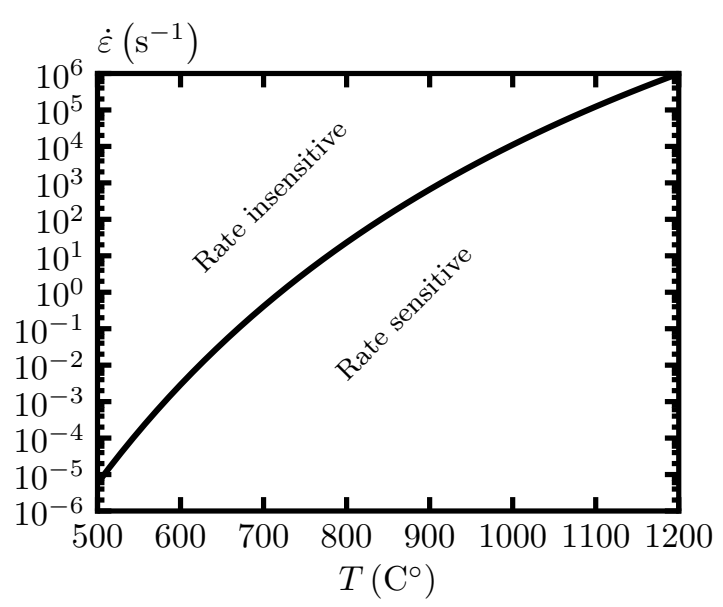

b)

Figure 2.13: Plots show the regions of rate sensitivity for Gr. 91 in terms of temperature and strain rate. a) shows a full temperature range and b) zooms into the temperature range where the corresponding strain rates are experimentally accessible.

are experimentally accessible. This plot predicts the material will be rate-insensitive for all accessible strain rates until approximately $450^{\circ} \mathrm{C}$, which is in agreement with the previous results using only the tensions tests.

Figure 2.14 plots the classical rate sensitivity exponent $m$ from Eqs. 2.3 and 2.7 versus temperature. This plot only shows the rate sensitivity implied by a Kocks-Mecking model with slope $a=6.5$. The plot does not reflect the mechanism change at $g_{\text {crit }}=0.41$.

A later chapter develops a method for converting the experimental master curve into a numerical constitutive model for material rate sensitivity. 
FY17 Status Report on the Initial Development of a Constitutive Model for Grade 91 Steel July 2017

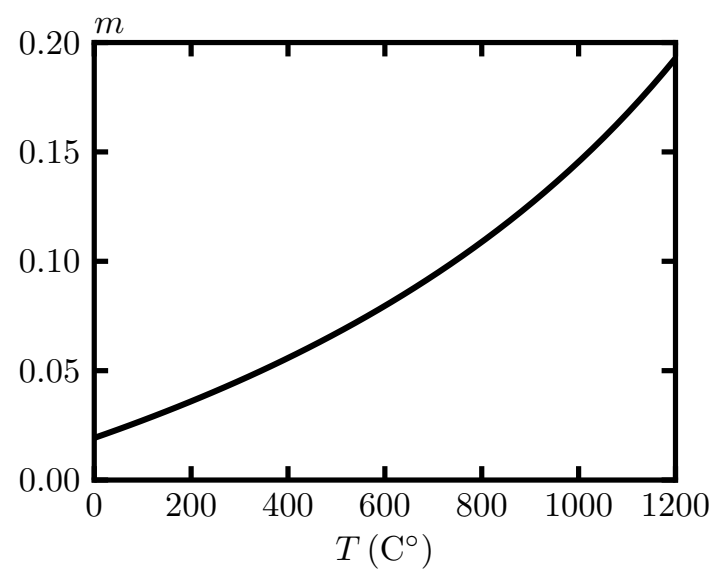

Figure 2.14: Plot of temperature dependent rate sensitivity exponent $m$ implied by the Kocks-Mecking model with $a=6.5$. 


\section{Viscoplastic material models}

\subsection{General framework}

Small strain theory is adequate to comply with the Division 5 rules for Level A and B operations. Therefore, models treat the total strain as small and additively decomposed into a contribution from elasticity and an inelastic contribution

$$
\dot{\varepsilon}=\dot{\varepsilon}_{e}+\dot{\varepsilon}_{i n}
$$

where $\dot{\varepsilon}$ describes the total small strain rate, $\dot{\varepsilon}_{e}$ is the elastic strain rate, and $\dot{\varepsilon}_{i n}$ represents the contribution of coupled creep-plasticity. The elastic strains remain small for engineering metals and are reasonably related to the stresses via Hooke's law so the model becomes

$$
\dot{\boldsymbol{\sigma}}=\mathbf{C}:\left(\dot{\varepsilon}-\dot{\varepsilon}_{i n}\right)
$$

with $\mathbf{C}$ the temperature-dependent elastic stiffness tensor and $\dot{\boldsymbol{\sigma}}$ the stress rate. On the macroscale Gr. 91 is elastically isotropic and previous work establishes the temperature dependence of the elastic constants. Therefore, a macroscale constitutive model for Gr. 91 reduces to choosing a model for the inelastic strain rates $\dot{\varepsilon}_{i n}$.

Many of the models for Gr. 91 identified in the literature are Perzyna formulations where the inelastic strain rates under isothermal conditions take the form

$$
\dot{\boldsymbol{\varepsilon}}_{i n}=\mathbf{g}(\boldsymbol{\sigma}, \mathbf{q}, T) \dot{p}(\boldsymbol{\sigma}, \mathbf{q}, T)=\mathbf{g}(\boldsymbol{\sigma}, \mathbf{q}, T)\left\langle\frac{f(\boldsymbol{\sigma}, \mathbf{q}, T)}{D(T)}\right\rangle^{n(T)}
$$

where $\mathbf{g}$ is the direction of inelastic flow, as a function of stress, some set of evolving internal variables $\mathbf{q}$, and temperature, $\dot{p}$ is the rate of plastic deformation as a function of the same quantities, $f$ is a function, generally describing some reference yield surface, $D$ is the material fluidity, and $n$ the material rate sensitivity. Furthermore, all the identified models take on the specific form of a Perzyna formulation based on $J_{2}$ plasticity with combined isotropic and kinematic hardening. The equation

$$
\dot{\varepsilon}_{i n}=\frac{\boldsymbol{\sigma}-\mathbf{X}(\mathbf{q})}{J_{2}(\boldsymbol{\sigma}-\mathbf{X}(\mathbf{q}))}\left\langle\frac{J_{2}(\boldsymbol{\sigma}-\mathbf{X}(\mathbf{q}))-\sigma_{i s o}(\mathbf{q})}{D(T)}\right\rangle^{n(T)}
$$

describes all identified models where $J_{2}$ is the second deviatoric stress invariant, $\mathbf{X}$ is some backstress describing kinematic hardening, and $\sigma_{i s o}$ is an isotropic hardening parameter, both functions of the internal variables. In the context of strain controlled, one dimensional cyclic plasticity isotropic hardening represents a uniform expansion or contraction of the hysteresis loop while kinematic hardening represents a shifting or skewing of the loop due to ratcheting or the Bauschinger effect. A model for Gr. 91 requires some description of kinematic hardening to account for ratcheting deformation and the evolution of the hysteresis loop with cyclic loading, while the various models described below do not have a uniform interpretation of isotropic hardening. Some models use the isotropic term to represent work softening, some use it to represent material effects like dynamic strain aging, and some omit isotropic hardening entirely. 
Nearly all empirical models for viscoplasticity fall into the framework described here. These models are empirical because the kinematic hardening backstress, the isotropic hardening parameter, and the use of the $J_{2}$ invariant do not have immediate physical interpretations. Furthermore, this general form does not describe how the model parameters and the evolution of the state should change with temperature. Without such a theory the models, with one exception, either fit different sets of constants for different temperatures or provide highly empirical interpolation formulas relating the temperature to the model parameters. However, despite lacking a direct connection to the lower scale physics, this form of model has been used successfully many times to predict the deformation of engineering structures under thermo-mechanical cyclic load.

\subsection{Gr. 91 material models in the literature}

The only difference between the various models in the literature are the choice of the internal variable set $\mathbf{q}$, the ordinary differential equations describing the evolution of the state $\dot{\mathbf{q}}(\boldsymbol{\sigma}, \mathbf{q}, T)$, the maps $\mathbf{X}(\mathbf{q})$ and $\sigma_{\text {iso }}(\mathbf{q})$ taking the state to the kinematic and isotropic hardening variables, and the temperature dependence of the model parameters. The majority of the models adopt the form described by Chaboche [14, 6, 11, 7, 8, 16, 9, 12, 15, 13, 10], with some slight modifications, for the kinematic hardening backstress $\mathbf{X}$

$$
\begin{aligned}
\mathbf{X} & =\sum_{i=1}^{n_{x}} \mathbf{X}_{i} \\
\dot{\mathbf{X}}_{i} & =C_{i}\left(\frac{2}{3} a_{i} \mathbf{g}-\mathbf{X}_{i}\right) \dot{p}-\gamma_{i} J_{2}\left(\mathbf{X}_{i}\right)^{m-1} \mathbf{X}_{i}
\end{aligned}
$$

where $C_{i}, a_{i}, \gamma_{i}$, and $m$ are all temperature-dependent empirical parameters. Furthermore, the majority of the models adopt a Voce form for the isotropic hardening

$$
\dot{\sigma}_{i s o}=R\left(q-\sigma_{i s o}\right) \dot{p} .
$$

This form assumes isothermal conditions. The differences between models are the temperature dependence of the parameters, the number of backstresses and the presence or absence of the static recovery term on each backstress, the initial conditions for the state, and tweaks to the basic formulation - for example, making some state evolution constants evolve with time.

Table 3.1 summarizes five categories or families of models identified in the literature. These are grouped by family because often the same authors or a subset of the authors revisited and revised the model in later publications. Where possible this report analyzes the latest version of each model family. The table briefly describes the model in the framework given above. All the models follow the form outlined in 3.1 and most follow a generalized Chaboche form. For the Chaboche models the table contains a brief description of the scheme used to vary the model parameters with temperature and a brief description of any changes from the basic Chaboche form described by Eqs. 3.5, 3.6, and 3.7.

Two of the models do not fit directly into the Chaboche framework. Moosbrugger [50] was the only author to provide some physical motivation for how the model parameters scale 
FY17 Status Report on the Initial Development of a Constitutive Model for Grade 91 Steel July 2017

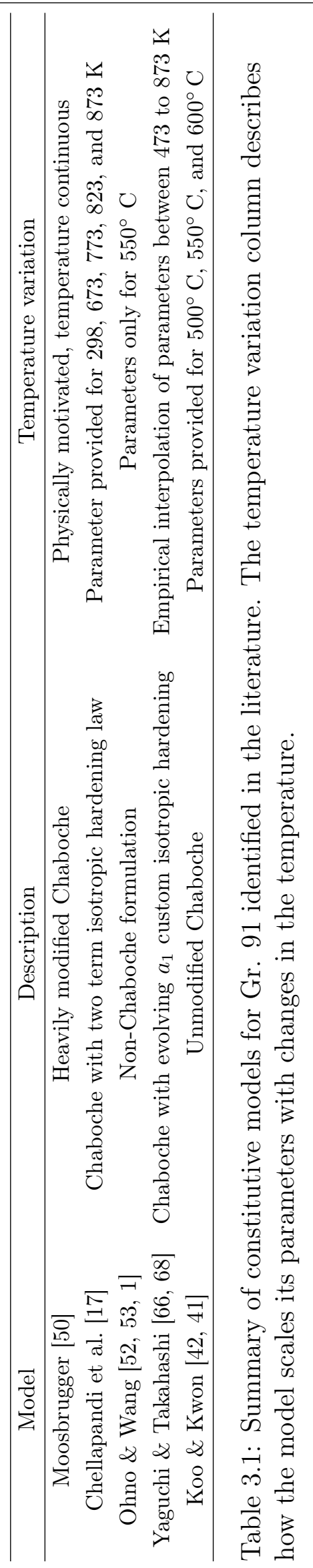


with temperature. His model allows for simulations over a wide temperature range, from room temperature to above $600^{\circ} \mathrm{C}$. The evolution of the backstresses in the Ohno \& Wang formulation $[52,53,1]$ uses a rival framework to that of Chaboche. Ohno \& Wang models evolve the backstress based on a critical state of dynamic recovery.

Chellapandi's model is based on Moosburgger's, recasting the model into a conventional Chaboche form with parameters provided at discrete temperatures. The version of the Yaguchi \& Takahashi model considered in this report is that of [66], extended to three dimensions in [68] and not the improved model developed in [68] . Unfortunately Yaguchi \& Takahashi only provided material coefficients for one temperature for the improved version.

\subsection{Model evaluation}

The suitability criteria developed at the start of this chapter eliminate the Ohno \& Wang and improved Yaguchi \& Takahashi model from contention. These two models only provide calibrated coefficients for $550^{\circ} \mathrm{C}$ meaning the formulations cannot be used for design situations with fluctuating thermal loads. Unfortunately, practical implementational issues also eliminate the Moosburgger and Chellapandi models. There are severe typographical errors in both manuscripts, meaning the printed model parameters do not correspond to the results published in the studies. These issues could not be overcome via personal communication with Moosburgger.

This leaves only the Koo \& Kwon and Yaguchi \& Takahashi models. These models are fairly similar, both having the Chaboche form for the evolution of the backstress. The Koo \& Kwon model is an unmodified Chaboche formulation and uses the Voce isotropic hardening term to represent cyclic softening. Yaguchi \& Takahash modified the Chaboche form by having an evolving $a_{1}$ constant in the backstress evolution to account for cyclic softening. They use a custom isotropic hardening model to account for dynamic strain aging effects. The next chapter evaluates these two models by implementing full, 3D versions and comparing results from the implemented models to the experimental database described in Chapter 2. 


\section{Implementation and performance of the Koo and Yaguchi models}

This chapter describes the implementation and testing of two viscoplatic models for Gr. 91. The models are tested against the data set described in Chapter 2, using the reduced set of plots describing the data developed in Section 2.2. This requires running a large quantity of material point simulations corresponding to the conditions of each experiment. After running these simulations the data are collected into the same series of plots found in Section 2.2, now including data series showing the model results overlayed on the points describing the experiments.

The two models selected for implementation are Koo and Lee [42] and Yaguchi and Takahashi [66]. Essentially these models were selected from the five identified in Chapter 3 because it is feasible to implement them in their full 3D form from the information available in the publications describing their development. Both models were implemented into a common material model library so that they have the same interface. To generate the data required for the comparison plots drivers exercise the models under the correct experimental conditions. Depending on the type of experimental loading, a driver will control the temperature and either stress or strain history of a material point. Stress control requires inverting the constitutive equations outlined below with Newton's method. This required the implementation of five types of drivers: uniaxial tension, strain-controlled cyclic, stress-controlled cyclic, creep, and stress-relaxation. The common material interface also simplifies the task of later integrating one or both of these models into a finite element solver.

The model implementations were verified by comparing the results of simulations against model results published along with the model description and by verifying the various derivatives required for the implementation of the models with implicit integration using finite differencing. Furthermore, the material model library implements the algorithmic tangent required for quadratic convergence of the material point drivers and, later, an implicit finite element solver. This required some development of both models beyond the information presented in the corresponding publications.

The integration of both models and the calculation of the tangent follows a common format. The rate equations

$$
\begin{array}{r}
\dot{\boldsymbol{\sigma}}=\dot{\boldsymbol{\sigma}}(\boldsymbol{\sigma}, \mathbf{q}, \dot{\boldsymbol{\varepsilon}}, T, \dot{T}, t) \\
\dot{\mathbf{q}}=\dot{\mathbf{q}}(\boldsymbol{\sigma}, \mathbf{q}, \dot{\boldsymbol{\varepsilon}}, T, \dot{T}, t)
\end{array}
$$

describe any viscoplastic model in terms of two functions of the stress $\boldsymbol{\sigma}$, internal variables $\mathbf{q}$, strain rate $\dot{\varepsilon}$, temperature $T$, temperature rate $\dot{T}$, and time $t$. One function describes the stress rate $\dot{\boldsymbol{\sigma}}$ while the other describes the evolution of the internal variables with time $(\dot{\mathbf{q}})$. Both implemented models can be described with this form.

The stress update is an implicit integration of the two, coupled differential equations:

$$
\begin{aligned}
& \mathbf{R}_{1}=-\boldsymbol{\sigma}_{n+1}+\boldsymbol{\sigma}_{n}+\dot{\boldsymbol{\sigma}}\left(\boldsymbol{\sigma}_{n+1}, \mathbf{q}_{n+1}, \dot{\boldsymbol{\varepsilon}}_{n+1}, T_{n+1} \dot{T}_{n+1},, t_{n+1}\right) \Delta t_{n+1}=\mathbf{0} \\
& \mathbf{R}_{2}=-\mathbf{q}_{n+1}+\mathbf{q}_{n}+\dot{\mathbf{q}}\left(\boldsymbol{\sigma}_{n+1}, \mathbf{q}_{n+1}, \dot{\boldsymbol{\varepsilon}}_{n+1}, T_{n+1}, \dot{T}_{n+1}, t_{n+1}\right) \Delta t_{n+1}=\mathbf{0}
\end{aligned}
$$


Solving these residual equations gives the stresses and internal variables at the next time step. The implementation solves the nonlinear equations via Newton's method, requiring the Jacobian given by

$$
\mathbf{J}=\left[\begin{array}{cc}
\mathbf{J}_{\sigma \sigma} & \mathbf{J}_{\sigma q} \\
\mathbf{J}_{q \sigma} & \mathbf{J}_{q q}
\end{array}\right]=\left[\begin{array}{cc}
-\mathbf{I}+\frac{\partial \dot{\boldsymbol{\sigma}}_{n+1}}{\partial \boldsymbol{\sigma}_{n+1}} \Delta t_{n+1} & \frac{\partial \dot{\boldsymbol{\sigma}}_{n+1}}{\partial \boldsymbol{q}_{n+1}} \Delta t_{n+1} \\
\frac{\partial \dot{\mathbf{q}}_{n+1}}{\partial \boldsymbol{\sigma}_{n+1}} \Delta t_{n+1} & -\mathbf{I}+\frac{\partial \dot{\mathbf{q}}_{n+1}}{\partial \boldsymbol{q}_{n+1}} \Delta t_{n+1}
\end{array}\right]
$$

The algorithmic tangent in this context of small strains is the derivative $\mathbf{T}_{n+1}=d \boldsymbol{\sigma}_{n+1} / d \boldsymbol{\varepsilon}_{n+1}$. The implementation solves for this derivative as an implicit function of derivative quantities already computed for the Jacobian calculation described by Eq. 4.5. Assuming Newton's method proceeds to true convergence, where $\mathbf{R}_{1}=\mathbf{0}$ and $\mathbf{R}_{2}=\mathbf{0}$ then

$$
\begin{aligned}
& d \mathbf{R}_{1}=\frac{\partial \mathbf{R}_{1}}{\partial \boldsymbol{\sigma}_{n+1}}: d \boldsymbol{\sigma}_{n+1}+\frac{\partial \mathbf{R}_{1}}{\partial \mathbf{q}_{n+1}}: d \mathbf{q}_{n+1}+\frac{\partial \mathbf{R}_{1}}{\partial \boldsymbol{\varepsilon}_{n+1}}: d \dot{\boldsymbol{\varepsilon}}_{n+1}+\frac{\partial \mathbf{R}_{1}}{\partial T_{n+1}}: d T_{n+1}+\frac{\partial \mathbf{R}_{1}}{\partial t_{n+1}}: d t_{n+1}=\mathbf{0} \\
& d \mathbf{R}_{2}=\frac{\partial \mathbf{R}_{2}}{\partial \boldsymbol{\sigma}_{n+1}}: d \boldsymbol{\sigma}_{n+1}+\frac{\partial \mathbf{R}_{2}}{\partial \mathbf{q}_{n+1}}: d \mathbf{q}_{n+1}+\frac{\partial \mathbf{R}_{2}}{\partial \boldsymbol{\varepsilon}_{n+1}}: d \dot{\boldsymbol{\varepsilon}}_{n+1}+\frac{\partial \mathbf{R}_{2}}{\partial T_{n+1}}: d T_{n+1}+\frac{\partial \mathbf{R}_{2}}{\partial t_{n+1}}: d t_{n+1}=\mathbf{0}
\end{aligned}
$$

Applying the implicit function theorem yields

$$
\begin{gathered}
\mathbf{J}_{\sigma \sigma}: \frac{d \boldsymbol{\sigma}_{n+1}}{d \dot{\boldsymbol{\varepsilon}}_{n+1}}+\mathbf{J}_{\sigma q}: \frac{d \mathbf{q}_{n+1}}{d \dot{\boldsymbol{\varepsilon}}_{n+1}}+\frac{\partial \mathbf{R}_{1}}{\partial \dot{\boldsymbol{\varepsilon}}_{n+1}}+\frac{\partial \mathbf{R}_{1}}{\partial T_{n+1}}: \frac{d T_{n+1}}{d \dot{\boldsymbol{\varepsilon}}_{n+1}}+\frac{\partial \mathbf{R}_{1}}{\partial \dot{T}_{n+1}}: \frac{d \dot{T}_{n+1}}{d \dot{\boldsymbol{\varepsilon}}_{n+1}}+\frac{\partial \mathbf{R}_{1}}{\partial t_{n+1}}: \frac{d t_{n+1}}{d \dot{\boldsymbol{\varepsilon}}_{n+1}}=\mathbf{0} \\
\mathbf{J}_{q \sigma}: \frac{d \boldsymbol{\sigma}_{n+1}}{d \dot{\boldsymbol{\varepsilon}}_{n+1}}+\mathbf{J}_{q q}: \frac{d \mathbf{q}_{n+1}}{d \dot{\boldsymbol{\varepsilon}}_{n+1}}+\frac{\partial \mathbf{R}_{2}}{\partial \dot{\boldsymbol{\varepsilon}}_{n+1}}+\frac{\partial \mathbf{R}_{2}}{\partial T_{n+1}}: \frac{d T_{n+1}}{d \dot{\boldsymbol{\varepsilon}}_{n+1}}+\frac{\partial \mathbf{R}_{2}}{\partial \dot{T}_{n+1}}: \frac{d \dot{T}_{n+1}}{d \dot{\boldsymbol{\varepsilon}}_{n+1}}+\frac{\partial \mathbf{R}_{2}}{\partial t_{n+1}}: \frac{d t_{n+1}}{d \dot{\boldsymbol{\varepsilon}}_{n+1}}=\mathbf{0} .
\end{gathered}
$$

This system of tensor equations can be solved for the algorithmic tangent. Assuming

1. there is no explicit time dependence or the time dependence is small compared to the dependence on the physical variables

2. the temperature change is gradual

greatly simplifies the equations to

$$
\begin{aligned}
& \mathbf{J}_{\sigma \sigma}: \mathbf{A}_{n+1}+\mathbf{J}_{\sigma q}: \frac{d \mathbf{q}_{n+1}}{d \dot{\varepsilon}_{n+1}}+\frac{\partial \mathbf{R}_{1}}{\partial \dot{\varepsilon}_{n+1}}=\mathbf{0} \\
& \mathbf{J}_{q \sigma}: \mathbf{A}_{n+1}+\mathbf{J}_{q q}: \frac{d \mathbf{q}_{n+1}}{d \dot{\varepsilon}_{n+1}}+\frac{\partial \mathbf{R}_{2}}{\partial \dot{\varepsilon}_{n+1}}=\mathbf{0}
\end{aligned}
$$

with $\mathbf{A}_{n+1}=\frac{d \boldsymbol{\sigma}_{n+1}}{d \dot{\varepsilon}_{n+1}}=\Delta t_{n+1} \mathbf{T}_{n+1}$. Solving for the tangent analytically gives

$$
\begin{aligned}
& \mathbf{X}=\frac{\partial \dot{\boldsymbol{\sigma}}_{n+1}}{\partial \dot{\boldsymbol{\varepsilon}}_{n+1}} \Delta t_{n+1} \\
& \mathbf{Y}=\frac{\partial \dot{\mathbf{q}}_{n+1}}{\partial \dot{\boldsymbol{\varepsilon}}_{n+1}} \Delta t_{n+1}
\end{aligned}
$$




$$
\mathbf{T}_{n+1}=\frac{1}{\Delta t_{n+1}}\left(\mathbf{J}_{\sigma \sigma}-\mathbf{J}_{\sigma q}: \mathbf{J}_{q q}^{-1}: \mathbf{J}_{q \sigma}\right)^{-1}:\left(\mathbf{J}_{\sigma q}: \mathbf{J}_{q q}^{-1}: \mathbf{Y}-\mathbf{X}\right)
$$

This method for calculating the tangent is convenient because it applies to any viscoplastic material model. Using the integration algorithm outlined above, a model is completely defined by the functions $\dot{\boldsymbol{\sigma}}$ and $\dot{\mathbf{q}}$, along with the partial derivatives of these functions with respect to $\boldsymbol{\sigma}, \mathbf{q}, \dot{\boldsymbol{\varepsilon}}, t$, and $T$. The accuracy of the final integration algorithm was verified by computing the algorithmic tangent at each step of an arbitrary strain history twice, once via this integration algorithm and once by finite differencing. Both method produce identical results to the limit of the accuracy of the finite difference method.

This method of integration differs somewhat from the classical methods found in Simo and Hughes [60]. Typically, plasticity integration algorithms are described by a plastic flow rule, a hardening rule, and, in the case of viscoplasticity, an equation for the plastic flow rate:

$$
\begin{aligned}
\dot{\boldsymbol{\varepsilon}}^{p} & =\dot{\gamma} \mathbf{g}(\boldsymbol{\sigma}, \boldsymbol{\alpha}) \\
\dot{\boldsymbol{\alpha}} & =\dot{\gamma} \mathbf{h}(\boldsymbol{\sigma}, \boldsymbol{\alpha}) \\
\dot{\gamma} & =y(\boldsymbol{\sigma}, \boldsymbol{\alpha}) .
\end{aligned}
$$

This form is a subset of the general method described here. The equations

$$
\begin{array}{lr}
\dot{\boldsymbol{\sigma}}=\mathbf{C}:(\dot{\boldsymbol{\varepsilon}}-y \mathbf{g}) \\
\dot{\mathbf{q}}= & y \mathbf{h}
\end{array}
$$

with $\mathbf{C}$ the elastic tensor establish the connection between the two formulations.

\subsection{Koo \& Kwon model}

The model presented in Koo and Kwon [41] for Gr. 91 is an unmodified Chaboche model with three backstresses and without hardening memory terms [46]. The internal variable set is

$$
\mathbf{q}=\left[\begin{array}{c}
\sigma_{F} \\
\mathbf{X}_{1} \\
\mathbf{X}_{2} \\
\mathbf{X}_{3}
\end{array}\right]
$$

with $\sigma_{F}$ the isotropic hardening variable and $\mathbf{X}=\sum_{i=1}^{3} \mathbf{X}_{i}$ the Chaboche backstress. The flow rule is associative to the von Mises yield surface

$$
\begin{gathered}
f=\|\mathbf{s}+\mathbf{X}\|+\sqrt{\frac{2}{3}} \sigma_{F} \\
\mathbf{g}=\frac{\partial f}{\partial \boldsymbol{\sigma}} .
\end{gathered}
$$

Isotropic hardening has a Voce form

$$
\dot{\sigma}_{F}=b\left[Q-\sigma_{F}\right] y
$$


FY17 Status Report on the Initial Development of a Constitutive Model for Grade 91 Steel

July 2017

\begin{tabular}{cccccccccccc}
\hline & $E$ & $C_{1}$ & $C_{2}$ & $C_{3}$ & $\gamma_{1}$ & $\gamma_{2}$ & $\gamma_{3}$ & $b$ & $Q$ & $n$ \\
\hline $500^{\circ} \mathrm{C}$ & $157000 \mathrm{MPa}$ & $135000 \mathrm{MPa}$ & $123000 \mathrm{MPa}$ & $4000 \mathrm{MPa}$ & 100000 & 850 & 1 & 3 & $-80 \mathrm{MPa}$ & $701 \mathrm{MPa}$ & 10.5 \\
$550^{\circ} \mathrm{C}$ & $155000 \mathrm{MPa}$ & $135000 \mathrm{MPa}$ & $107000 \mathrm{MPa}$ & $6000 \mathrm{MPa}$ & 100000 & 1000 & 1 & 4 & $-100 \mathrm{MPa}$ & $1265 \mathrm{MPa}$ & 6 \\
$600^{\circ} \mathrm{C}$ & $150000 \mathrm{MPa}$ & $135000 \mathrm{MPa}$ & $61000 \mathrm{MPa}$ & $11000 \mathrm{MPa}$ & 50000 & 1100 & 1 & 5 & $-110 \mathrm{MPa}$ & $3075 \mathrm{MPa}$ & 3.6 \\
\hline
\end{tabular}

Table 4.1: Material parameters provided in Koo and Kwon [41] for Gr. 91. Poisson's ratio was not given in the work as the implementation was uniaxial. The implementation here assumes $\nu=0.3$.

while the backstresses evolve with the Chaboche form

$$
\dot{\mathbf{X}}_{1}=-\left(\frac{2}{3} C_{i} \mathbf{n}+\sqrt{\frac{2}{3}} \gamma_{i} \mathbf{X}_{i}\right) y .
$$

The plastic strain rate is also a standard Chaboche form

$$
y=\sqrt{\frac{3}{2}}\left\langle\frac{f}{\sqrt{2 / 3} \eta}\right\rangle^{n} .
$$

The paper provides the values of these parameters for $T=500^{\circ} \mathrm{C}, T=550^{\circ} \mathrm{C}$, and $T=600^{\circ} \mathrm{C}$, listed in Table 4.1. Figure $4.1 \mathrm{a}$, b, and c provides verification the implementation matches the results from [41] . These three subfigures plot the hysteresis loops generated by strain-controlled cyclic cycling of the material model at a) $500^{\circ} \mathrm{C}$, b) $550^{\circ} \mathrm{C}$, and c) $600^{\circ} \mathrm{C}$ overlayed with model results reported in the paper.

A model could reasonably interpolate the parameters provided by Koo and Kwon [41] within the range $500^{\circ}$ to $600^{\circ} \mathrm{C}$ but not outside this range. A full assessment of the model versus the complete data set described above requires simulations at a large range of activation energies spanning from $g=0.1$ to $g=0.8$ as well as specific simulations at $550^{\circ} \mathrm{C}$. Varying $\dot{\varepsilon}$ from $10^{-6} \mathrm{~s}^{-1}$ to $1 \mathrm{~s}^{-1}$ at the temperatures with provided parameters produces a range of the activation energy spanning from 0.29 to 0.51 - sufficient to interrogate a reasonable range of unaxial properties. At creep strain rates of $10^{-9} \mathrm{~s}^{-1}$ this range decreases to 0.60 , so the comparison plots interrogate a good portion of the activation energy range spanned by the experimental data.

Figure $4.2 \mathrm{a}$ and b compares the Koo \& Kwon model to the uniaxial tension test data experimental data. Figure 4.2 (a) compares the values of the yield stress and (b) compares the hardening slope - this model shows approximately linear hardening in the strain regime of a uniaxial tension test. The model does not saturate in a reasonable period of time in uniaxial tension and so does not compare well to the saturation stress data. These curves plot three lines for the Koo \& Kwon model, one for each temperature, and varying the strain rate $\dot{\varepsilon}$ provides the variation of the normalized activation energy.

Figure 4.3 compares cyclic softening data between the model and the experimental database. Again, three lines show the results each parameter set $\left(T=500^{\circ} \mathrm{C}, T=550^{\circ} \mathrm{C}\right.$, and $T=600^{\circ} \mathrm{C}$ ), varying the strain rate to cause variation in the normalized activation energy.

Figure 4.4 summarizes the response of the model to stress-controlled cyclic loading, including the effect of a stress hold at the maximum tensile stress. Here the subfigures only 
FY17 Status Report on the Initial Development of a Constitutive Model for Grade 91 Steel July 2017
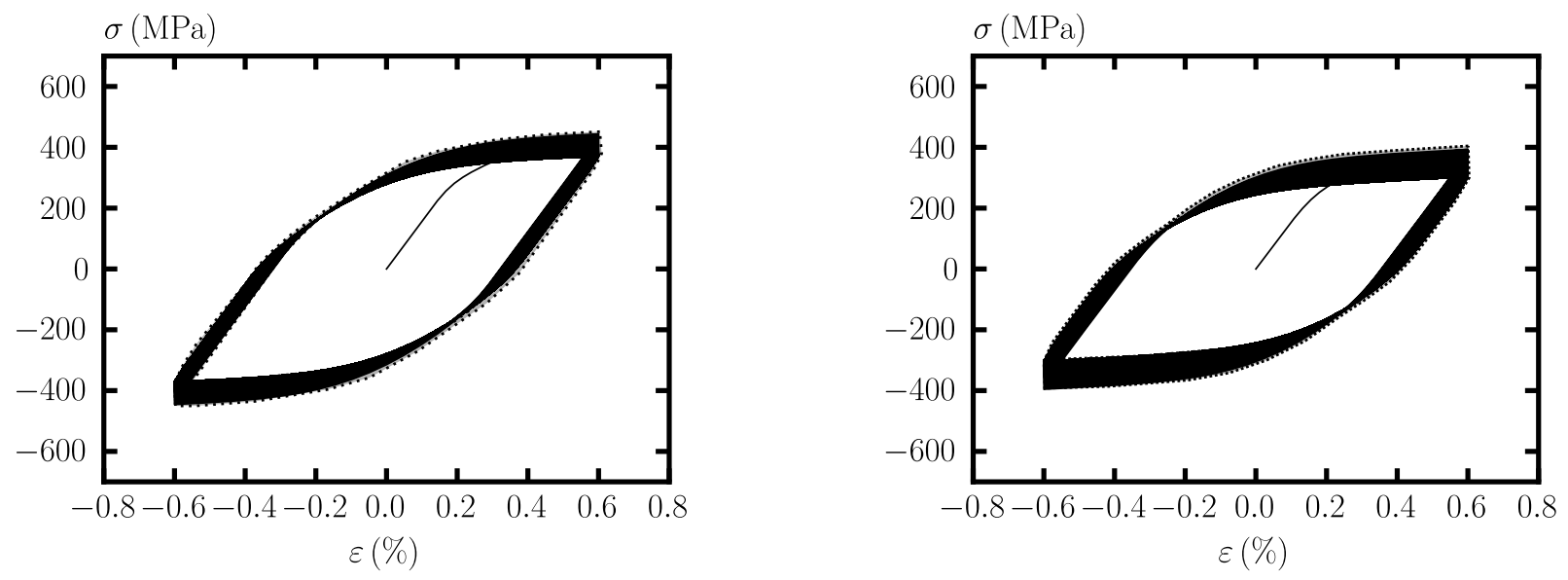

a)

b)

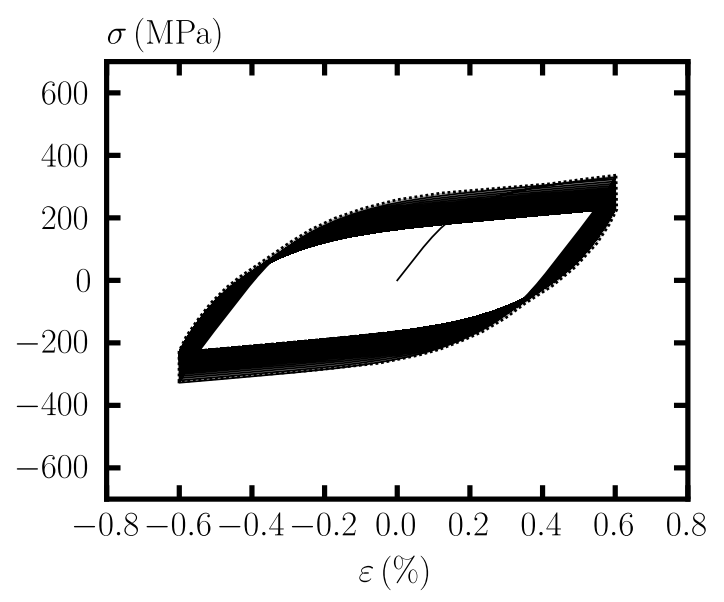

a)

Figure 4.1: Verification of the implementation of the Koo \& Kwon model. Plots compare results from the implemented model (black lines) for strain controlled cyclic deformation at $\dot{\varepsilon}=10^{-4} \mathrm{~s}^{-1}$ in the strain range $\pm 0.6 \%$ to results from Koo and Kwon [41] (dashed lines, filled region) at a) $500^{\circ} \mathrm{C}$, b) $550^{\circ} \mathrm{C}$, and c) $600^{\circ} \mathrm{C}$. 


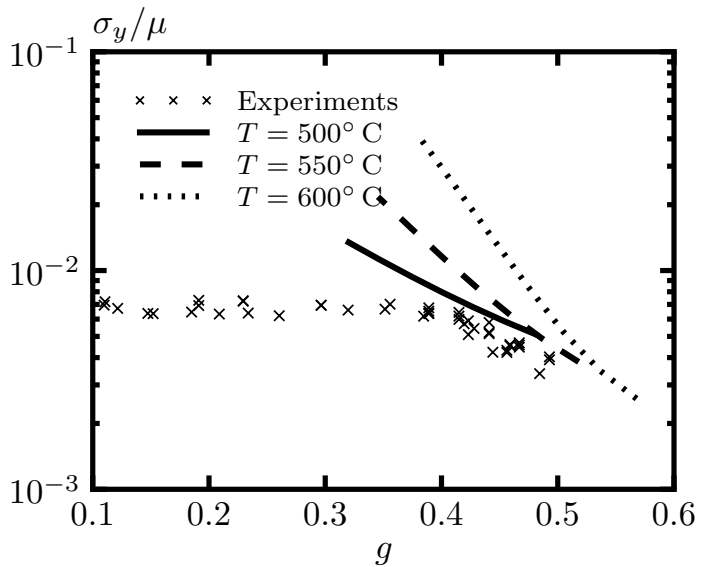

a)

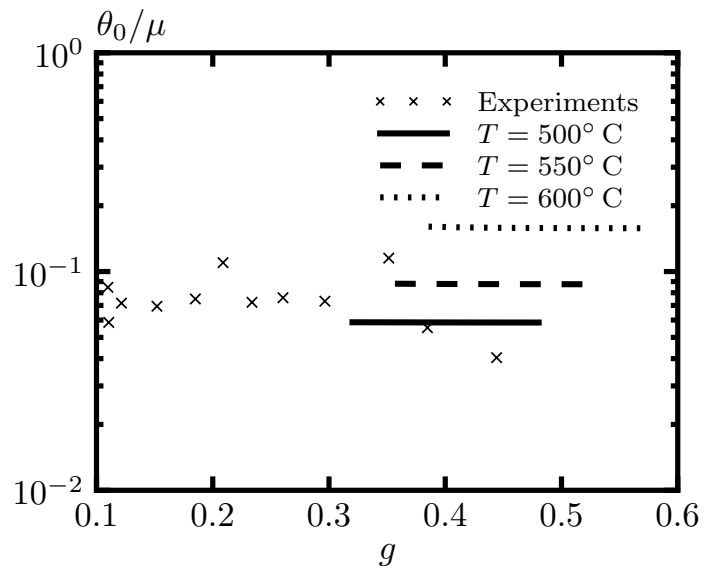

b)

Figure 4.2: Comparison of Koo \& Kwon model to uniaxial tension experimental data: a) yield stresses and b) initial hardening slope.

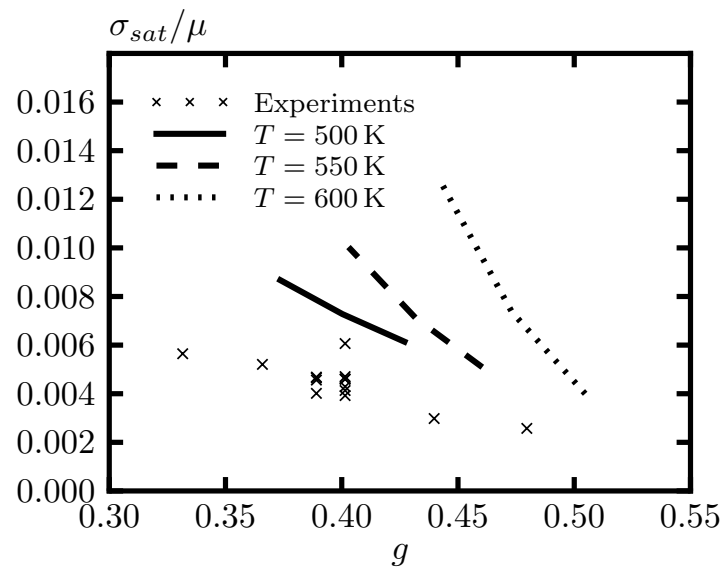

Figure 4.3: Comparison of Koo \& Kwon model to strain controlled cyclic softening tests. 


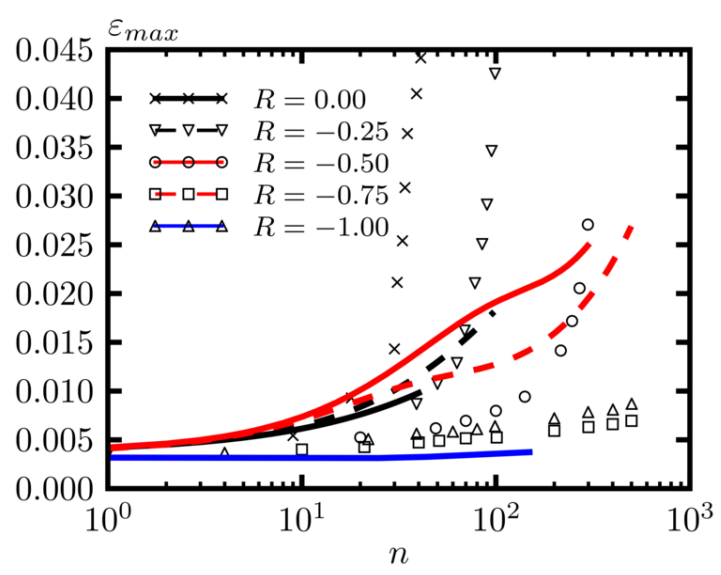

a)

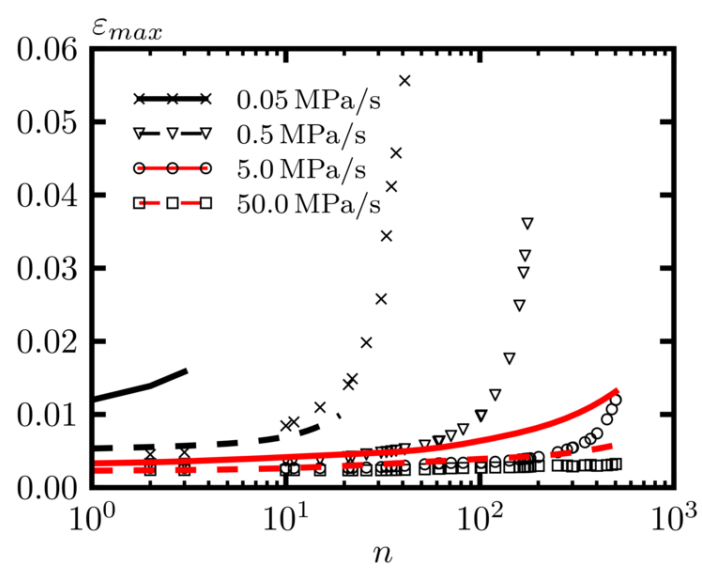

b)

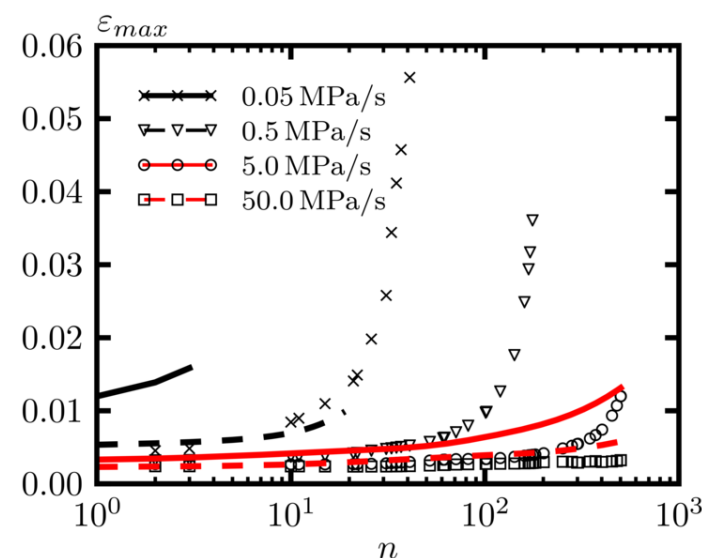

c)

Figure 4.4: Comparison between Koo \& Kwon model and experiments for stress-controlled cyclic loading. a) Effect of load ratio $R$, b) effect of loading rate $\dot{\sigma}$, and c) effect of hold time (hold at maximum tensile stress).

show only results for the parameter set at the test temperature of $T=550^{\circ} \mathrm{C}$. Some of the simulations halted before reaching the experimental number of cycles due to numerical instability in the model.

Finally, Fig. 4.5 summarizes the primary creep (a) and minimum creep rates (b) predicted by the model as a function of normalized activation energy. Here again there are three lines, one for each of the temperatures for which Koo and Kwon [41] provide calibrated model parameters. The lines on the plot, one for each temperature, correspond to a series of creep simulations at different loads, from 0.25 to $1.0 \sigma_{y}$.

The Koo \& Kwon model does not represent tertiary creep. Under creep deformation it starts deforming at some primary creep rate and then the creep strain rate decays exponentially to a constant, secondary rate. 


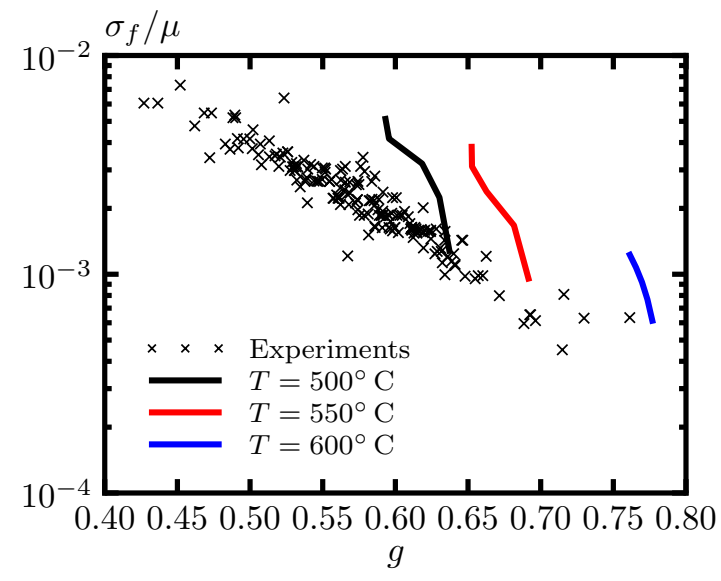

a)

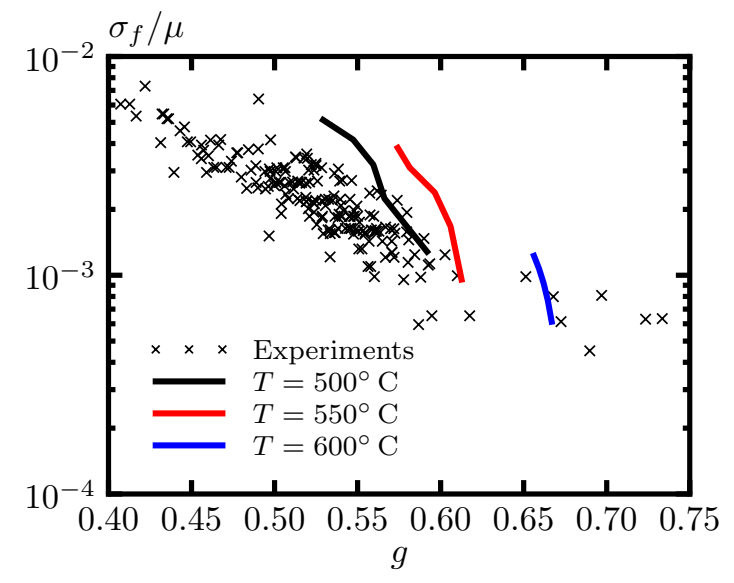

b)

Figure 4.5: Kocks-Mecking plots of the minimum creep rate (a) and primary creep rate (b) comparing the Koo \& Kwon model to the experiments.

\subsection{Yaguchi \& Takahashi model}

Reference [66] describes the formulation of the Yaguchi \& Takahashi model in 1D. They later extended the model to three dimension in $[67,68]$. This model has a modified Chaboche form with two backstresses, including the static recovery terms. Unlike Koo \& Kwon, who assigned cyclic hardening to the isotropic hardening parameter, Yaguchi \& Takahashi apply softening in the kinematic hardening. They use the isotropic hardening parameter to represent strain aging effects. The equations:

$$
\begin{gathered}
\dot{\varepsilon}_{p}=\mathbf{n} \dot{p} \\
\mathbf{n}=\frac{3}{2} \frac{\boldsymbol{\sigma}^{\prime}-\mathbf{X}^{\prime}}{J_{2}\left(\boldsymbol{\sigma}^{\prime}-\mathbf{X}^{\prime}\right)} \\
J_{2}\left(\mathbf{Y}^{\prime}\right)=\sqrt{\frac{3}{2} \mathbf{Y}^{\prime}: \mathbf{Y}^{\prime}} \\
\dot{p}=\left\langle\frac{J_{2}\left(\boldsymbol{\sigma}^{\prime}-\mathbf{X}^{\prime}\right)-\sigma_{a}}{D}\right\rangle^{n} \\
\dot{\mathbf{X}}_{1}=C_{1}\left(\frac{2}{3}\left(a_{10}-Q\right) \mathbf{n}-\mathbf{X}_{1}\right) \dot{p}-\gamma_{1} J_{2}\left(\mathbf{X}_{1}\right)^{m-1} \mathbf{X}_{1} \\
\dot{\mathbf{X}}_{2}=C_{2}\left(\frac{2}{3} a_{2} \mathbf{n}-\mathbf{X}_{2}\right) \dot{p}-\gamma_{2} J_{2}\left(\mathbf{X}_{2}\right)^{m-1} \mathbf{X}_{2} \\
\dot{Q}=d(q-Q) \dot{p}
\end{gathered}
$$




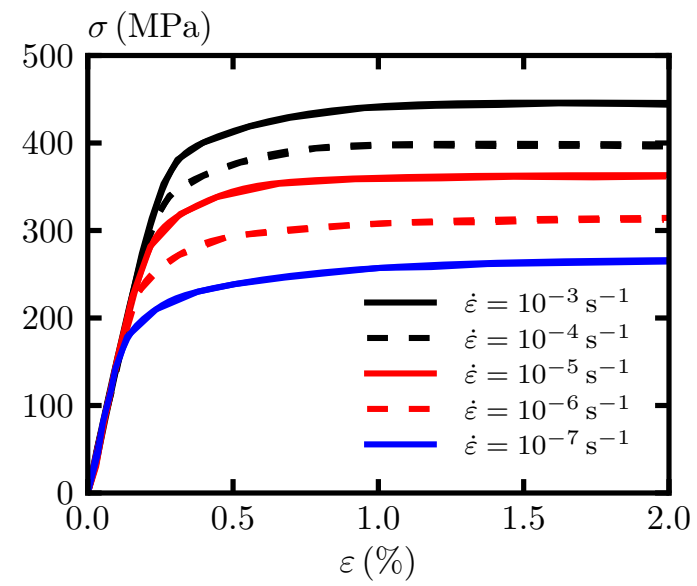

a)

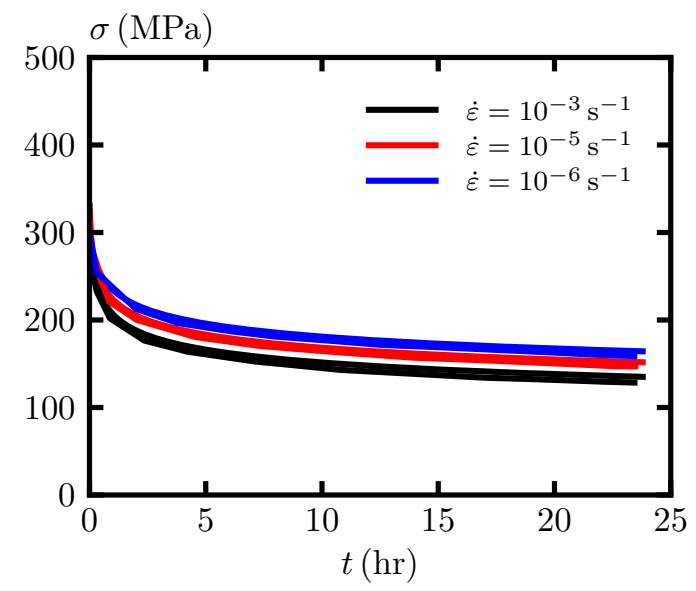

b)

Figure 4.6: Plots verifying the implementation of the Yaguchi \& Takahashi model. a) Simulations of uniaxial tension tests at various strain rates. b) Simulations of stress relaxation tests, with initial loading performed at various strain rates. The current implementation exactly matches the results provided in [66] and so the curves fall directly on top of each other.

$$
\begin{aligned}
\dot{\sigma}_{a} & =b\left(\sigma_{a s}-\sigma_{a}\right) \dot{p} \\
b & = \begin{cases}b_{h} & \sigma_{a s}-\sigma_{a} \geq 0 \\
b_{r} & \sigma_{a s}-\sigma_{a}<0\end{cases} \\
\sigma_{a s} & =\left\langle A+B \log _{10} \dot{p}\right\rangle
\end{aligned}
$$

describe the model. The internal variable database is $\mathbf{q}=\left[\begin{array}{llll}\mathbf{X}_{1} & \mathbf{X}_{2} & Q & \sigma_{a}\end{array}\right]$ with the evolution of $Q$ causing cyclic softening by changing the value of $a_{1}$ in the first Chaboche backstress evoluation equation.

In [66] Yaguchi \& Takahashi provide temperature continuous interpolation of all the material constants in the range $200^{\circ} \mathrm{C}<T<600^{\circ} \mathrm{C}$. This report omits the interpolation formulas, which are described in the original reference. In general they are empirical cubic polynomials or exponential formulas. [66] also provides the details of a non-isothermal implementation of the model and evidence that it successfully represents the results of strain rate and temperature jump tests. Figure 4.6 a and b verify the implementation here matches the model described in Yaguchi \& Takahashi's publications. These figures are (a) tension test simulations at increasing strain rates and (b) stress relaxation simulations, initially loading the model at different strain rates, both comparing data from [66] to the model implementation. These verification simulations are isothermal at $T=550^{\circ} \mathrm{C}$.

Yaguchi \& Takahashi provide continuous interpolation for the model parameters, so data spanning a range of normalized activation energies can be generated by varying the temperature, the strain rate, or both. The uniaxial tension and cyclic softening results below vary the activation energy by computing simulation results for a grid of temperature and strain rates defined by the Cartesian product of $T=\{473,523,573,623,673,723,773,823,873\} \mathrm{K}$ and $\dot{\varepsilon}=\left\{10^{-6}, 10^{-5}, 10^{-4}, 10^{-3}, 10^{-2}, 10^{-1}, 10^{0}\right\} \mathrm{s}^{-1}$. Creep simulations using the same pro- 


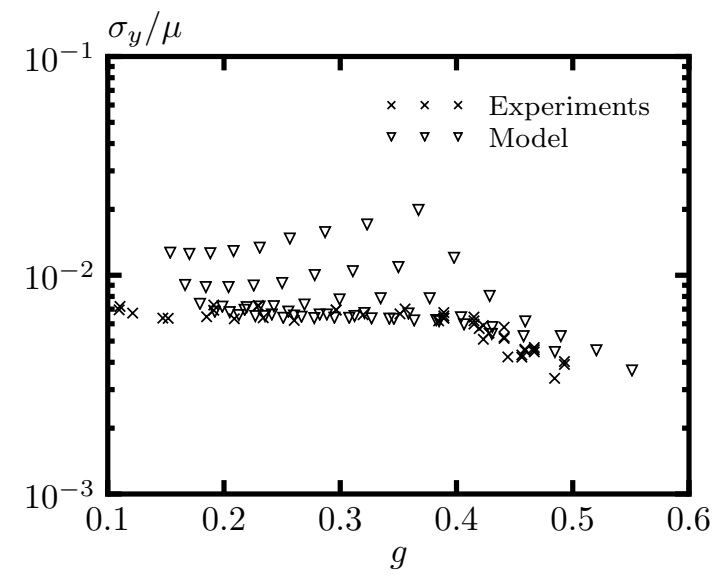

a)

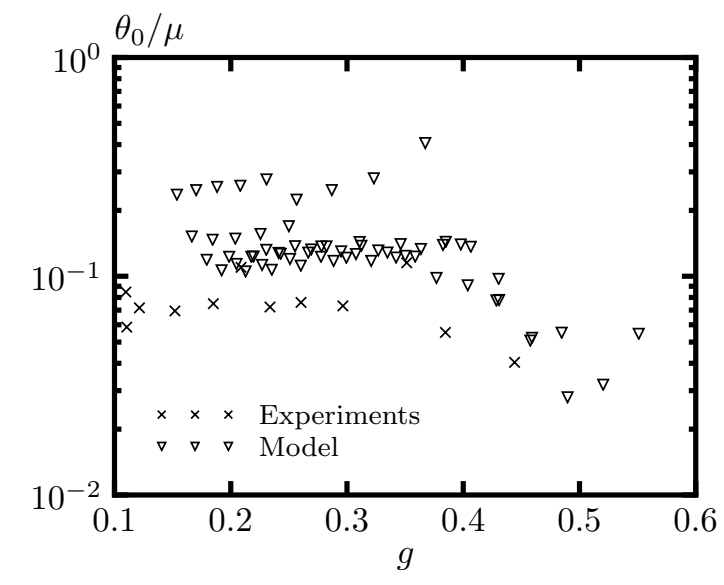

b)

Figure 4.7: Comparison of Yaguchi \& Takahashi model to uniaxial tension experimental data: a) yield stresses and b) initial hardening slope.

cedure as for the Koo \& Kwon model, described above, produces a range of normalized activation energies for $g=0.18$ to $g=0.78$. Unlike the experimental data in general the simulation data points do not fall along a single curve. Therefore, they are presented as points rather than a continuous line in the plots.

Figure 4.7, 4.8, 4.9, and 4.10 compare the Yaguchi \& Takahashi model to the experimental data. These plots correspond to Figs. 4.2-4.5 described above for the Koo \& Kwon model, with the only difference being the plots present a single curve for the Yaguchi \& Takahashi model, spanning activation energies with the procedure described above. The yield stresses are calculated with a $0.2 \%$ offset, with the initial hardening rate defined as the slope of the hardening curve at at $0.4 \%$ offset. As with the Koo \& Kwon model, in creep conditions the Yaguchi \& Takahashi saturates to a constant secondary creep rate and does not represent tertiary creep behavior.

\subsection{Model performance}

For monotonic or cyclic loading at given values of constant temperature and strain rate and for creep deformation at constant stress and temperature both models qualitatively have the desired response. Monotonically the models yield, develop some small amount of work hardening, and then soften. Increasing the temperature suppresses the period of initial hardening - at high temperatures the models only soften. Both models have saturating work softening under strain controlled cyclic loading described by exponential decay from an initial value of flow stress to some terminal, softened flow stress. Under stress controlled cyclic loading both models obey the general trends of 1) ratcheting rates increase as load cycles become less symmetric, 2) increasing the hold time at peak tensile load increases the amount of ratcheting, and 3) increasing the applied loading rate decreases the ratcheting strain. In creep, both models begin deforming at an initial primary creep rate and the deformation rate decreases exponentially to some terminal secondary creep rate. 


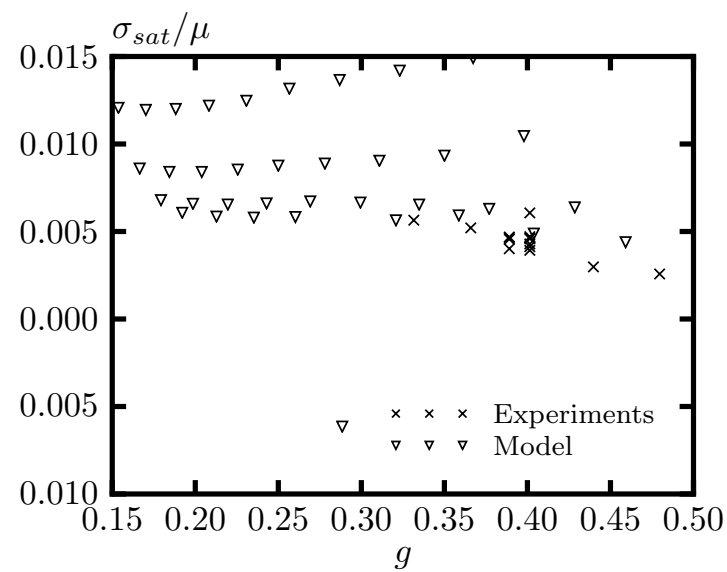

Figure 4.8: Comparison of Yaguchi \& Takahashi model to strain controlled cyclic softening tests.

Inaccuracy in the models falls into two general categories: qualitative discrepancy between the response at a given temperature and strain rate and temperature scaling schemes that are incompatible with the hypothesis that temperature and strain rate effects are linked via the Kock-Mecking normalized activation energy.

While qualitatively both models follow the trends of the experimental data for each type of experiment, quantitatively neither model is accurate across all tested temperatures and strain rates. In general, the Yaguchi \& Takahashi is more accurate than the Koo \& Kwon model, which tends to overestimate the flow stress at all temperatures and strain rates. This overestimation causes errors when comparing to monotonic tension tests but also affects the remainder of the tests by providing an incorrect initial point for cyclic softening, ratcheting, and creep simulations. The Koo \& Kwon also fails to quantitatively predict the evolution of ratcheting strain with the number of cycles in the stress controlled cyclic simulations, though it does capture the correct trends. At higher temperatures this model also underpredicts the amount of cyclic softening, leading to a higher softened flow stress than those observed in the experiments. This model also tends to unconservatively underestimate the ratcheting strain per cycle in stress controlled cyclic simulations. Primary and minimum creep rates are close to the experimental values and the model conservatively overestimates both deformation rates.

The Yaguchi \& Takahashi suffers from similar quantitative errors. It overestimates the monotonic yield stress at higher strain rates but quite accurately recovers the yield stress at low rates. Similarly the model overestimates the saturated, softened flow stress for cyclic deformation at higher strain rates. This model conservatively overpredicts the amount of ratcheting strain per cycle in stress controlled fatigue simulations and furthermore accurately models the effect of hold time and stress rate on ratcheting strain. However, it greatly overestimates the ratcheting strain for highly non-symmetric load cycles. Unfortunately, for low applied loads, the Yaguchi \& Takahashi model can greatly underestimate the primary and minimum creep rates.

Beyond quantitative inaccuracy at fixed temperatures the response of both models does 


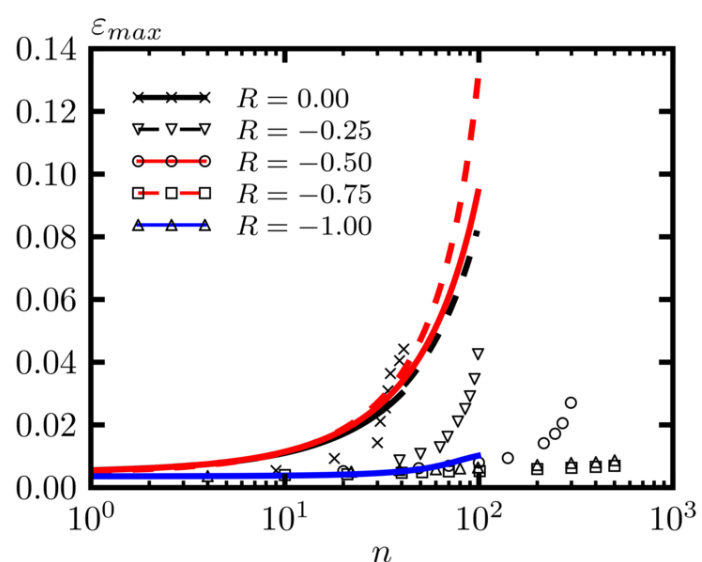

a)

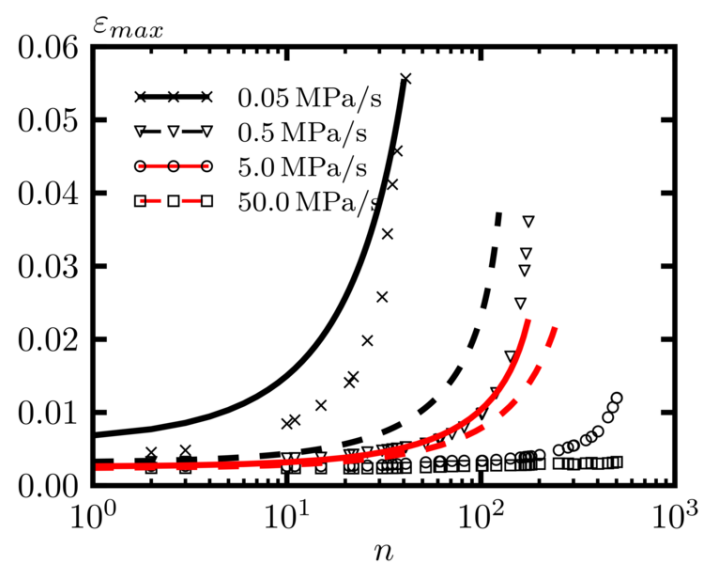

b)

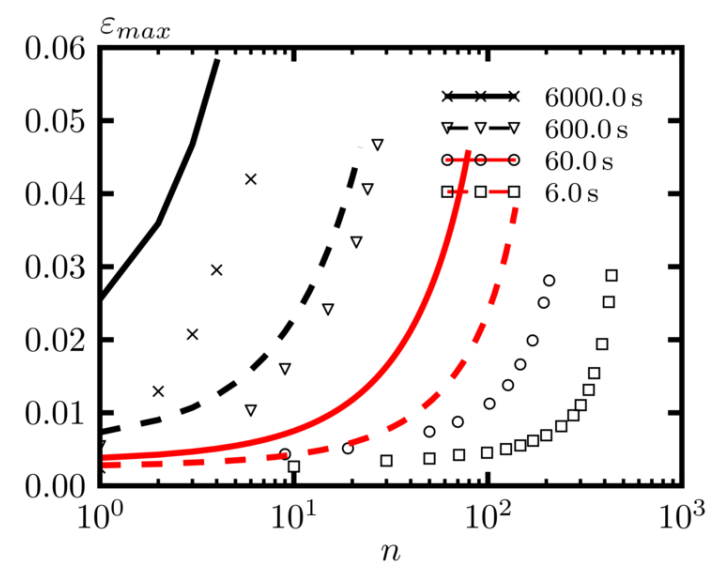

c)

Figure 4.9: Comparison between Yaguchi \& Takahashi model and experiments for stresscontrolled cyclic loading. a) Effect of load ratio $R, \mathrm{~b}$ ) effect of loading rate $\dot{\sigma}$, c) effect of hold time (hold at max tensile stress). 


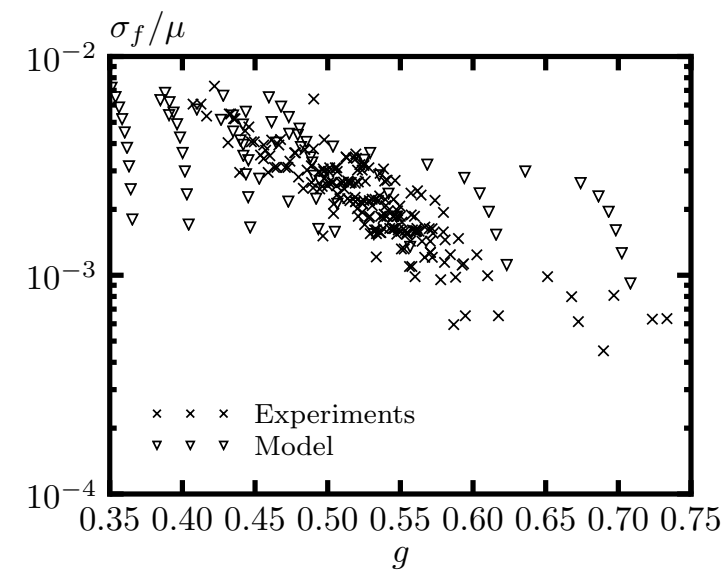

a)

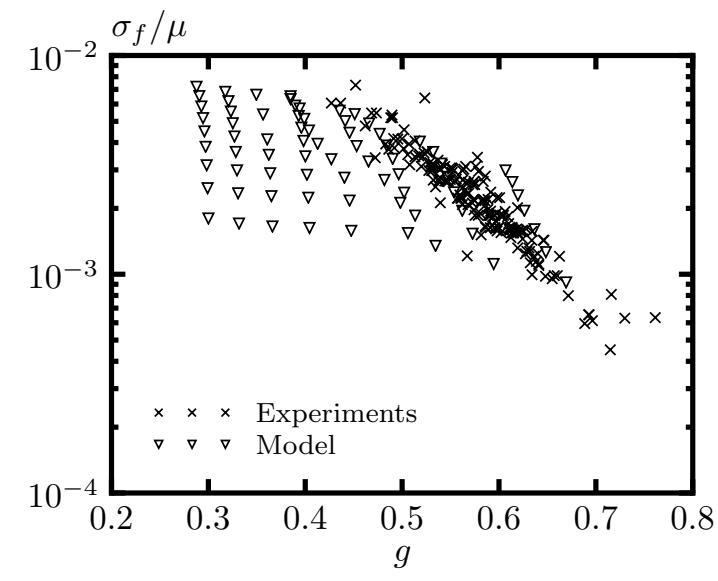

b)

Figure 4.10: Kocks-Mecking plots of the minimum creep rate (a) and primary creep rate (b) comparing the Yaguchi \& Takahashi model to the experiments.

not correctly scale with temperature. While the experimental data for the yield stress, hardening slope, softened flow stress, primary creep rate, and secondary creep rate all fall along universal curves when plotted as functions of the Kocks-Mecking normalized activation energy the model response for these quantities does not. Either incorrect strain rate scaling, temperature scaling, or both could cause this problem. However, both models obey a Perzyna strain rate relation of the form

$$
\dot{\varepsilon} \sim\left\langle\frac{f(\boldsymbol{\sigma})}{D}\right\rangle^{n} .
$$

A relation between normalized activation energy and the logarithm of the stress implies a rate law of the type

$$
\dot{\varepsilon} \sim\left(\frac{f(\boldsymbol{\sigma})}{D}\right)^{\frac{\mu b^{3}}{k T}}
$$

which, for fixed $T$, is a Perzyna model. This explains why the model results fall onto continuous curves on normalized activation energy plots for fixed temperature and varying strain rate. However, the actual prediction of the Kocks-Mecking analysis of the experimental data is that these relations should be bilinear functions of the stress rather than the nonlinear relations demonstrated by the models.

A Kocks-Mecking model further predicts a particular temperature scaling relation that neither implemented model matches. The Koo \& Kwon model does not provide a temperature interpolation scheme and clearly the constants for each of the three provided temperature do not produce results falling along the master curve predicted by the Kocks-Mecking analysis. Similarly, the temperature interpolation scheme provided by the Yaguchi \& Takahashi formulation (generally cubic polynomials) also does not follow the predicted scaling from Kocks-Mecking analysis. This means that the model results for fixed strain rate but varying temperature do not fall along a single master curve.

However, each of the models approximately recovers an aspect of the Kocks-Mecking 
analysis of the experimental data. For each temperature, the Koo \& Kwon model produces approximately log-linear results on normalized activation energy plots. However, each temperature produces curves with different values of slope and intercept. Very roughly, the slopes of these lines match with the non-zero, rate dependent portion of the master curve found in Chapter 2.

While the Yaguchi \& Takahashi produces curves that are not log-linear it does approximately recreate the bifurcated behavior of the experimental master curve. The yield stress plot (Fig. 4.7) clearly illustrates this: the results have a clear discontinuity near $g=0.37$, which is near the rate-dependent/independent cutoff predicted by the analysis of the experimental data. 


\section{Modeling the rate sensitivity of advanced reactor structural ma- terials}

\subsection{Introduction}

Neither the Koo and Kwon nor Yaguhi and Takahashi models successfully recreate the KocksMecking master curve developed for Gr. 91 directly from the available experimental data. Therefore, neither model is accurately representing rate sensitivity in the material requiring the development of a new method to represent material rate sensitivity in a viscoplastic material model over the expected range of plant operating temperatures and strain rates.

In the regime where creep and plasticity can be decoupled the superposition of creep and plastic strains in a constitutive model is adequate for inelastic analysis. However, in the regime where creep and plastic deformations are coupled the ASME code requires the use of a unified viscoplastic model. The development of such models is ongoing for advanced reactor materials $[65,57,41]$. A complete inelastic material model used for plant design must also extend to the low temperature, rate independent regime to model plant shutdown and cooling transients. Extending a viscoplastic material model to an approximately rate insensitive response by using a large rate sensitivity exponent is an unsatisfying solution due to the well-known numerical instability caused by the high stiffness of the resulting ordinary differential equations [54].

This chapter proposes a model that can transition from a rate independent response at low temperatures and fast strain rates to a rate dependent response at lower, creep strain rates and high temperatures. This model incorporates the idea of a normalized thermal activation energy developed by Kocks, Mecking, and co-workers [39, 40, 49] and described in detail above. This activation energy describes the thermal energy available for a dislocation to use to overcome microscale obstacles. This energy increases with increasing temperature and decreases with increasing strain rate. Below some critical activation energy plastic flow is rate insensitive. Above this critical value thermally-activated mechanisms (e.g. climb or cross-slip) control the rate of deformation and plastic flow becomes rate sensitive.

As developed above for Gr. 91, the normalized activation energy concepts suggests a master curve plotting the normalized energy versus the flow stress, at fixed state, normalized by the temperature-dependent shear modulus [39]. Materials obeying the Kocks-Mecking theory typically have master curves consisting of straight line segments. A slope of zero indicates a rate-insensitive regime and a non-zero slope indicates rate sensitivity and provides the constitutive form describing the material rate sensitivity as a function of temperature. Chapter 2 developed this master curve for Gr. 91

This chapter proposes using a bilinear description of the master curve as a model for the material flow stress that can transition between the rate dependent and rate independent regimes. Previous researchers have addressed this problem by supplying a modified time step to a viscoplastic model to emulate a rate independent response [63, 64]. Other previous solutions include Bruhns and Rott [4] who included weighted rate dependent and independent contributions to the inelastic strain, using an additional internal variable to change the relative contributions of the two terms to transition between rate independent and dependent behavior. Freed and Walker [29] developed a model which selects either a rate dependent or independent step based on the current deviatoric stress. Fundamentally, this is the form 


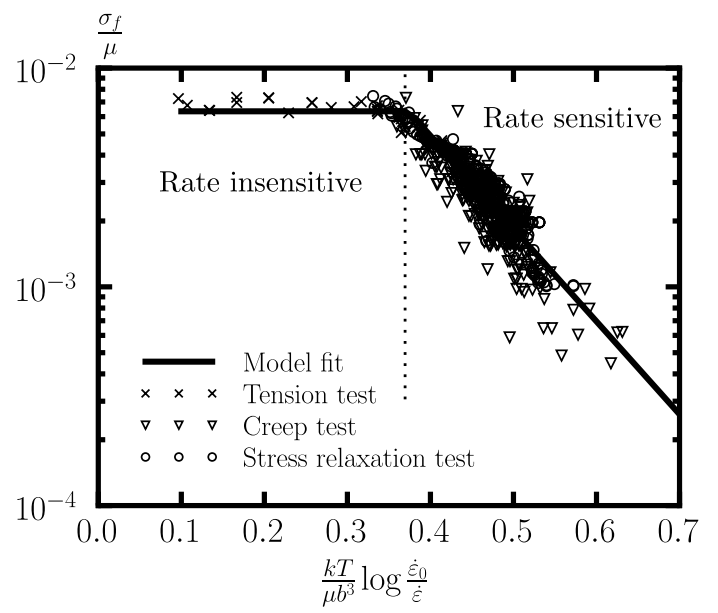

(a)

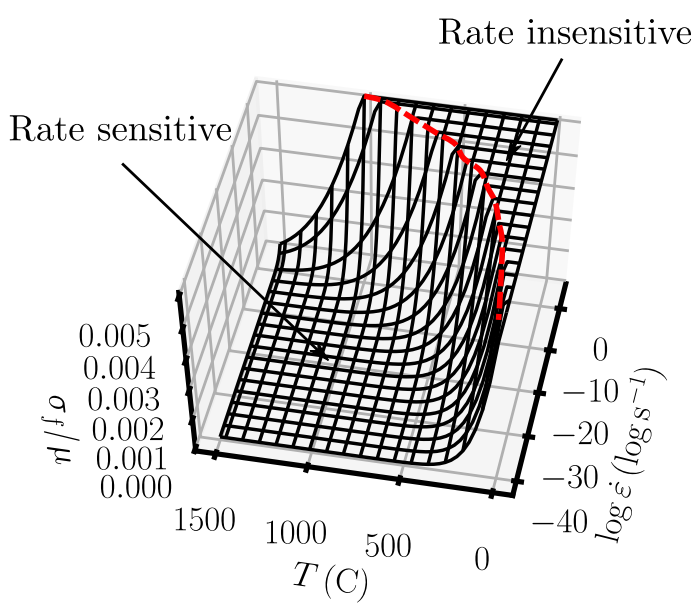

(b)

Figure 5.1: Rate sensitivity diagrams for Grade 91 steel. (a) Kocks-Mecking plot summarizing experimental data and establishing bilinear fit. (b) 3D plot illustrating the dependence of normalized flow stress on temperature and strain rate with the transition between rate sensitive and insensitive behavior labeled.

the current model adopts. However the cutoff stress described by Freed and Walker is not temperature dependent which, as shown below, does not agree with the known scaling of the rate independent flow stress with the temperature-dependent shear modulus.

The method for modeling rate sensitivity and switching between rate sensitive and insensitive steps developed here underlies the Grade 91 inelastic constitutive model developed in the next section. Essentially, a complete model supplements the model for rates sensitivity developed here with a model for material state evolution - flow stress hardening and softening. In general, and particularly in the coupled creep/plasticity regime, a successful model must first correctly represent the underlying material rate sensitivity before addressing hardening and soften behavior.

\subsection{Modeling the transition between rate sensitive and rate insensitive response}

Figure 5.1 reproduces the Kocks-Mecking master curve generated for Gr. 91 in the previous sections. The master curve shows a general trend: on the semilog scale an initial rate insensitive regime with approximately zero slope followed by a transition to rate dependent behavior at a critical activation energy with an approximately linear slope. The function

$$
\log \frac{\sigma_{f}}{\mu}= \begin{cases}C & g<g_{0} \\ A g+B & g \geq g_{0}\end{cases}
$$

represents this response. Only three of the the four parameters in this equation $-A, B, C$, and $g_{0}$ - are independent. Continuity of the two line segments determines the final constant. Table 5.1 lists the constants describing the bilinear model for Gr. 91 calculated by nonlinear least squares regression. 


\begin{tabular}{ccccc}
\hline Material & $g_{0}$ & $A$ & $B$ & $C$ \\
\hline Gr. 91 & 0.374 & -9.77 & -1.41 & -5.06 \\
\hline
\end{tabular}

Table 5.1: Bilinear descriptions of the master curve for Gr. 91.

The idea of a critical value of activation energy separating rate dependent deformation from rate independent deformation can be adapted into a model for the plastic deformation of a material that spans the transition from rate independent to rate dependent response. Consider the incremental interface a finite element solver typically uses to call a small deformation material model:

$$
\boldsymbol{\sigma}_{n+1}, \boldsymbol{h}_{n+1}=f\left(\Delta \varepsilon_{n+1}, \Delta t_{n+1}, T_{n+1}, \boldsymbol{h}_{n}\right) .
$$

A constitutive model, as defined by this interface, is a function that takes the strain increment and time increment over the load step, $\Delta \varepsilon_{n+1}$ and $\Delta t_{n+1}$, along with the temperature $T_{n+1}$ and the vector of history variables at the previous time step $\boldsymbol{h}_{n}$, and returns the stress and history variables at the next time step $\boldsymbol{\sigma}_{n+1}$ and $\boldsymbol{h}_{n+1}$. This interface obscures all details of the model and the integration strategy but can accommodate both rate independent and viscoplastic models. In this section consider two different material models described by functions $f_{1}$ and $f_{2}$. The first model, defined by $f_{1}$, is rate independent. This means that strictly its functional form is

$$
\boldsymbol{\sigma}_{n+1}, \boldsymbol{h}_{n+1}=f_{1}\left(\Delta \boldsymbol{\varepsilon}_{n+1}, T_{n+1}, \boldsymbol{h}_{n}\right)
$$

i.e. it does not depend on the time increment, only the strain increment. The second model, defined by $f_{2}$, is rate dependent.

Furthermore, assume that $f_{1}$ and $f_{2}$ have compatible hardening models. Two hardening models are compatible if they have the same vector of internal variables $\boldsymbol{h}$. With compatible hardening models, given the strain increment, time step, and temperature a composite model could call either the rate independent or rate dependent model to update the stress and history variables. A composite model accounting for the rate-independent to rate-dependent transition requires a method for selecting which type of step to take.

Consider more specific material updates. Let $f_{1}$ be rate independent perfect plasticity, solving the equations:

$$
\begin{array}{rlrl}
\boldsymbol{\sigma} & =\boldsymbol{C}:\left(\boldsymbol{\varepsilon}-\boldsymbol{\varepsilon}^{p}\right) \\
\dot{\boldsymbol{\varepsilon}}^{p} & = & \gamma \frac{\partial f}{\partial \boldsymbol{\sigma}} \\
\gamma & \geq & 0 \\
f\left(\boldsymbol{\sigma}, \sigma_{Y}\right) & \leq & 0 \\
\gamma f\left(\boldsymbol{\sigma}, \sigma_{Y}\right) & = & 0 \\
\gamma \dot{f}\left(\boldsymbol{\sigma}, \sigma_{Y}\right) & = & & 0
\end{array}
$$

for temperature-dependent elastic tensor $\boldsymbol{C}$ and a yield surface $f\left(\boldsymbol{\sigma}, \sigma_{Y}\right)$ controlled by a single parameter $\sigma_{Y}$, which can be a function of temperature. Let $f_{2}$ be classical perfect 
Perzyna viscoplasticity, integrating the equations

$$
\begin{aligned}
\boldsymbol{\sigma} & =\boldsymbol{C}: & \left(\boldsymbol{\varepsilon}-\boldsymbol{\varepsilon}^{p}\right) \\
\dot{\boldsymbol{\varepsilon}}^{p} & = & \gamma \frac{\partial f}{\partial \boldsymbol{\sigma}} \\
\gamma & = & \left\langle\frac{f^{n}}{\eta}\right\rangle
\end{aligned}
$$

again for elastic tensor $\boldsymbol{C}$, a yield surface $f\left(\boldsymbol{\sigma}, \sigma_{Y}\right)$ controlled by a single parameter $\sigma_{Y}$, and rate constants $n$ and $\eta$. All these constants can be temperature dependent. In Eq. 5.12 $\langle$ are the Macaulay brackets. Suppose these two models have the same elastic constants and yield/flow $f$ surface and suppose for uniaxial stress states $f\left(\boldsymbol{\sigma}, \sigma_{Y}\right)=\sigma-\sigma_{Y}$. The models have compatible hardening because the sets of history variables are the same: the empty set.

Given the bilinear description of the master curve described above in terms of the constants $g_{0}, A, B$, and $C$ the composite model takes the form

$$
\boldsymbol{\sigma}_{n+1}, \boldsymbol{h}_{n+1}=f_{\text {composite }}\left(\Delta \varepsilon_{n+1}, \Delta t_{n+1}, T_{n+1}, \boldsymbol{h}_{n}\right) .
$$

The model evaluates the function $f_{\text {composite }}$ as follows. First calculate the effective strain rate over the step

$$
\dot{\varepsilon}_{n+1}=\sqrt{\frac{2}{3}}\left\|\frac{\Delta \varepsilon_{n+1}}{\Delta t_{n+1}}\right\|
$$

and the normalized activation energy

$$
g_{n+1}=\frac{k T_{n+1}}{\mu_{n+1} b^{3}} \log \left(\frac{\dot{\varepsilon}_{0}}{\dot{\varepsilon}_{n+1}}\right)
$$

with $\mu_{n+1}$ the temperature dependent shear modulus corresponding to the step temperature $T_{n+1}$. Then if $g \leq g_{0}$ take a rate independent step by setting

$$
\sigma_{Y}=\mu_{n+1} e^{C}
$$

and solve Eqs. 5.4-5.9 to update to $\boldsymbol{\sigma}_{n+1}$. If $g>g_{0}$ take a rate dependent step by setting

$$
\begin{aligned}
& \sigma_{Y}=\quad 0 \\
& n=\quad-\frac{\mu b^{3}}{k T A} \\
& \eta=e^{B} \mu \dot{\varepsilon}_{0}^{k T A /\left(\mu b^{3}\right)}
\end{aligned}
$$

and integrating Eqs. 5.10-5.12 to find the new stress tensor. Equations 5.18 and 5.19 are an algebraic rearrangement of the linear part of the bilinear model of the master curves:

$$
\begin{array}{rlr}
\log \frac{\sigma_{f}}{\mu} & = & A g+B \\
\frac{\sigma_{f}}{\mu e^{B}} & =\left(\frac{\dot{\varepsilon}}{\dot{\varepsilon}_{0}}\right)^{-A k T /\left(\mu b^{3}\right)} \\
\dot{\varepsilon} & =\dot{\varepsilon}_{0}\left(\frac{\sigma_{f}}{\mu e^{B}}\right)^{-\mu b^{3} /(A k T)} .
\end{array}
$$




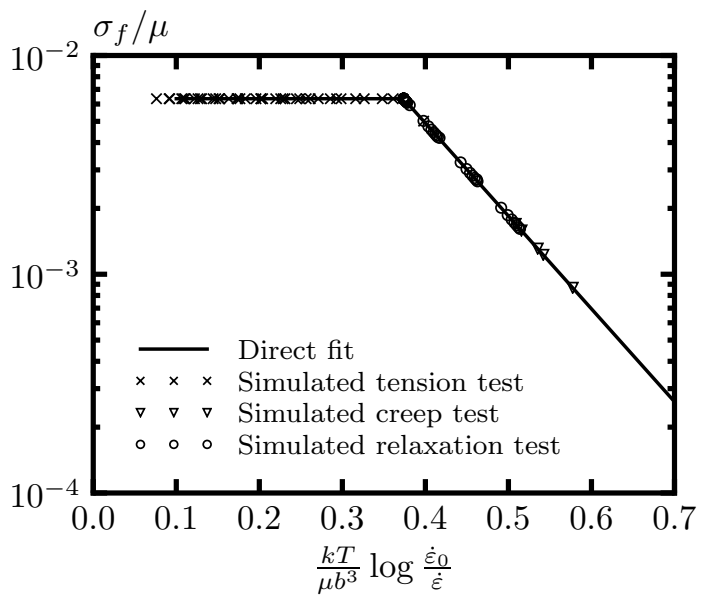

Figure 5.2: This plot compares a series of simulated tests using the model described here for Gr. 91 to the direct bilinear fit to the experimental data. The rate sensitivity of the composite model causes all the simulated test data, for monotonic tension, creep, and stress relaxation tests, to fall directly on the best fit line describing the experimental data.

The temperature dependent parameters $n$ and $\eta$ can be identified from this final expression.

Figure 5.2 shows results using this composite model for Gr. 91 rate sensitivity with the constants identified in Table 5.1 and the temperature dependent elastic properties from the ASME code. This figure represent a series of simulations: uniaxial tension at various temperatures and strain rates, creep at various temperatures and applied loads, and stress relaxation at a variety of temperatures and initial strains. The figure then plots the results of these simulations on a Kocks-Mecking diagram, extracting the activation energy and flow stress using the procedures developed above and overlays the bilinear description of the master curve calibrated directly to the experimental The simulation results reproduce the best-fit bilinear master curve meaning the composite model developed here exactly reproduces the average rate sensitivity of Gr. 91 .

\subsection{Discussion}

\subsubsection{Plasticity mechanisms}

The master rate sensitivity plots developed for Gr. 91 shows two types of behavior: a rateinsensitive regime at high strain rates and low temperatures and a rate sensitive regime at low strain rates and high temperatures. These two regimes relate to different microscale behavior.

The master curve developed here attempts to compare different experiments on Gr. 91 at fixed material state so the diagrams do not consider microstructure evolution. Dislocations mediate plasticity in Gr. 91. At fixed state the rate sensitivity of the material will be controlled by two processes: 1) dislocations moving between microstructural obstacles and 2) the process by which a dislocation overcomes an obstacle to continue its motion.

First consider the case of moderate strain rates where the average dislocation velocity 
is much faster than the rate of macroscale deformation. In this regime dislocations move between obstacles nearly instantaneously and the process of overcoming obstacles sets the material rate sensitivity. Here distinguish two types of behavior. First, with sufficient time and thermal energy, a dislocation can overcome an obstacle with some thermally activated process, for example dislocation climb or cross slip. Secondly, as additional dislocation lines slip into the obstacle a pile up develops, increasing the stress field pushing the pinned dislocations past the obstacle. Eventually the pile up will accumulate enough stress to push a dislocation through the obstacle and continue its motion.

In actuality both mechanisms occur simultaneously and the stress of a pile up lowers the energy barrier overcome with a thermal process. We assume dislocation move between obstacles instantly so each obstacle will have some average number of dislocations piled up, providing a force lowering the effective energy barrier of the obstacle. The average number of dislocations in a pile up depends only on the structure of the material and not any kinetic factors. In an average sense the flow stress in the material is equal to the stress required to lower the energy barrier sufficiently to allow plastic flow at a given strain rate. At fast strain rates and/or low temperatures there is not enough time for random atomic motion to help the dislocation move past the obstacle. The flow stress, aided by the stress induced by pileups, must be sufficient to force dislocations past obstacles. This stress is the same regardless of the rate of deformation and is controlled entirely by the structure of the material. However, the stress at the tip of a pileup scales with the material shear modulus [34], which introduces a moderate temperature dependence. This mechanism controls plastic flow in the rate independent regime identified in the master curves.

As the temperature increases or the strain rate decreases thermal activation becomes significant in helping dislocations overcome obstacles. Decreasing the strain rate increases the time dislocations spend at obstacles, increasing the chances that random atomic vibration will move a dislocation past an obstacle. Similarly, as the temperature increases the magnitude of random vibration increases likewise increasing the chance a dislocation will move over an obstacle. These thermally activated mechanisms decrease the average flow stress required to maintain a given deformation rate. In this regime both temperature and strain rate significantly affect the flow stress. This is the rate dependent region identified above at higher normalized activation energies.

\subsubsection{Work hardening}

The previous examples had either no work hardening or simple linear isotropic hardening with a low work hardening rate. Figure 5.3 demonstrates the composite model works with more sophisticated isotropic hardening schemes. This simulation of a strain rate jump test uses the composite model for strain rate sensitivity developed above supplemented with Voce isotropic hardening. The figure shows both the stress/strain curve and the work hardening curve.

The work hardening curve illustrates how the composite model carries the evolution of the history variables across strain rate jumps. After a transient, in the rate sensitive regime, the work hardening curve is continuous across strain rates. This means that the work hardening rate is continuous across a strain rate jump. The composite modeling approach described here easily extends to more sophisticated models of combined isotropic and kinematic hard- 


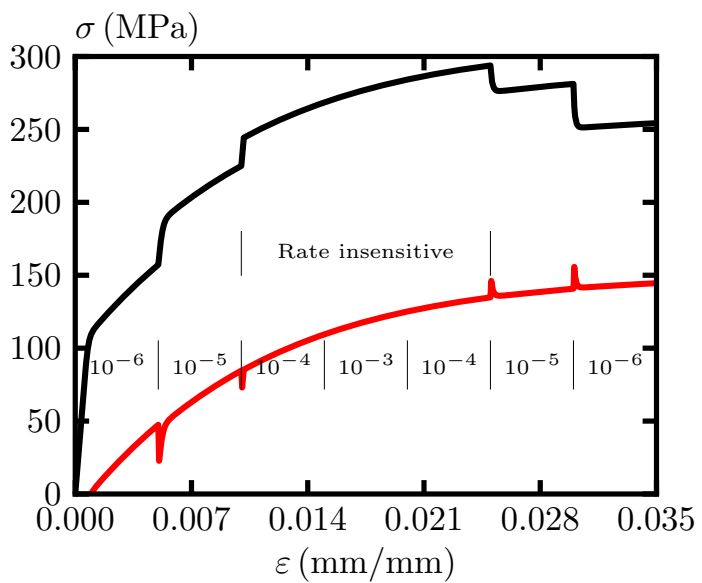

Figure 5.3: Simulation using the calibrated strain rate sensitivity model at $750^{\circ} \mathrm{C}$ and Voce isotropic hardening. In the figure the black line plots the flow stress $\sigma_{f}$ and the red line plots the difference $\sigma_{f}-\sigma_{y}$ with $\sigma_{y}$ the yield stress at the current strain rate. This simulation demonstrates the continuation of work hardening across the rate sensitive/insensitive boundary and the ability of the model to transition from rate sensitive to rate insensitive behavior and subsequently back to rate sensitive deformation. The labeled strain rates have units of $s^{-1}$.

ening provided the hardening model can be implemented in either a rate dependent or rate independent form. Most common empirical hardening models meet this criteria [10].

\subsubsection{Numerical performance}

The composite model essentially merges two separate material models into a framework for dealing with rate sensitivity. The numerical performance of each of the two sub-models, i.e. the rate dependent and rate independent steps, depends on the integration strategies and other implementation details and the performance of the sub-models can be tuned independently from the overall composite modeling framework. However, one aspect of numerical stability directly related to the composite model is the convergence of the global nonlinear equilibrium equations when a load step spans the transition between rate independent and rate dependent behavior. In addition to the updated stress and history variables a finite element method that implements solving the nonlinear incremental equilibrium equation with a full Newton scheme also requires a material model return the algorithmic tangent

$$
\boldsymbol{A}_{n+1}=\frac{d \boldsymbol{\sigma}_{n+1}}{d \Delta \varepsilon_{n+1}}
$$

These algorithmic tangents are integrated over the elements and assembled into the global Jacobian matrix used to solve the nonlinear equilibrium equations with Newton iteration. Here we assume that each of the two sub-models returns the correct algorithmic tangent and the composite model simply returns tangent corresponding to the type of step. This strategy ensures the overall Jacobian remains consistent with the material updates. However, 


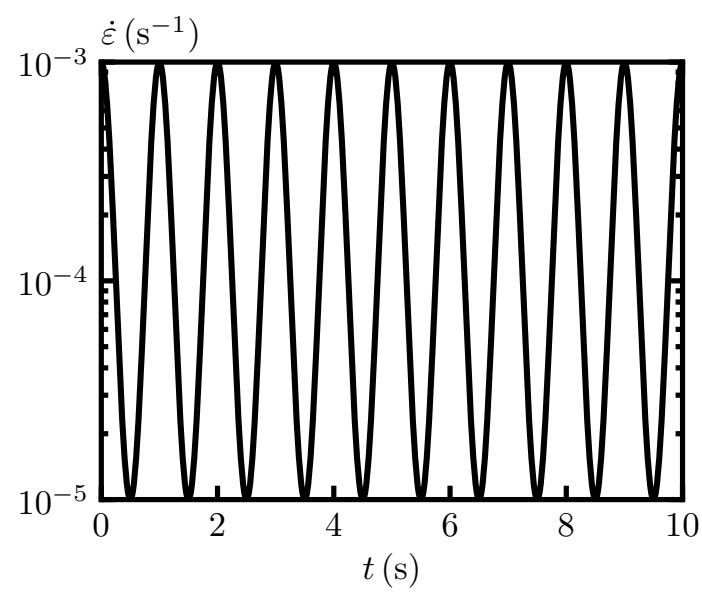

(a)

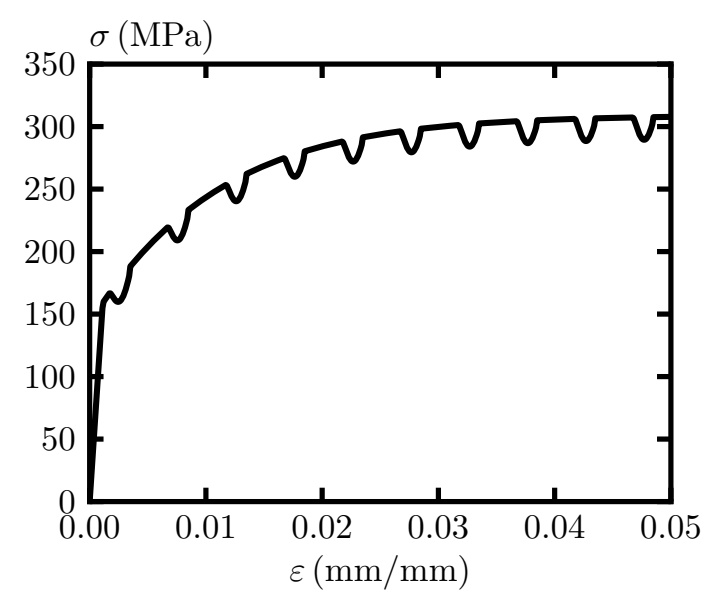

(b)

Figure 5.4: Example simulation of a load history involving rapid strain rate oscillations. (a) The strain rate history imposed on the model. (b) The resulting stress/strain curve.

the jump between a rate independent and rate dependent step could affect the continuity of the equilibrium equations, affecting the convergence of the overall Newton scheme.

Figure 5.4 shows results from a simulation designed to test the numerical performance of this transition between rate independent and rate dependent steps. This is a simulation of a uniaxial test with applied strain rates given by the cosine wave shown in Fig. 5.4a. These strain rates cause the simulation to oscillate between rate dependent and rate independent behavior. The material model is the same one used to generate Fig. 5.3. Figure 5.4b shows the resulting stress/strain hysteresis loop. Newton's method converged quadratically at each load step demonstrating the composite model did not affect the underlying quadratic convergence of the rate independent and rate dependent sub-models.

This is a simple example. Good convergence with the composite model may require additional continuity constraints on the underlying material models that have not been uncovered in this work. The numerical stability of the composite method will be a topic of future research.

\subsection{Conclusions}

The key results of this chapter are:

- A composite model for material rate sensitivity that can span across rate dependent and independent regimes. This composite framework can form the basis for models used for inelastic design of advanced reactor concepts because it can span the full range of plant operating temperatures, from shutdown to high temperature transients.

- The parameters for the strain rate sensitivity for Grade 91, calibrated to the experimental database. 
Combined with a model for work hardening/softening the composite modeling approach allows for a complete constitutive model for the material ranging across an entire shutdown or startup transient: from room temperature to operating temperature. A previous chapter evaluated two existing viscoplastic models and uncovered problems in both their representation of rate sensitivity and their treatment of work hardening. This chapter provides a method for accurately representing rate sensitivity. The next chapter describes developing and calibrating a model for work hardening and softening in Gr. 91. 



\section{Preliminary ANL constitutive model}

\subsection{Fitting a model with genetic algorithm optimization}

The previous chapter determined a model for the rate sensitivity of Gr. 91. We use the isotropic elastic constants tabulated in Section II, Part D of the ASME code for the model elastic response. A complete model must add to these a model for work hardening and softening in the material. Given the combined creep/fatigue operating conditions of advanced reactors this model must account for combined kinematic and isotropic hardening and their interaction with creep. Three families of models exist in the literature, described in more detail above: Chaboche [16, 9], Ohno and Wang [52, 53], and Krempl [43]. All three models have different formulations and their various advantages and disadvantages have been debated in the literature (c.f. [10]) but all are capable of representing the phenomena observed in Gr. 91 under creep/fatigue conditions. From a survey of commercial finite element software, the Chaboche model seems to be the most commonly implemented and so this chapter focus on this model.

A Chaboche model has three parts: classical Perzyna rate dependence, an isotropic hardening or softening model (classically Voce, but often modified by individual researchers), and a superposition of backstresses determining the kinematic hardening. Including static recovery terms often necessary to describe secondary creep each the evolution of each backstress term under isothermal conditions is

$$
\dot{\mathbf{X}}_{i}=\left(\frac{2}{3} C_{i} \mathbf{n}-\sqrt{\frac{2}{3}} \gamma_{i}\left(\bar{\varepsilon}_{p}\right) \mathbf{X}_{i}\right) \dot{\gamma}-A_{i} \sqrt{\frac{3}{2}}\left\|\mathbf{X}_{i}\right\|^{a_{i}-1}
$$

with constants $C_{i}, \gamma_{i}, A_{i}$, and $a_{i}$ to be determined from experimental data. The total backstress is the superposition of one or more individual backstress terms

$$
\mathbf{X}=\sum_{i=1}^{n} \mathbf{X}_{i}
$$

We elect to use the combined Voce hardening/linear softening isotropic model described above

$$
\sigma_{i s o}=R_{1}\left(1-e^{-\delta_{1} \bar{\varepsilon}_{p}}\right)-\delta_{2} \bar{\varepsilon}_{p}
$$

with $\bar{\varepsilon}_{p}$ the equivalent plastic strain and $R_{1}, \delta_{1}$, and $\delta_{2}$ constants to be determined.

Under non-isothermal conditions the temperature rate contributes to the evolution of these internal variables. Whether this temperature contribution can be neglected depends on both the heating/cooling rate experience over a plant loading cycle and on the values of the constants defining the model.

After selecting the number of backstresses $n$ conceptually the process of calibrating the model at a given temperature is simple: for a set of parameters $\left\{C_{i}, \gamma_{i}, A_{i}, a_{i}, R_{1}, \delta_{1}, \delta_{2}\right\}$ run, for each experiment in the database, a corresponding simulation. Form a residual comparing the experimental results to the model prediction. Form a total residual by combining the weighted residuals from each experiment into a single scalar. Now the parameter calibration process is an optimization problem: minimize this total residual.

The final model must be temperature dependent. To do this we use the concept of temperature control points. A set of parameters is defined at each temperature in a set 
of temperatures $\left\{T_{i}\right\}$. For model evaluations at temperatures between these control points parameters are linearly interpolated and the model evaluated with those interpolated parameters. Note this scheme is not the same as calibrating the model at fixed temperatures and then interpolating the parameters between those points because the optimization method used to select the parameters will carry through the interpolation. That is, the parameters at the temperature control points will be influenced by experiments at other temperatures via the optimization over the interpolated model. This should avoid the problems in the Koo and Kwon [41] model, where parameters are only provided for discrete temperature points with no explicitly provided interpolation scheme.

Several details make this a difficult optimization problem to solve. First, establishing the gradient and Hessian of this residual is extremely difficult, meaning we must resort to gradient free methods. This is not a large disadvantage, as local optimization strategies are not optimal for this parameter estimation problem: there will likely be numerous local minima we would in any event like to find a global minimum. Establishing the appropriate weights for each experimental residual requires balancing the relative importance of each type of loading with the number of available experiments and the confidence in each set of experimental data. Finally, the Gr. 91 experimental database collects over 300 tests so each evaluation of the complete residual requires 300 full viscoplastic simulations. This requires a large amount of computing time and most gradient free optimization problems require many evaluations of the residual on each iteration of the algorithm.

To decrease the time required to evaluate the residual we implemented a parallel evaluation protocol using MPI to distribute the set of experiments/simulations to a large number of processors (typically 80-100). The actual number of simulations assigned to each processor are determined by an a priori approximation of the run time required for each simulation. For example, the cyclic experiments take much longer to simulate because of the large cycle count. Currently we use a simple residual weighting scheme. Consider the five basic types of experiments available: tension, strain-controlled cyclic, stress-controlled cyclic, creep, and stress relaxation. If in each category there are $m_{i}$ available tests the current method sets the weight of each experiment in that category to $1 / m_{i}$. Essentially this weights each type of behavior equally.

We use a genetic algorithm (GA) optimization scheme to solve the gradient free global optimization problem. Genetic optimization algorithms solve a global optimization problem by an analogy to biological evolution [21,51]. The algorithms maintain a population of competing models, here each represented by a set of hardening parameters. For each iteration of the algorithm the method evaluates the weighted residual of each member of the current population, called the current or parent generation, through the parallel process described above. These residual scores are called the fitness of each member of the population. Typically, GAs are posed as maximization problems so here we define the fitness of each member of the population as the opposite of the residual. Then the GA selects the best members of the population - those with the highest fitness - through some selection scheme. Pairs of these best individuals are created and these pairs are used to produce new individuals for the next, child population through some breeding or crossover scheme. Finally, mutation operators may randomly change characteristics of the new population of children. Finally, the child generation becomes the parent generation for the next iteration of the algorithm. The process stops after some user-defined criteria is reached, for example the change in the 


\begin{tabular}{ccc}
\hline Symbol & Description & Value \\
\hline$n$ & Population size & 50 \\
$e$ & Elitism & 5 \\
$k$ & Tournament size & 3 \\
$\xi$ & Crossover probability & 0.5 \\
$\alpha$ & Individual mutation probability & 0.2 \\
$\beta$ & Per-parameter mutation probability & 0.05 \\
$\eta$ & Polynomial mutation parameter & 2.0 \\
\hline
\end{tabular}

Table 6.1: Optimization hyperparameters used in this subsection.

residual of the best member of the population over two generations is small. The final result of the scheme is the parameter set corresponding to the best member of the final generation - the optimal set of parameters.

Genetic algorithms were originally defined for parameter sets describe by binary strings. However, the methods extend to lists of real numbers, as for our particular problem. Let the population size be $n$. We elected to use a tournament selection strategy to choose pairs for crossover with tournament size $k$ and probability $p$ and elitism $e$. The best $e$ members of the parent generation are directly transferred to the child generation without crossover or mutation. Then each of the $n-e$ available slots are filled two at a time through a crossover strategy. Each member of each pair is selected through a tournament: choose $k$ members of the parent generation randomly and select the most fit. Each pair creates two children through a two point crossover strategy with probability $\xi$. With probability $\xi-1$ directly transfer the pair into the child population. With probability $\xi$ select two points at random in the two member's chromosomes - the list of parameters describing the model. Swap the parameters between these two points between the two parents to create two new children. Finally, we perform bounded polynomial mutation with probability $\alpha$, gene probability $\beta$, and crowding degree $\eta$. For each child with probability $1-\alpha$ pass the child to the next generation. With probability $\alpha$ mutate the child first. Loop over each parameter in the set. For each parameter, with probability $\beta$ perform a bounded polynomial mutation by replacing that parameter with

$$
\begin{gathered}
p_{\text {new }}= \begin{cases}p_{\text {old }}+\delta_{L}\left(p-x^{(L)}\right) & u \leq 0.5 \\
p_{\text {old }}+\delta_{R}\left(x^{(U)}-p\right) & u>0.5\end{cases} \\
\delta_{L}=\quad(2 u)^{1 /(1+\eta)}-1 \\
\delta_{R}=1-(2(1-u))^{1 /(1+\eta)}
\end{gathered}
$$

where $u$ is a random number between 0 and $1, \eta$ is a parameter, and $x^{(L)}$ and $x^{(U)}$ are lower and upper bounds on the parameter, which must also be supplied by the user [22]. Finally, the child population becomes the parent population of the next generation.

The parameters $n, e, k, \xi, \alpha, \beta$, and $\eta$ are hyperparameters which must be selected or tuned to produce good convergence. Table 6.1 lists the values used in this study, selected through a combination of a literature survey and trial and error. The genetic algorithm 


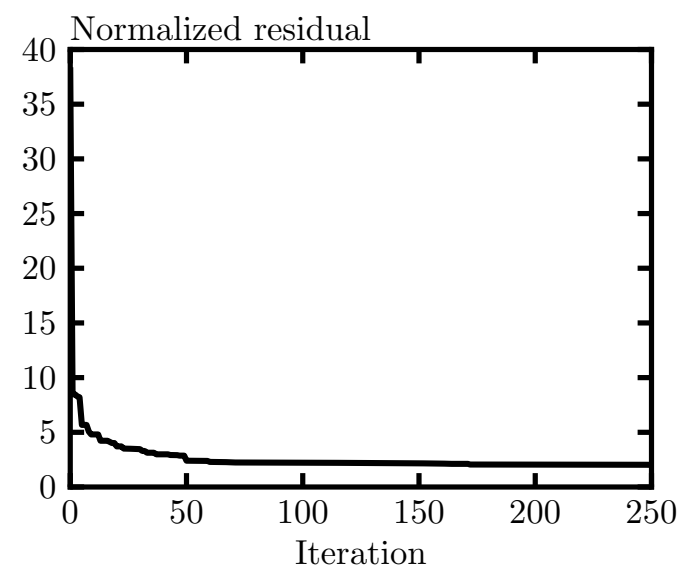

Figure 6.1: Plot illustrating the convergence of the genetic algorithm optimization method.

optimization is implemented in Python using the DEAP library [23] using PyMPI to evaluate the fitness of the population in parallel. The model of model backstresses is also a hyperparameter that cannot be selected through the optimization. In this section we use two backstresses, matching the choice of Yaguchi and Takahashi [66]. The parameter bounds are selected as to only help keep the model in a feasible region where the simulations of each experiment can converge and not to limit the optimization procedure. Figure 6.1 demonstrates that with these hyperparameters the optimization scheme convergences with a few hundred iterations to a stable, minimum residual.

Figures 6.2 - 6.7 summarize results from the "naive" GA optimization approach where the algorithm initializes with 50 random individual - 50 random parameter sets within the provided bounds. These plots compare a series of experiments and corresponding results from three models: the current, GA optimized model, the Koo \& Kwon model, and the Yaguchi \& Takahashi model. Each plot shows results for a certain type of loading, varying a single loading parameter. In order:

1. Uniaxial tension at differing strain rates.

2. Creep at differing hold stresses.

3. Stress relaxation at differing initial strains.

4. Stress controlled cycling with $\sigma_{\max }=400 \mathrm{MPa}$ and different stress ratios.

5. Stress controlled cycling with fully reversed loading and variable hold times.

6. Stress controlled cycling with fully reversed loading at different strain rates.

Where there is available data each plot has two subfigures: a) at $T=550^{\circ} \mathrm{C}$ and b) at $T=600^{\circ} \mathrm{C}$.

The automatically-calibrated model shows good results for uniaxial loading, generally outperforming the existing models. Its performance is also reasonable for creep and stress 
FY17 Status Report on the Initial Development of a Constitutive Model for Grade 91 Steel July 2017

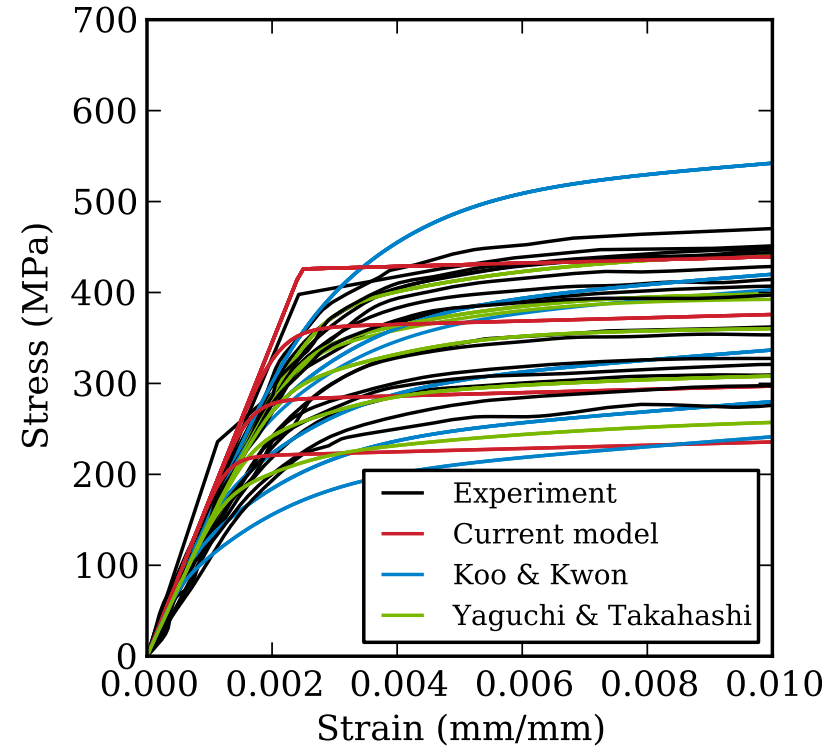

(a)

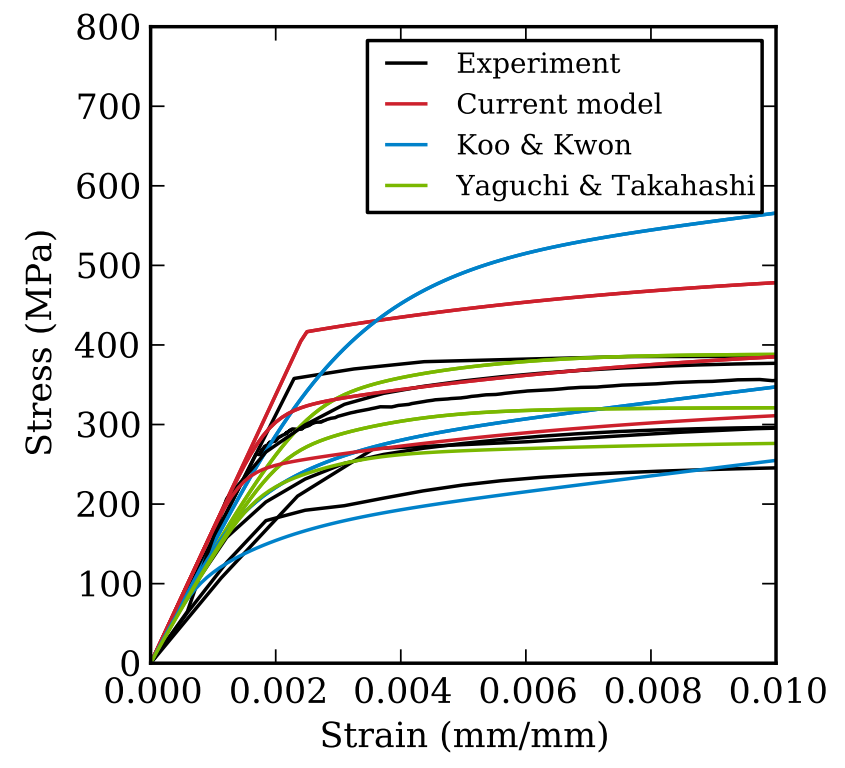

(b)

Figure 6.2: Plot comparing the GA model to experimental data, the Koo \& Kwon model, and the Yaguchi model for uniaxial tension loading at various strain rates. Subfigure a) shows results for $T=550^{\circ} \mathrm{C}$ and b) shows results for $T=600^{\circ} \mathrm{C}$.

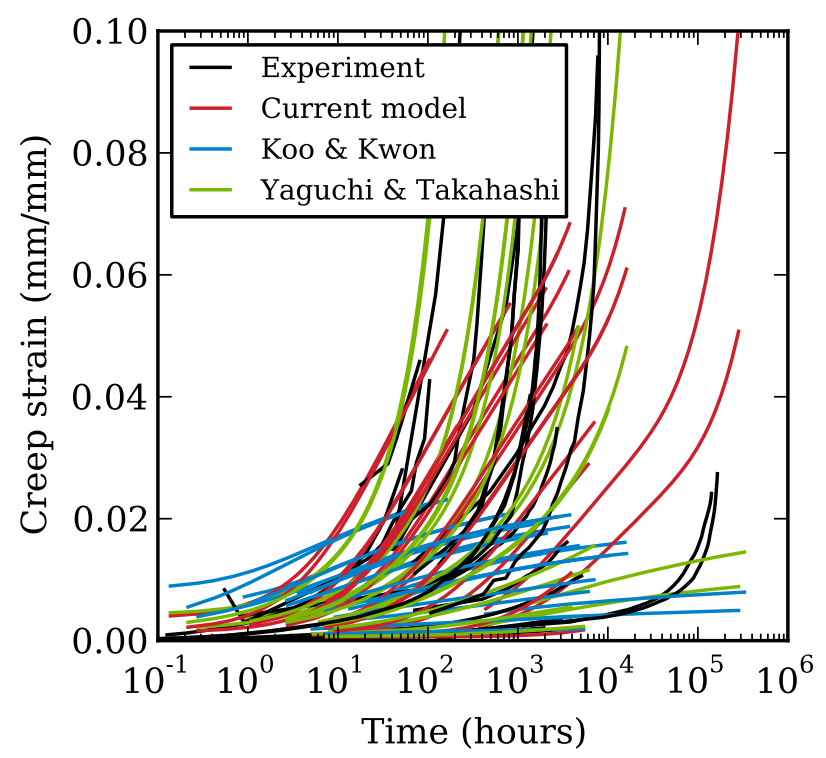

(a)

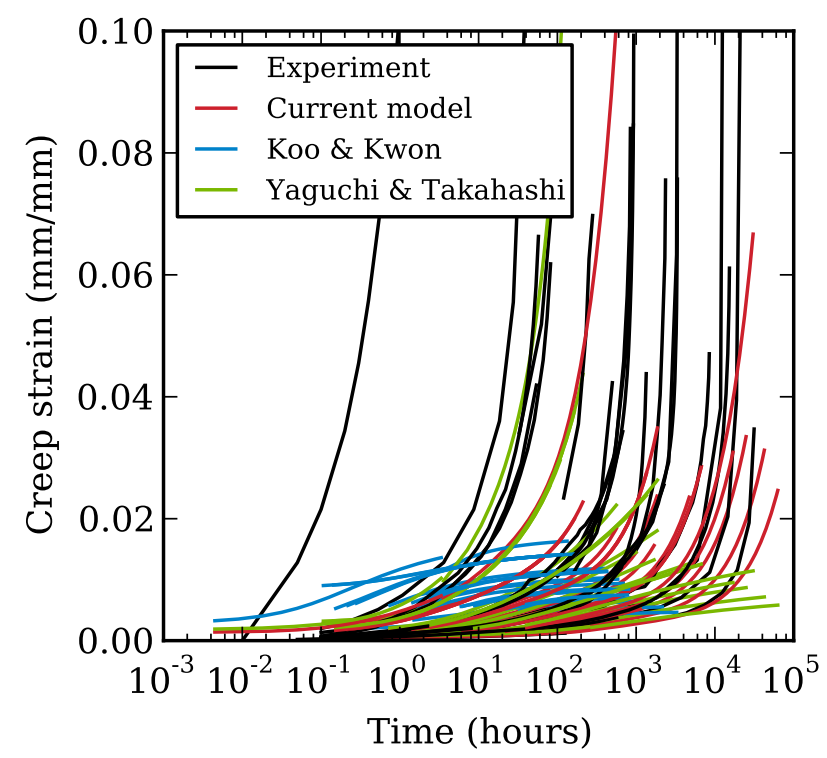

(b)

Figure 6.3: Plot comparing the GA model to experimental data, the Koo \& Kwon model, and the Yaguchi model for creep loading at various stresses. Subfigure a) shows results for $T=550^{\circ} \mathrm{C}$ and $\mathrm{b}$ ) shows results for $T=600^{\circ} \mathrm{C}$. 
FY17 Status Report on the Initial Development of a Constitutive Model for Grade 91 Steel July 2017

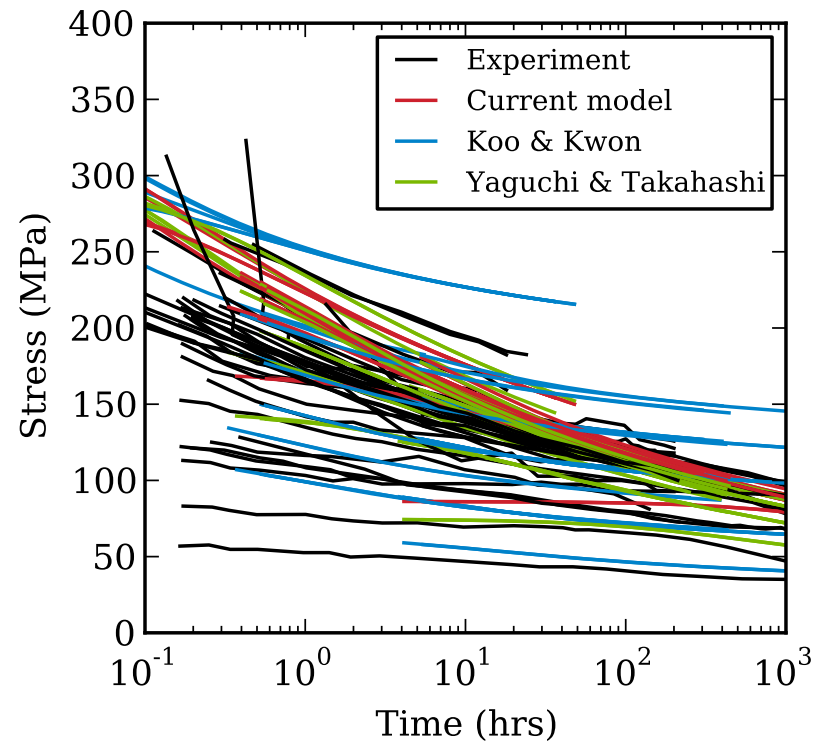

(a)

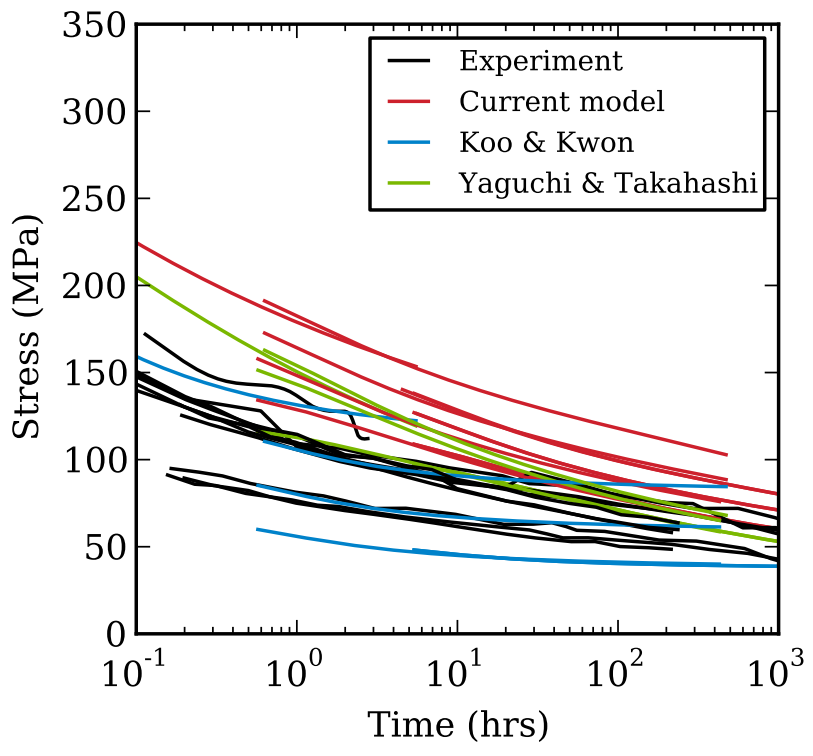

(b)

Figure 6.4: Plot comparing the GA model to experimental data, the Koo \& Kwon model, and the Yaguchi model for stress relaxation at at various initial strains. Subfigure a) shows results for $T=550^{\circ} \mathrm{C}$ and b) shows results for $T=600^{\circ} \mathrm{C}$.

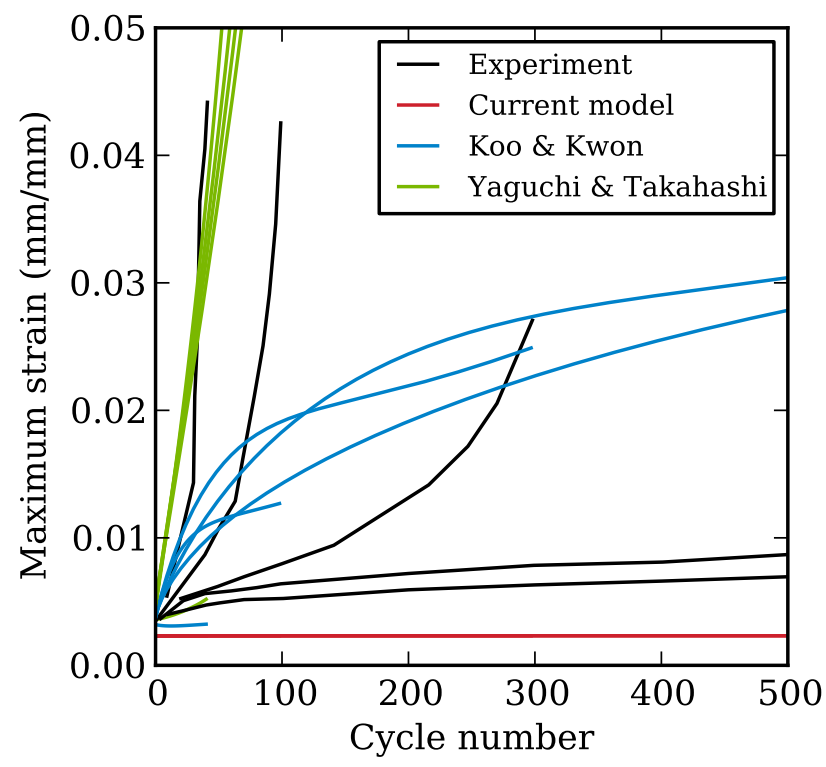

Figure 6.5: Plot comparing the GA model to experimental data, the Koo \& Kwon model, and the Yaguchi model for stress controlled cyclic loading at various stress ratios at $T=550^{\circ} \mathrm{C}$. 
FY17 Status Report on the Initial Development of a Constitutive Model for Grade 91 Steel July 2017

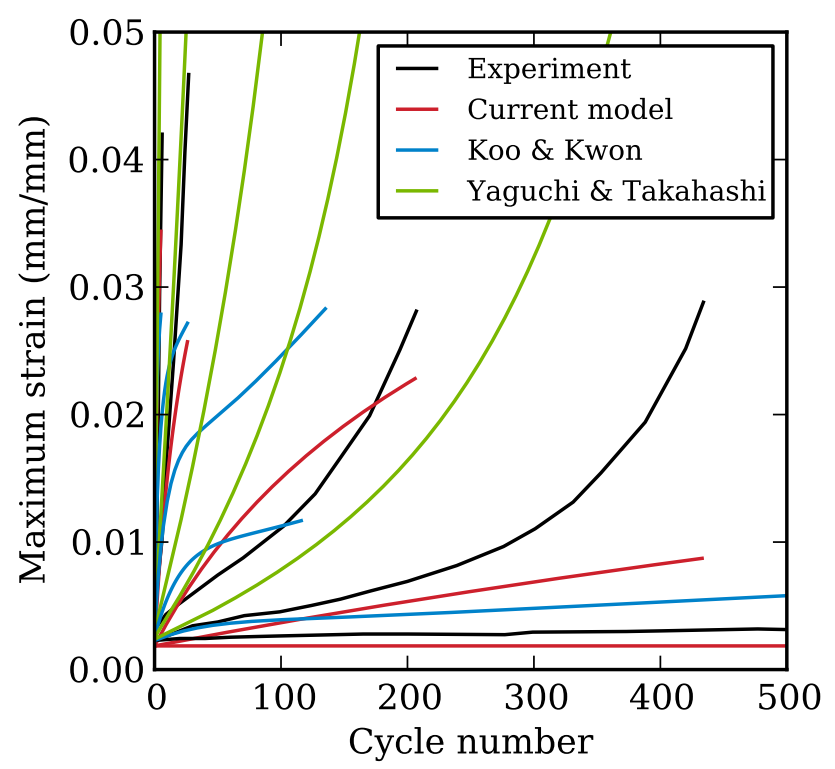

Figure 6.6: Plot comparing the GA model to experimental data, the Koo \& Kwon model, and the Yaguchi model for fully-reversed stress controlled cyclic loading with various hold times at peak load at $T=550^{\circ} \mathrm{C}$.

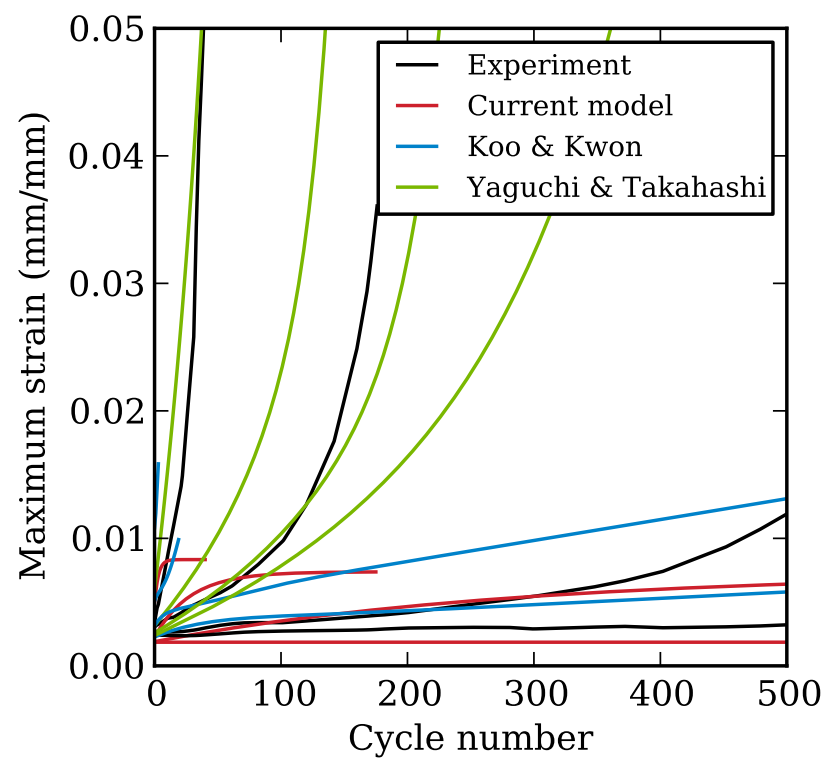

Figure 6.7: Plot comparing the GA model to experimental data, the Koo \& Kwon model, and the Yaguchi model for fully-reversed stress controlled cyclic loading with various stress rates at $T=550^{\circ} \mathrm{C}$. 
relaxation, again outperforming existing models. However, for stress-controlled cyclic deformation all three models have difficulty, particularly in capturing the effect of stress ratio. Gr. 91 ratchets even under fully-reversed cyclic loading, likely due to tension/compression asymmetry $[67,68]$. None of these models attempt to capture this asymmetry and so do not correctly represent material ratcheting effects. Furthermore the model has some difficulty capturing ratcheting softening - increasing ratcheting rates with increasing cycles, a behavior that that Yaguchi model correctly captures.

However, the automatically-calibrated model is approximately as accurate as these figures indicate across the entire temperature range - room temperature to $650^{\circ} \mathrm{C}$. Koo \& Kwon only attempted to fit coefficients for $T=500^{\circ} \mathrm{C}, T=550^{\circ} \mathrm{C}$, and $T=600^{\circ} \mathrm{C}$ and Yaguchi \& Takahashi only provide interpolation formula in between $200^{\circ} \mathrm{C}$ and $600^{\circ} \mathrm{C}$. Furthermore, because the calibrating process is automatic it is straightforward to fit a new model with a new form or with a modified optimization method.

\subsection{Improved calibration strategy}

Several defects of the GA optimization strategy were described in the previous section. The optimization results are sensitive to the initial population and the selected parameter ranges. The model can sacrifice a solution that, with the benefit of engineering judgment, seems better overall to reduce the residual on another type of test or at another temperature by sacrificing one particular experiment/simulation pair. One way to overcome these problems is to "seed" the initial population with manually-calibrated parameter sets. This has the advantage of introducing engineering judgement into the process without sacrificing the formal minimization of the residual over the complete data set.

Manually calibrating a parameter set can be a very long and tedious even at a single temperature. Figures 6.8 to 6.13 present results for a manually tuned model at $T=550^{\circ} \mathrm{C}$. These plots are of the same type as Figs. 6.2 - 6.7 above.

This manually-tuned model outperforms the existing models for all types of loading, with the exception of material ratcheting under different stress ratios. Again, all three models fail to capture the observed material response because none of the models incorporates tension/compression asymmetry. However, the results of the manual calibration process provide a clear path forward for improving the overall model results: manually calibrate a few other temperature control points, add this set of parameters to the initial GA population, and have the GA optimization automatically improve the model to optimize its performance.

\subsection{Conclusions and ongoing work}

This section demonstrates a complete framework for calibrating inelastic material models. With sufficient data, already collected for Gr. 91, GA algorithms started with manuallycalibrated exemplar individuals can automatically tune and improve the model parameters to match the average material response. This is a critical point: all work on Gr. 91, and most work on other structural materials, typically aims to calibrate a model to a particular set of data, typically a series of experiments performed by the researcher. This approach is not adequate for inclusion in the ASME code. Such a model must capture the average 
FY17 Status Report on the Initial Development of a Constitutive Model for Grade 91 Steel July 2017

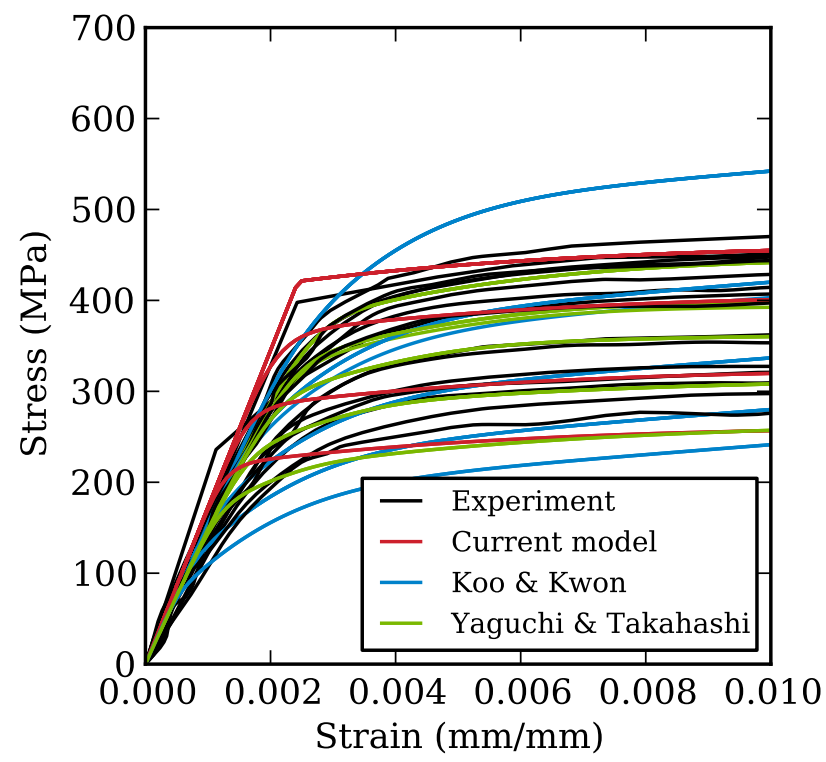

Figure 6.8: Plot comparing the manually calibrated model to experimental data, the Koo \& Kwon model, and the Yaguchi model for uniaxial tension loading at various strain rates at $T=550^{\circ} \mathrm{C}$.

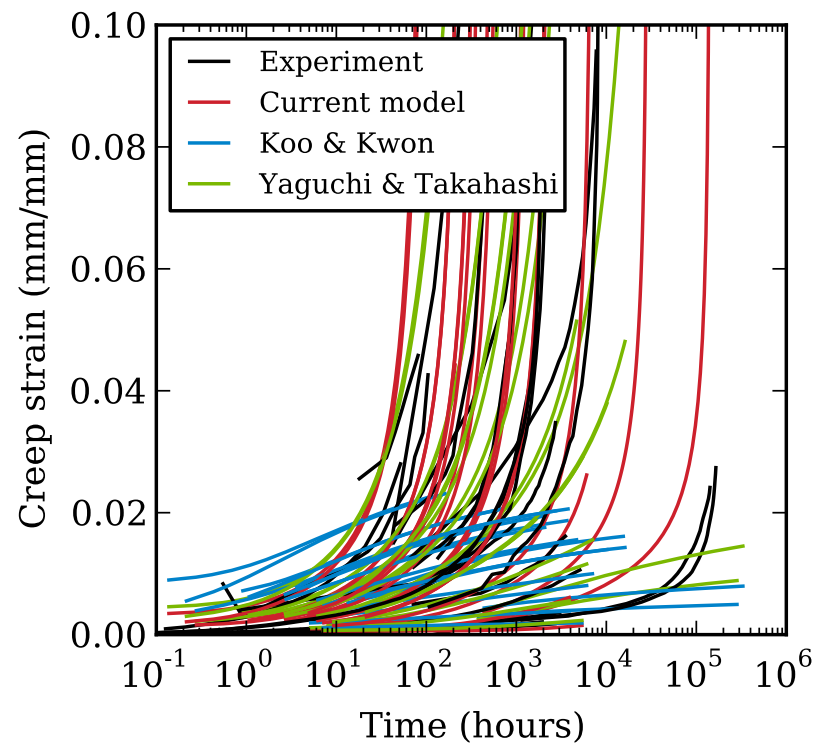

Figure 6.9: Plot comparing the manually calibrated model to experimental data, the Koo \& Kwon model, and the Yaguchi model for creep loading at various stresses at $T=550^{\circ} \mathrm{C}$. 
FY17 Status Report on the Initial Development of a Constitutive Model for Grade 91 Steel July 2017

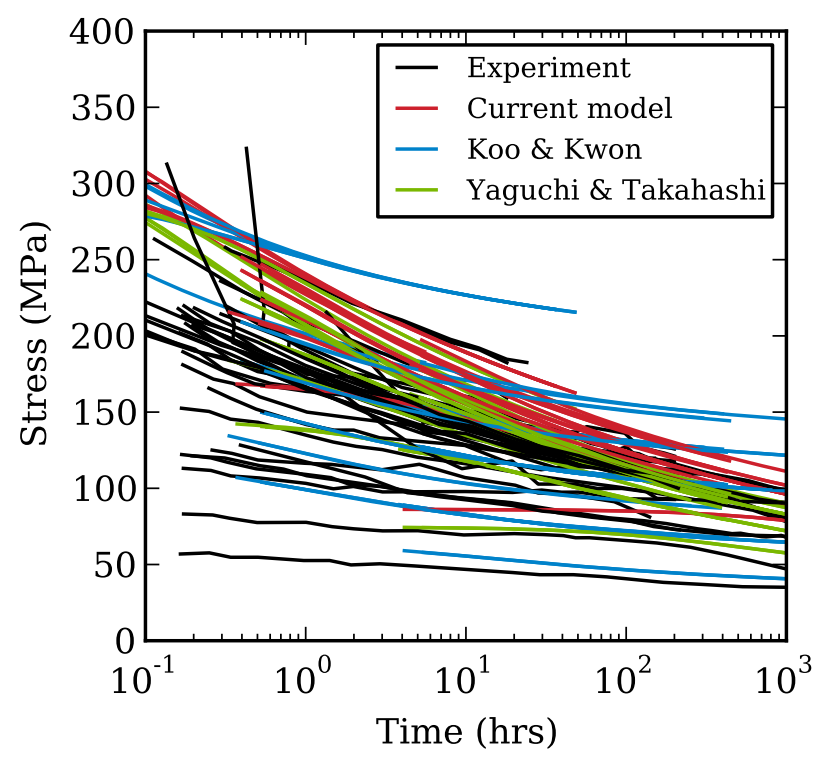

Figure 6.10: Plot comparing the manually calibrated model to experimental data, the Koo \& Kwon model, and the Yaguchi model for stress relaxation at at various initial strains at $T=550^{\circ} \mathrm{C}$.

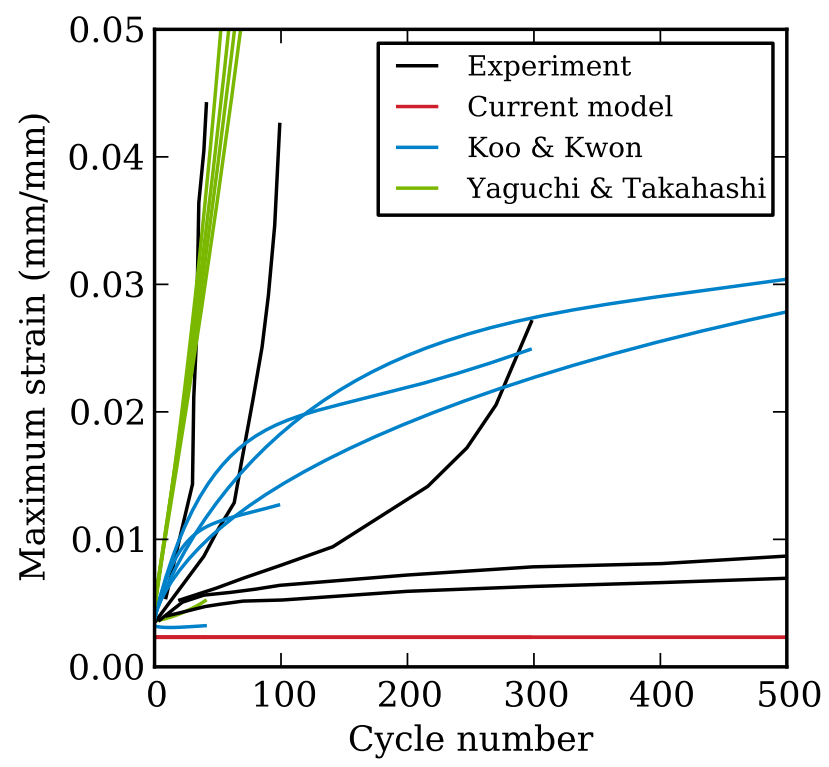

Figure 6.11: Plot comparing the manually calibrated model to experimental data, the Koo \& Kwon model, and the Yaguchi model for stress controlled cyclic loading at various stress ratios at $T=550^{\circ} \mathrm{C}$. 
FY17 Status Report on the Initial Development of a Constitutive Model for Grade 91 Steel July 2017

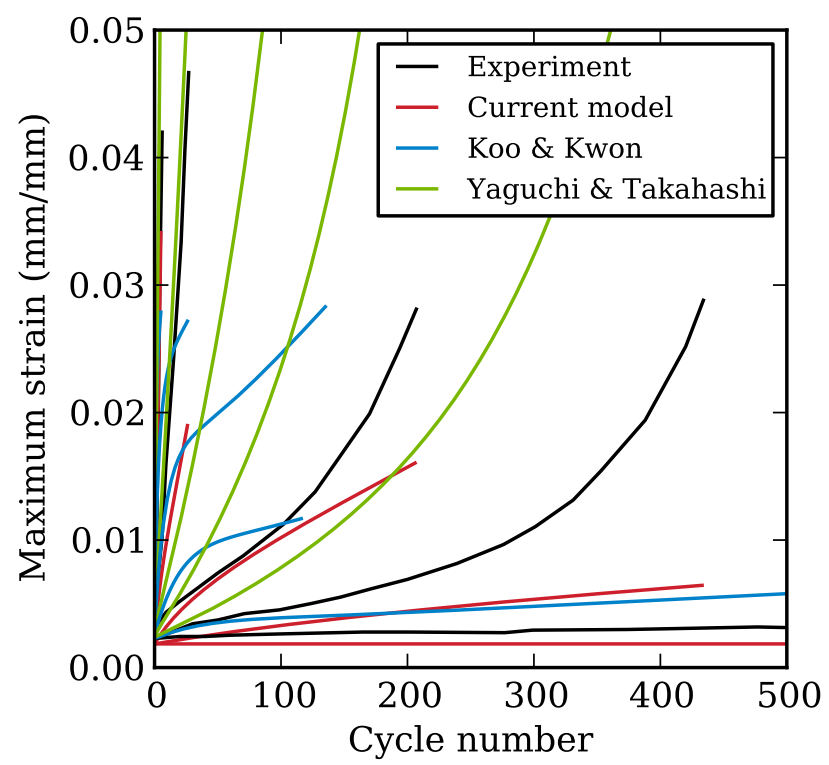

Figure 6.12: Plot comparing the manually calibrated model to experimental data, the Koo \& Kwon model, and the Yaguchi model for fully-reversed stress controlled cyclic loading with various hold times at peak load at $T=550^{\circ} \mathrm{C}$.

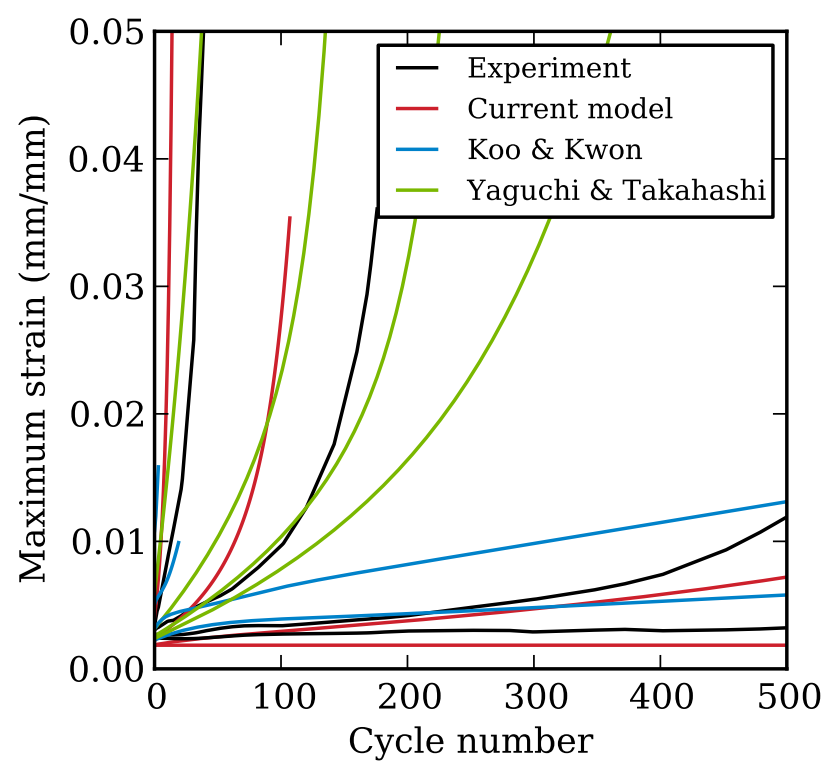

Figure 6.13: Plot comparing the manually calibrated model to experimental data, the Koo \& Kwon model, and the Yaguchi model for fully-reversed stress controlled cyclic loading with various stress rates at $T=550^{\circ} \mathrm{C}$. 
behavior of the material, including all material batch variation. Furthermore, the final model must be well supported by a large quantity of experimental data in order to give designers confidence in using the model for advanced reactor design calculations.

While the Gr. 91 data set is extensive it still has gaps that makes calibrating an accurate model across the full temperature range difficult. For example, there is limited low temperature $\left(<200^{\circ} \mathrm{C}\right)$ data available in the literature and more experiments are required to fully characterize material ratcheting across the full operating temperature range. These needs require close collaboration with the ongoing experimental program on Gr. 91.

One gap in all the models considered here is the lack any mechanism for developing tension/compression asymmetry, which impacts the performance of the models when compared to experimental ratcheting data. Future work will investigate approaches for accounting for these effects. 


\section{Conclusions and future work}

\subsection{Experimental data and existing models}

This report describes a substantial collection of experimental data on Gr. 91 - over 300 experiments all standardized into a machine-readable XML format for easy access. Five previous Gr. 91 material models were identified and two implemented and compared to experimental data.

Future experimental work should focus on filling in gaps in the experimental database in order to cover the full temperature range, from room temperature to the maximum use temperature of $650^{\circ} \mathrm{C}$ required in the Code for Gr. 91. Additional experiments are required to better-characterize material ratcheting at all temperatures. Furthermore, the experimental database should be updated as new information becomes available, for example long term creep experiments currently in progress.

Finally, looking ahead, integrated experiments on realistic plant geometries are needed for the final validation of material models for Gr. 91 and other advanced reactor structural materials. Some such experiments were performed in the past supported by DOE and industry, but new experimental techniques such as high resolution digital image correlation (DIC) could provide better experimental data that can better validate simulation results and provide greater confidence in inelastic modeling to the design community. These experiments could provide the "ground truth" need to promote wider adoption of design by inelastic analysis, potentially leading to more efficient, economical plant designs.

\subsection{New constitutive models}

An optimization framework was develop to calibrate a particular model form to match the average response of a large series of experimental tests. This approach is in contrast to previous model development efforts which tend to look at a single set of experimental data. The approach of matching the average response of a large experimental database is more appropriate for a model to be included in the ASME code.

One rational approach for fitting a model to a large data set with high scatter caused by batch variation is to pose a formal optimization problem. This report describes formulating such an optimization problem and then searching for a global minimum via genetic algorithm optimization and performs the optimization over the current experimental database to generate a preliminary, trial model. The results of this naive GA optimization strategy can be improved by including manually-calibrated "exemplar" models in the initial GA population. This report calibrated such an exemplar for $550^{\circ} \mathrm{C}$.

Calibrating a final Gr. 91 model has three prerequisites: 1) collecting experimental data to fill in the lower temperature gaps in the experimental database, 2) fitting more exemplars at other temperatures, and 3) including a mechanism in the model to allow for tension/compression asymmetry, to capture material ratcheting under fully-reversed loading. These tasks will be addressed in next fiscal year to produce a final constitutive model. 



\section{A XML Data Format}

The experimental data collected for this report have been collated into an XML format for easy querying and sampling. A separate XML file with root xml tag <material name="'gr91" > collects the data from a group of experiments. This XML database format could be extended in the future to cover other materials of interest by adding entries with different material names. A simple python script merges all the XML files into a single XML database under a common root tag, merging all data for a given material into the same sub tag.

Each material tag contains a collection of experiments, described by the <experiment> tag. The type attribute of this tag describes the type of experiment: monotonic, cyclic, or relaxation with each type allowing for either stress or strain controlled deformation. The example at the end of this appendix shows one instance of each experiment type, demonstrating the data and metadata stored for each experiment.

Storing the experimental data in an XML database allows a user to easily query the database for particular experiments of interest using the XPath syntax. For example, the query

experiment [Qtype="cyclic"] [control="stress"] [value>390.0] [ratio<-0.5]

finds all stress controlled cyclic experiments with applied stress greater than $390 \mathrm{MPa}$ and stress ratio less than -0.5 . As another example the query

experiment [@type="monotonic"] [control="strain"] [temperature $>525]$

[temperature<575] [strain]

finds all strain controlled monotonic experiments (i.e. monotonic tension tests) conducted at temperatures between $525 \mathrm{~K}$ and $575 \mathrm{~K}$ and containing full stress/strain data, as some experiments only report yield and ultimate tensile stress.

The implementation of this database includes python scripts to load text data from the XML files and convert it to a numerical format - either python floating point numbers or numpy arrays. Additionally, the scripts automatically handle the conversion of units between different systems. These units are included in the xml data using units attributes on tags holding the data. Additional scripts can post-process data to include useful additional information in the XML files. For example, scripts can generate yield stresses from stress/strain data and creep rates from creep strain versus time information.

The following provides an example XML file for a set of experiments, showing one entry for each type of experiment that the report identified for Gr. 91. In this listing ellipses (...) indicate information omitted from the reproduction here but that would be present in the actual file. For example, only the first few entries in a stress/strain curve are reproduced to avoid overflowing the page.

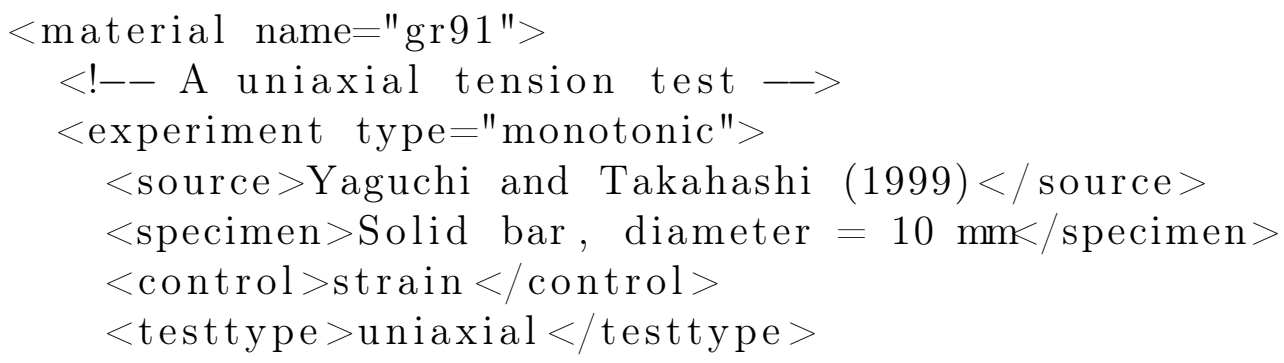


$<$ direction $>$ unknown $</$ direction $>$

$<$ tc $>$ tension $</$ tc $>$

$<$ pressure units $=" \mathrm{MPa} ">0.0</$ pressure $>$

$<$ temperature units $=$ "kelvin " $>823.15</$ temperature $>$

$<$ rate units $=" 1 /$ second $">0.001</$ rate $>$

$<$ strain units $="$ dimensionless " $>0.0,0.0002092, \ldots</$ strain $>$

$<$ stress units $=" \mathrm{MPa} ">0.0,41.7, \ldots</$ stress $>$

$<$ time units $=$ "second" $>0.0,0.2092, \ldots</$ time $>$

$<$ youngs units $=" M P a ">138075.630898</$ youngs $>$

$<$ yield units $=" M P a ">420.339481696</$ yield $>$

$</$ experiment $>$

$<$ !-- A stress controlled cyclic test $\longrightarrow$

$<$ experiment type="cyclic" $>$

$<$ source $>$ Yaguchi and Takahashi $(2005)</$ source $>$

$<$ specimen $>$ Solid bar, diameter $=10 \mathrm{~mm}</$ specimen $>$

$<$ control $>$ stress $</$ control $>$

$<$ testtype $>$ uniaxial $</$ testtype $>$

$<$ direction $>$ unknown $</$ direction $>$

$<$ temperature units $=$ "kelvin" $>823.15</$ temperature $>$

$<$ rate units $=" \mathrm{MPa} / \mathrm{s}^{\prime \prime}>50.0</$ rate $>$

$<$ pressure units $=" \mathrm{MPa} ">0.0</$ pressure $>$

$<$ cycle $>9.0,18.0, \ldots</$ cycle $>$

$<$ value units $=$ "MPa" $>400.0</$ value $>$

$<$ ratio units $=$ "dimensionless" $>0.0</$ ratio $>$

$<\max$ units $=$ "dimensionless " $>0.0054348,0.0092924, \ldots</ \max >$

$</$ experiment $>$

$<$ !-- A strain controlled cyclic test $\longrightarrow$

$<$ experiment type="cyclic" $>$

$<$ source $>$ Yaguchi and Takahashi $(2005)</$ source $>$

$<$ specimen $>$ Unknown $</$ specimen $>$

$<$ control $>$ strain $</$ control $>$

$<$ testtype $>$ uniaxial $</$ testtype $>$

$<$ direction $>$ unknown $</$ direction $>$

$<$ temperature units $=$ "kelvin " $>823.15</$ temperature $>$

$<$ rate units $=" 1 /$ second $">0.001</$ rate $>$

$<$ pressure units $=" \mathrm{MPa} ">0.0</$ pressure $>$

$<$ value units $=$ "dimensionless" $>0.01</$ value $>$

$<$ ratio units $=$ "dimensionless" $>-1.0</$ ratio $>$

$<$ cycle $>1.0,2.0, \ldots</$ cycle $>$

$<\max$ units $=" \mathrm{MPa} ">429.16,422.976, \ldots</ \max >$

$<\min$ units $=" M P a ">-439.824,-432.472, \ldots</ \min >$

$</$ experiment $>$ 


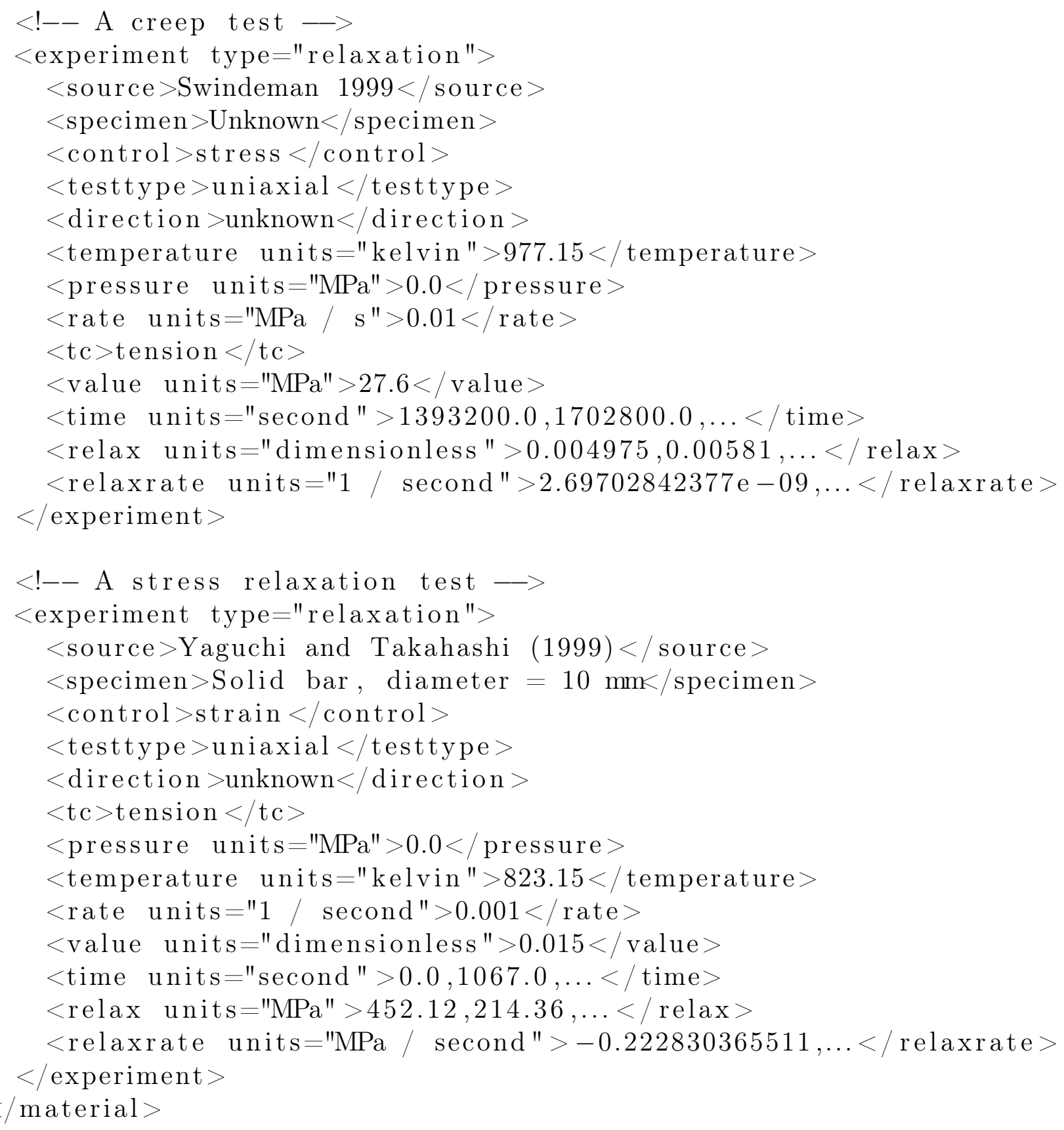





\section{Acknowledgments}

The research was sponsored by the U.S. Department of Energy (DOE), under Contract No. DE-AC02-06CH11357 with Argonne National Laboratory (ANL), managed and operated by UChicago Argonne LLC. Programmatic direction was provided by the Office of Advanced Reactor Technologies (ART) of the Office of Nuclear Energy (NE). The authors gratefully acknowledge the support provided by William Corwin of DOE-NE, ART Materials Technology Lead, and Robert Hill of ANL, ART Co-National Technical Director.

The authors acknowledge the contributions of Yanli Wang at Oak Ridge National Laboratory (ORNL) and Meimei Li at ANL. We also acknowledge Robert Jetter of R.I. Jetter Consulting for discussions on the American Society of Mechanical Engineers (ASME) Code requirements. 



\section{Bibliography}

[1] M. Abdel-Karim and N. Ohno. Kinematic hardening model suitable for ratchetting with steady-state. International journal of plasticity, 16(3):225-240, 2000.

[2] Fujio Abe. Precipitate design for creep strengthening of $9 \%$ Cr tempered martensitic steel for ultra- supercritical power plants Precipitate design for creep strengthening of 9\% Cr tempered martensitic steel for ultra-supercritical power plants. Sci. Technol. Adv. Mater. Sci. Technol. Adv. Mater, 9(9):13002-15, 2008.

[3] Andrey Belyakov, Y. Kimura, and K. Tsuzaki. Recovery and recrystallization in ferritic stainless steel after large strain deformation. Materials Science and Engineering A, 403 (1-2):249-259, 2005.

[4] O .T. Bruhns and U. Rott. A viscoplastic model with smooth transition to describe rate-independent plasticity. International Journal of Plasticity, 10(4):347-362, 1994.

[5] E Cerri, E Evangelista, S Spigarelli, and P Bianchi. Evolution of microstructure in a modified 9Cr-1Mo steel during short term creep. Materials Science and Engineering: A, 245(2):285-292, 1998.

[6] J. L. Chaboche. Anisotropic creep damage in the framework of continuum damage mechanics. Nuclear Engineering and Design, 79(3):309-319, 1984.

[7] J. L. Chaboche. Time-independent constitutive theories for cyclic plasticity. International Journal of Plasticity, 2(2):149-188, 1986.

[8] J. L. Chaboche. Continuum damage mechanics: Present state and future trends. Nuclear Engineering and Design, 105(1):19-33, 1987.

[9] J. L. Chaboche. On some modifications of kinematic hardening to improve the description of ratchetting effects. International Journal of Plasticity, 7(7):661-678, 1991.

[10] J. L. Chaboche. A review of some plasticity and viscoplasticity constitutive theories. International Journal of Plasticity, 24(10):1642-1693, oct 2008.

[11] J. L. Chaboche and G. Cailletaud. On the calculation of structures in cyclic plasticity or viscoplasticity. Computers and Structures, 23(1):23-31, 1986.

[12] J. L. Chaboche and G Cailletaudb. Integration methods for complex plastic constitutive equations. Computational Methods in Applied Mechanics and Engineering, 133(95):125$155,1996$.

[13] J. L. Chaboche and O. Jung. Application of a kinematic hardening viscoplasticity model with thresholds to the residual stress relaxation. International Journal of Plasticity, 13 (10):785-807, 1998.

[14] Jean Louis Chaboche. Continuous damage mechanics - A tool to describe phenomena before crack initiation. Nuclear Engineering and Design, 64(2):233-247, 1981. 
[15] Jean-Louis Chaboche. Thermodynamic formulation of constitutive equations and application to the viscoplasticity and viscoelasticity of metals and polymers. International Journal of Solids and Structures, 34(18):2239-2254, 1997.

[16] J.L. Chaboche. Constitutive equations for cyclic plasticty and cyclic viscoplasticity. International Journal of Plasticity, 5:247-302, 1989.

[17] P. Chellapandi, R. Ramesh, S. C. Chetal, and S. B. Bhoje. Application of Chaboche viscoplastic thoery for predictiong the cyclic behaviour of modified 9Cr-1Mo. In 14th International Conference on Structural Mechanics in Reactor Technology, pages 67-76, 1997.

[18] B. K. Choudhary and E. Isaac Samuel. Creep behaviour of modified 9Cr-1Mo ferritic steel. Journal of Nuclear Materials, 412(1):82-89, 2011.

[19] J. Christopher, B. K. Choudhary, E. Isaac Samuel, V. S. Srinivasan, and M. D. Mathew. Tensile flow and work hardening behaviour of $9 \mathrm{Cr}-1 \mathrm{Mo}$ ferritic steel in the frame work of Voce relationship. Materials Science and Engineering A, 528(21):6589-6595, 2011.

[20] R. L. Coble. A model for boundary diffusion controlled creep in polycrystalline materials. Journal of Applied Physics, 34, 1963.

[21] Lawrence Davis. Handbook of Genetic Algorithms. 1991.

[22] Kalyanmoy Deb and ayan Deb. Analysing mutation schemes for real-parameter genetic algorithms. International Journal of Artificial Intelligence and Soft Computing, 4(1):1, 2014.

[23] F-A. Fortin, F-M. De Rainville, and M-A. Gardner. DEAP: Evolutionary algorithms made easy. Journal of Machine Learning Research, 13:2171-2175, 2012.

[24] B. Fournier, M. Sauzay, C. Caës, M. Noblecourt, M. Mottot, A. Bougault, V. Rabeau, J. Man, O. Gillia, P. Lemoine, and A. Pineau. Creep-fatigue-oxidation interactions in a 9Cr-1Mo martensitic steel. Part II: Effect of compressive holding period on fagitue life. International Journal of Fatigue, 30:663-676, 2008.

[25] B. Fournier, M. Sauzay, C. Caës, M. Noblecourt, M. Mottot, A. Bougault, V. Rabeau, J. Man, O. Gillia, P. Lemoine, and A. Pineau. Creep-fatigue-oxidation interactions in a 9Cr-1Mo martensitic steel. Part III: Lifetime prediction. International Journal of Fatigue, 30(10-11):1797-1812, 2008.

[26] B. Fournier, M. Sauzay, C. Caës, M. Noblecourt, M. Mottot, A. Bougault, V. Rabeau, and A. Pineau. Creep-fatigue-oxidation interactions in a 9Cr-1Mo martensitic steel. Part I: Effect of tensile holding period on fatigue lifetime. International Journal of Fatigue, 30(4):649-662, 2008.

[27] B. Fournier, M. Salvi, F. Dalle, Y. De Carlan, C. Caës, M. Sauzay, and A. Pineau. Lifetime prediction of $9-12 \% \mathrm{Cr}$ martensitic steels subjected to creep-fatigue at high temperature. International Journal of Fatigue, 32(6):971-978, 2010. 
[28] Benjamin Fournier, M. Sauzay, F. Barcelo, E. Rauch, A. Renault, T. Cozzika, L. Dupuy, and A. Pineau. Creep-fatigue interactions in a 9 Pct Cr-1 Pct Mo martensitic steel: Part II. Microstructural evolutions. Metallurgical and Materials Transactions A: Physical Metallurgy and Materials Science, 40(2):330-341, 2009.

[29] A. D. Freed and K. P. Walker. Viscoplasticity with creep and plasticity bounds. International Journal of Plasticity, 9:213-242, 1993.

[30] B. G. Gieseke, C. R. Brinkman, and P. J. Maziasz. The influence of thermal aging on the microstructure and fatigue properties of modified $9 \mathrm{Cr}-1 \mathrm{M}$ o steel. In TMS The Minerals, Metals \&f Materials Society, 1993.

[31] J. Hald. Microstructure and long-term creep properties of 9-12\% Cr steels. International Journal of Pressure Vessels and Piping, 85(1-2):30-37, 2008.

[32] J. Harper and J. E. Dorn. Viscous creep of aluminum near its melting temperature. Acta Metallurgica, 5(November):654-665, 1957.

[33] Conyers Herring. Diffusional viscosity of a polycrystalline solid. Journal of Applied Physics, 21(5):437-445, 1950.

[34] J. P. Hirth and J. Lothe. Theory of dislocations. 1982.

[35] S Kim and J R Weertman. Investigation of Microstructural Changes in a Ferritic Steel Caused by High Temperature Fatigue. Metallurgical Transactions, 19A(April):999-1007, 1988.

[36] K. Kimura, H. Kushima, and K. Sawada. Long-term creep deformation property of modified 9Cr-1Mo steel. Materials Science and Engineering A, 510-511(C):58-63, 2009.

[37] Luboš Kloc and Václav Sklenička. Transition from power-law to viscous creep behaviour of P-91 type heat-resistant steel. Materials Science and Engineering A, 234-236:962-965, 1997.

[38] U .F. Kocks. Kinematics and Kinetics of Plasticity. In U. F. Kocks, C. N. Tome, and H-R Wenk, editors, Texture and Anisotropy, chapter 8, pages 327-389. 1998.

[39] U F Kocks. Realistic constitutive relation for metal plasticity. Materials Science and Engineering A, 317:181-187, 2001.

[40] U. F. Kocks, A. S. Argon, and M. F. Ashby. Thermodynamics and kinetics of slip. In B. Chalmers, J. W. Christian, and T. B. Massalski, editors, Progress in Materials Science, pages 64-291. 1975.

[41] Gyeong H. Koo and Ji Hyun Kwon. Identification of inelastic material parameters for modified 9Cr-1Mo steel applicable to the plastic and viscoplastic constitutive equations. International Journal of Pressure Vessels and Piping, 88(1):26-33, 2011. 
[42] Gyeong H. Koo and Jae H. Lee. Investigation of ratcheting characteristics of modified $9 \mathrm{Cr}$-1Mo steel by using the Chaboche constitutive model. International Journal of Pressure Vessels and Piping, 84(5):284-292, 2007.

[43] E. Krempl. Models of viscoplasticity some comments on equilibrium (back) stress and drag stress. Acta Mechanica, 69(1-4):25-42, 1987.

[44] E. Krempl. Creep-Plasticity Interaction. In Creep and Damage in Materials and Structures, pages 285-348. 1999.

[45] S. Latha, M. Nandagopal, S. Panneer Selvi, K. Laha, and M. D. Mathew. Tensile and creep behaviour of modified $9 \mathrm{Cr}$-1Mo steel cladding tube for fast reactor using metallic fuel. Procedia Engineering, 86:71-79, 2014.

[46] J. Lemaitre and J. L. Chaboche. Mechanics of Solid Materials. Cambridge University Press, 1994.

[47] Kouichi Maruyama, Junya Nakamura, Kyosuke Yoshimi, and Yuji Nagae. Evaluation of long-term creep rupture life of Gr.91 steel by analysis of on-going creep curves. In Proceedings from the Eighth International Conference on Advances in Materials Technoglogy for Fossil Power Plants, pages 467-478, 2016.

[48] H. Mecking and U. F. Kocks. Kinetics of flow and strain-hardening. Acta Metallurgica, 29:1865-1875, 1981.

[49] H Mecking, B Nicklas, N. Zarubova, and U F Kocks. A "universal" temperature scale for plastic flow. Acta Metallurgica, 34(3):527-535, 1986.

[50] J C Moosbrugger. Nonisothermal constitutive model for the small strain behavior of 9 Cr-1 Mo-V-Nb pressure vessel steel. Journal of Engineering Materials and Technology, 114(4):354-361, 1992.

[51] H. Muehlenbein and D. Schlierkamp-Voosen. Predictive models for the breeder genetic algorithm I. Continous parameter optimization. Evolutionary Computation, 1(1):25-49, 1993.

[52] N. Ohno and J.-D. Wang. Kinematic hardening rules with critical state of dynamic recovery, part I: formulation and basic features for ratchetting behavior. International Journal of Plasticity, 9(3):375-390, 1993.

[53] N. Ohno and J.-D. Wang. Kinematic hardening rules with critical state of dynamic recovery, part II: Application to experiments of ratchetting behavior. International Journal of Plasticity, 9(3):391-403, 1993.

[54] Djordje Peric. On a class of constitutive equations in viscoplasticity: Formulation and computational issues. International Journal for Numerical Methods in Engineering, 36 (8):1365-1393, 1993. 
[55] C. Roucoules, M. Pietrzyk, and P. D. Hodgson. Analysis of work hardening and recrystallization during the hot working of steel using a statistically based internal variable model. Materials Science and Engineering A, 339(1-2):1-9, 2003.

[56] A. Seeger, J. Diehl, S. Mader, and H. Rebstock. Work-hardening and work-softening of face-centred cubic metal crystals. Philosophical Magazine, 2(15):323-350, 1957.

[57] T.-L. Sham and Kevin P. Walker. Preliminary development of a unified viscoplastic constitutive model for Alloy 617 with special reference to long term creep behavior. In Fourth International Topical Meeting on High Temperature Reactor Technology, pages 81-89, 2008.

[58] Triratna Shrestha, Mehdi Basirat, Indrajit Charit, Gabriel P. Potirniche, Karl K. Rink, and Uttara Sahaym. Creep deformation mechanisms in modified 9Cr-1Mo steel. Journal of Nuclear Materials, 423(1-3):110-119, 2012.

[59] Triratna Shrestha, Mehdi Basirat, Indrajit Charit, Gabriel P. Potirniche, and Karl K. Rink. Creep rupture behavior of Grade 91 steel. Materials Science and Engineering A, 565:382-391, 2013.

[60] J. C. Simo and T. J. R. Hughes. Computational Inelasticity. Springer-Verlag, New York, 1998.

[61] R. W. Swindeman. Cyclic Stress-Strain-Time Response of a 9CMMo-V-Nb Pressure Vessel Steel at High Temperature. In H. D. Solomon, G. R. Halford, L. R. Kaisand, and B. N. Leis, editors, Low Cycle Fatigue, ASTM STP 942, pages 107-122. American Society for Testing and Materials, Philadelphia, 1988.

[62] R. W. Swindeman. Construction of isochronous stress-strain curves for 9Cr-1Mo-V Steel. Advances in Life Prediction Methodology, 391:95-100, 1999.

[63] K. C. Valanis. A theory of visco-plasticity with out a yield surface, Part I: general theory. Archives of Mechanics, 23:517-551, 1971.

[64] K. P. Walker and E. Krempl. An implicit functional theory of viscoplasticity. Mechanics Research Communications, 5(4):179-184, 1978.

[65] M. Yaguchi and Y. Takahashi. Unified Inelastic Constitutive Model for Modified 9Cr1Mo Steel Incorporating Dynamic Strain Aging Effect. JSME International Journal, Series A, 42(1):1-10, 1999.

[66] Masatsugu Yaguchi and Yukio Takahashi. A viscoplastic constitutive model incorporating dynamic strain aging effect during cyclic deformation conditions. International Journal of Plasticity, 16(3-4):241-262, 2000.

[67] Masatsugu Yaguchi and Yukio Takahashi. Ratchetting of viscoplastic material with cyclic softening, part 1: Experiments on modified 9Cr-1Mo steel. International Journal of Plasticity, 21(1):43-65, 2005. 
FY17 Status Report on the Initial Development of a Constitutive Model for Grade 91 Steel July 2017

[68] Masatsugu Yaguchi and Yukio Takahashi. Ratchetting of viscoplastic material with cyclic softening, part 2: application of constitutive models. International Journal of Plasticity, 21:835-860, 2005. 
FY17 Status Report on the Initial Development of a Constitutive Model for Grade 91 Steel July 2017

\section{Distribution list}

$\begin{array}{lll}\text { Name } & \text { Affiliation } & \text { Email } \\ \text { Corwin, W. } & \text { DOE } & \text { william.corwin@nuclear.energy.gov } \\ \text { Grandy, C. } & \text { ANL } & \text { cgrandy@anl.gov } \\ \text { Hill, R.N. } & \text { ANL } & \text { bobhill@anl.gov } \\ \text { Li, M. } & \text { ANL } & \text { mli@anl.gov } \\ \text { Messner, M.C. } & \text { ANL } & \text { messner@anl.gov } \\ \text { Natesan, K. } & \text { ANL } & \text { natesan@anl.gov } \\ \text { Phan, V.T. } & \text { ANL } & \text { pvantung@anl.gov } \\ \text { Sham, T.-L. } & \text { ANL } & \text { ssham@anl.gov } \\ \text { Sofu, T. } & \text { ANL } & \text { tsofu@anl.gov } \\ \text { Vilim, R.B. } & \text { ANL } & \text { rvilim@anl.gov } \\ \text { Wang, Y. } & \text { ORNL } & \text { wangy3@ornl.gov } \\ \text { Wright, R.N. } & \text { INL } & \text { richard.wright@inl.gov }\end{array}$






\section{Argonne}

\section{Nuclear Engineering Division}

Argonne National Laboratory

9700 South Cass Avenue, Bldg. 208

Argonne, IL 60439

www.anl.gov 